University of Tennessee Health Science Center

UTHSC Digital Commons

$12-2016$

\title{
A Subject Specific Surface Electromyography Model For Estimating L4/L5 Compressive Loading
}

\author{
Pablo Joaquin Dopico \\ University of Tennessee Health Science Center
}

Follow this and additional works at: https://dc.uthsc.edu/dissertations

Part of the Other Analytical, Diagnostic and Therapeutic Techniques and Equipment Commons, and the Therapeutics Commons

\section{Recommended Citation}

Dopico, Pablo Joaquin (http://orcid.org/0000-0001-9325-6854), "A Subject Specific Surface Electromyography Model For Estimating L4/L5 Compressive Loading" (2016). Theses and Dissertations (ETD). Paper 414. http://dx.doi.org/10.21007/etd.cghs.2016.0420.

This Thesis is brought to you for free and open access by the College of Graduate Health Sciences at UTHSC Digital Commons. It has been accepted for inclusion in Theses and Dissertations (ETD) by an authorized administrator of UTHSC Digital Commons. For more information, please contact jwelch30@uthsc.edu. 


\title{
A Subject Specific Surface Electromyography Model For Estimating L4/L5 Compressive Loading
}

\author{
Abstract \\ Introduction: Low Back Pain (LBP) is a leading cause of activity limitation worldwide and results in 149 \\ million lost work days annually in the United States. The total costs of LBP are estimated to be $\$ 100$ to \\ $\$ 200$ billion annually. Specific causes of LBP account for less than $15 \%$ of LBP cases. Heavy loading of \\ the lumbar spine and tight hamstrings have been identified as risk factors for LBP. Tight hamstrings pull \\ the pelvis posteriorly and reduce the lordosis of the spine, resulting in increased loading of the lumbar \\ vertebral bodies of the spine. While this mechanism has been described, a link between hamstring \\ stretching and reduced lumbar vertebral body loading has not been established. Development of an \\ objective method to measure lumbar loading would assist is determining a link between tight hamstrings \\ and lumbar loading. Electromyography (EMG) modeling is a non-invasive tool shown to report \\ physiologically appropriate lumbar loading. In this thesis I developed a non-invasive EMG model to \\ measure L4/5 compressive loading and applied the model to measure differences in $L 4 / 5$ compressive \\ loading before and after a six-week hamstring stretching program. Background: Instrumented Vertebral \\ Body Replacements (VBR), as well as Biomechanical and EMG modeling have all been previously reported \\ as methods to determine spinal lumbar loads. VBR is useful for measuring loads in vivo, but cannot be \\ used for healthy subject populations. Biomechanical modeling using inverse dynamics has been shown \\ to report physiologically accurate values, but may not match underlying muscle activity. \\ Electromyography modeling reports physiological compressive loading, but currently makes use of a \\ single gain value relating muscle force to EMG muscle activity, assuming a linear relationship for all \\ subjects. Muscle force to EMG relationships previously revealed non-linear relationships and high percent \\ error values for general relationships. In this thesis, I attempted to develop a patient specific EMG model \\ for measuring L4/L5 compressive loading. I hypothesize that use of a subject specific muscle force to \\ EMG relationships will reduce percent error of muscle force estimation. Methods: Ten volunteer subjects \\ with tight hamstrings and no history of back injury were recruited. EMG's were placed on six major trunk \\ muscles. Subjects completed 3 isokinetic voluntary contraction trials (VCT) at 30 and $60 \% \mathrm{sec}$. After \\ addition of 53 reflective markers on key anatomical landmarks, subjects performed a series of tasks \\ including straight leg stoop lifting (SLSL). All testing was repeated after a six week stretching protocol. \\ VCT trials and EMG data was used to build subject specific and general relationships for each muscle. \\ Subject specific relationships were used to calculate compressive lumbar loading during SLSL trials. \\ Compressive loading calculated using an AnyBody lower body model and from three subjects from VBR \\ provided by Orthoload were used for comparing models. Compressive loading values before and after the \\ six week stretching protocol were also compared by both AnyBody and the EMG model to determine the \\ effects of hamstring stretching. Results: Subject specific relationships showed a decreased percent error \\ in estimating muscle forces compared to general relationships. The EMG model appeared to vary in \\ loading estimations more than both Orthoload and AnyBody, though most subjects fell within an \\ appropriate range. Same day intra-subject variability testing showed a small difference in impulse \\ measurements between testing sessions. The AnyBody lower body model consistently reported lower \\ loading than both Orthoload and the EMG model. Neither the AnyBody model nor the EMG model found \\ any differences in compressive loading when comparing pre and post hamstring stretching. Discussion: \\ Our model is an initial step in the development of a patient specific EMG model. Inclusion of additional \\ muscles and accounting for antagonist muscle activity during VCT trials would improve the accuracy of \\ our model and are potential next steps. Lack of differences in pre and post hamstring stretching implies \\ that total compressive loading across the L4/L5 disk pre and post hamstring stretching does not change, \\ though it's possible the distribution of the load on the disc changes.
}




\section{Document Type}

Thesis

\section{Degree Name}

Master of Science (MS)

\section{Program}

Biomedical Engineering

\section{Research Advisor}

William M. Mihalko, PhD.

\section{Keywords}

Backpain, Electromyography, hamstrings, loading, lumbar modeling

\section{Subject Categories}

Analytical, Diagnostic and Therapeutic Techniques and Equipment | Medicine and Health Sciences | Other Analytical, Diagnostic and Therapeutic Techniques and Equipment | Therapeutics 


\title{
A Subject Specific Surface Electromyography Model for Estimating L4/L5 \\ Compressive Loading
}

\author{
A Thesis \\ Presented for \\ The Graduate Studies Council \\ The University of Tennessee \\ Health Science Center
}

\begin{abstract}
In Partial Fulfillment
Of the Requirements for the Degree

Master of Science

In the Joint Graduate Program in Biomedical Engineering and Imaging

From The University of Tennessee

And

The University of Memphis
\end{abstract}

By

Pablo Joaquin Dopico

December 2016 
Copyright (C 2016 by Pablo Joaquin Dopico. All rights reserved. 


\section{DEDICATION}

This thesis is dedicated to my parents, Alejandro Dopico and Maria Dopico, no words can fully describe how thankful I am for such supportive parents. 


\section{ACKNOWLEDGEMENTS}

I would like to acknowledge my advisor, Dr. William Mihalko, for giving me the opportunity and guidance to complete my thesis. Dr. Audrey Zucker-Levin, for her time testing subjects with us and providing valuable advice. Dr. Brooke Sanford, for providing invaluable feedback towards the development of my model. Dr. Kunal Singhal, for helping me work through difficult complications in model development. My fellow graduate student, Michael Braman, for helping with early stages of model development, testing assistance, and tolerating my many brainstorming sessions. 


\begin{abstract}
Introduction: Low Back Pain (LBP) is a leading cause of activity limitation worldwide and results in 149 million lost work days annually in the United States. The total costs of LBP are estimated to be $\$ 100$ to $\$ 200$ billion annually. Specific causes of LBP account for less than $15 \%$ of LBP cases. Heavy loading of the lumbar spine and tight hamstrings have been identified as risk factors for LBP. Tight hamstrings pull the pelvis posteriorly and reduce the lordosis of the spine, resulting in increased loading of the lumbar vertebral bodies of the spine. While this mechanism has been described, a link between hamstring stretching and reduced lumbar vertebral body loading has not been established. Development of an objective method to measure lumbar loading would assist is determining a link between tight hamstrings and lumbar loading. Electromyography (EMG) modeling is a non-invasive tool shown to report physiologically appropriate lumbar loading. In this thesis I developed a non-invasive EMG model to measure L4/5 compressive loading and applied the model to measure differences in L4/5 compressive loading before and after a six-week hamstring stretching program.
\end{abstract}

Background: Instrumented Vertebral Body Replacements (VBR), as well as Biomechanical and EMG modeling have all been previously reported as methods to determine spinal lumbar loads. VBR is useful for measuring loads in vivo, but cannot be used for healthy subject populations. Biomechanical modeling using inverse dynamics has been shown to report physiologically accurate values, but may not match underlying muscle activity. Electromyography modeling reports physiological compressive loading, but currently makes use of a single gain value relating muscle force to EMG muscle activity, assuming a linear relationship for all subjects. Muscle force to EMG relationships previously revealed non-linear relationships and high percent error values for general relationships. In this thesis, I attempted to develop a patient specific EMG model for measuring L4/L5 compressive loading. I hypothesize that use of a subject specific muscle force to EMG relationships will reduce percent error of muscle force estimation.

Methods: Ten volunteer subjects with tight hamstrings and no history of back injury were recruited. EMG's were placed on six major trunk muscles. Subjects completed 3 isokinetic voluntary contraction trials (VCT) at 30 and $60^{\circ} / \mathrm{sec}$. After addition of 53 reflective markers on key anatomical landmarks, subjects performed a series of tasks including straight leg stoop lifting (SLSL). All testing was repeated after a six week stretching protocol. VCT trials and EMG data was used to build subject specific and general relationships for each muscle. Subject specific relationships were used to calculate compressive lumbar loading during SLSL trials. Compressive loading calculated using an AnyBody lower body model and from three subjects from VBR provided by Orthoload were used for comparing models. Compressive loading values before and after the six week stretching protocol were also compared by both AnyBody and the EMG model to determine the effects of hamstring stretching. 
Results: Subject specific relationships showed a decreased percent error in estimating muscle forces compared to general relationships. The EMG model appeared to vary in loading estimations more than both Orthoload and AnyBody, though most subjects fell within an appropriate range. Same day intra-subject variability testing showed a small difference in impulse measurements between testing sessions. The AnyBody lower body model consistently reported lower loading than both Orthoload and the EMG model. Neither the AnyBody model nor the EMG model found any differences in compressive loading when comparing pre and post hamstring stretching.

Discussion: Our model is an initial step in the development of a patient specific EMG model. Inclusion of additional muscles and accounting for antagonist muscle activity during VCT trials would improve the accuracy of our model and are potential next steps. Lack of differences in pre and post hamstring stretching implies that total compressive loading across the L4/L5 disk pre and post hamstring stretching does not change, though it's possible the distribution of the load on the disc changes. 


\section{TABLE OF CONTENTS}

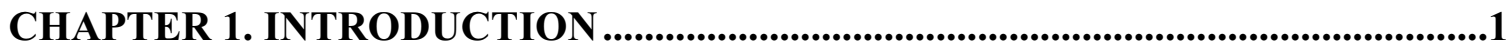

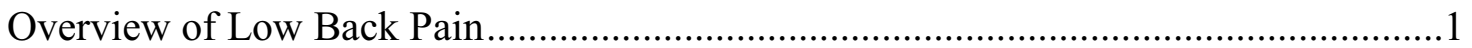

Anatomical Planes ...........................................................................................2

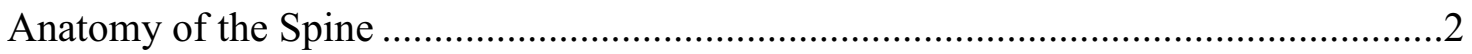

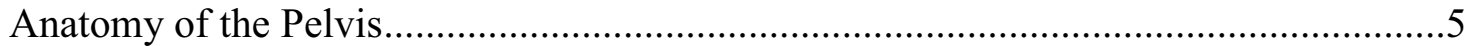

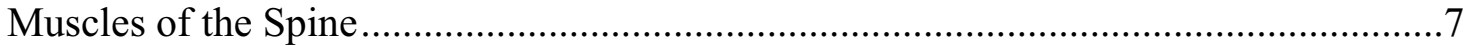

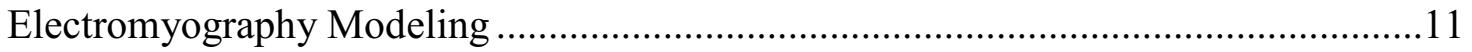

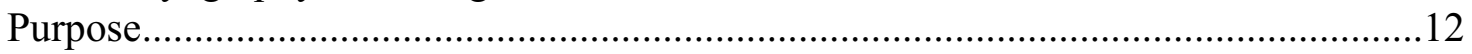

CHAPTER 2. MEASURING LUMBAR LOADING ........................................13

Vertebral Body Replacements ............................................................................... 13

Biomechanical Modeling to Estimate Spine Loading .................................................13

Electromyography Modeling to Estimate Spine Loading.........................................16

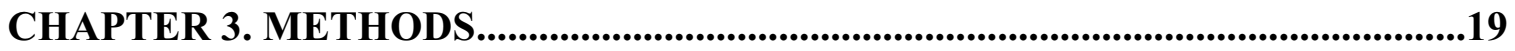

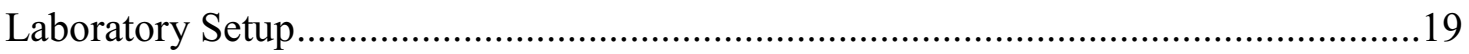

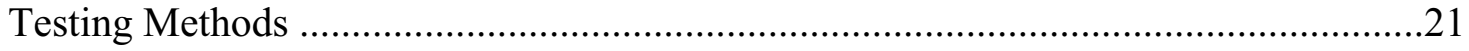

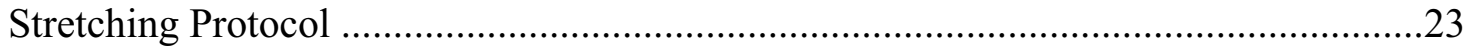

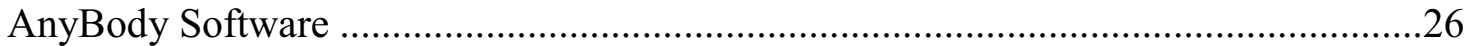

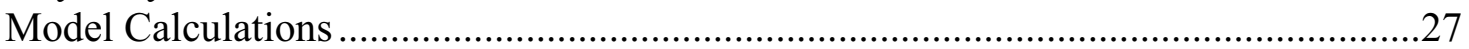

Building a Force to Surface Electromyography Relationship ..............................27

Estimating Compressive Loading from Surface EMG Activity .............................36

Orthoload Measurements of Compressive Lumbar Loading .....................................36

Data Analysis and Statistical Methods ................................................................... 37

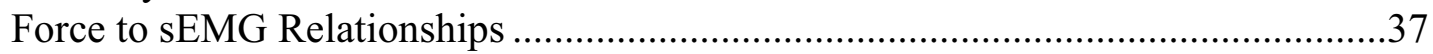

Comparison between Anybody, and EMG Model ............................................... 37

Pre and Post Lumbar Loading of Straight Leg Stoop Lifting ..................................38

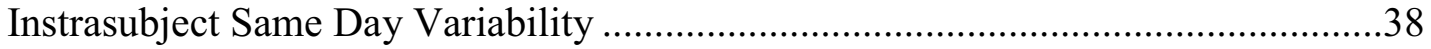

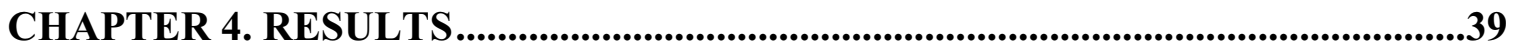

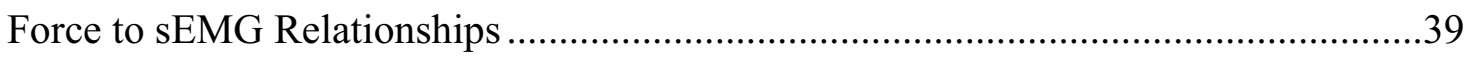

Comparison between EMG Model, AnyBody, and Orthoload ...................................39

Pre and Post Lumbar Loading of Straight Leg Stoop Lifting ....................................42

Instrasubject Same Day Variability ............................................................... 42

CHAPTER 5. DISCUSSION ...........................................................................................43

LIST OF REFERENCES ....................................................................................46

APPENDIX A. INSTITUTIONAL REVIEW BOARD.....................................................50

APPENDIX B. MATLAB CODE ....................................................................................52 


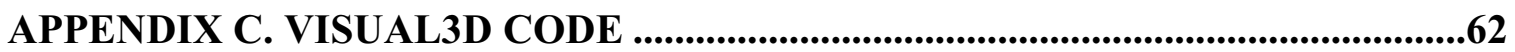

APPENDIX D. STATISTICAL RESULTS .............................................................

VITA 


\section{LIST OF TABLES}

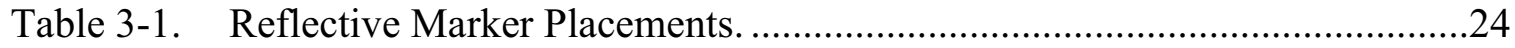

Table 4-1. Summary of Significance for Relationships. ............................................40

Table 4-2. Summary of Percent Error Values Divided by Measure Factor. .................40

Table 4-3. Summary of Paired t-Tests of Pre and Post Hamstring Stretch Loading......41

Table D-1. Full Multiple Measure ANOVA Results for the REO ...............................77

Table D-2. Full Multiple Measure ANOVA Results for the LEO..............................78

Table D-3. Full Multiple Measure ANOVA Results for the RRA................................79

Table D-4. Full Multiple Measure ANOVA Results for the LRA................................80

Table D-5. Full Multiple Measure ANOVA Results for the RES. ................................81

Table D-6. Full Multiple Measure ANOVA Results for the LES..................................82 


\section{LIST OF FIGURES}

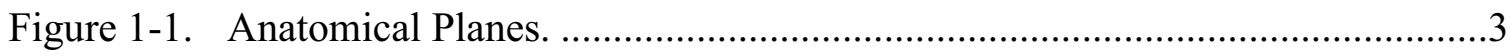

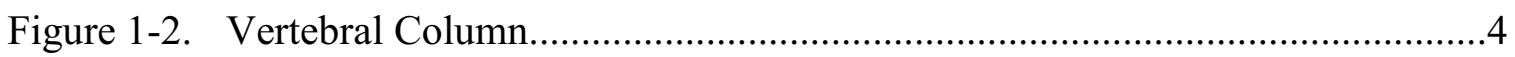

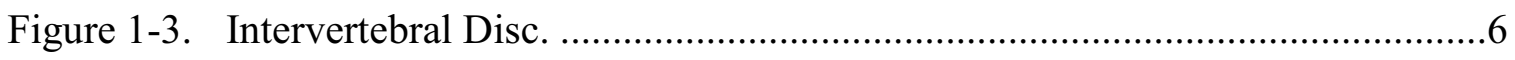

Figure 1-4. Pelvis Anatomy ............................................................................6

Figure 1-5. Effect of Tight Hamstrings on Lordosis. ....................................................

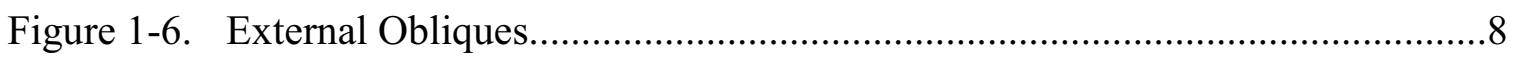

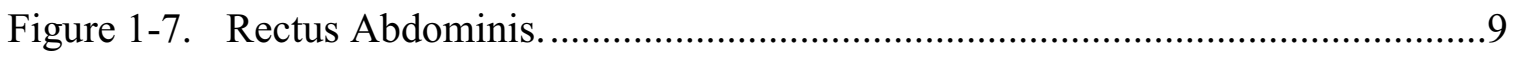

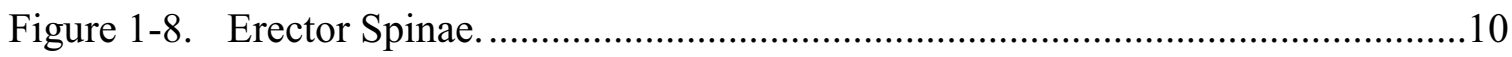

Figure 2-1. Synex Spine Vertebral Disk Replacement.....................................................14

Figure 2-2. Inverse Dynamics Example. ....................................................................14

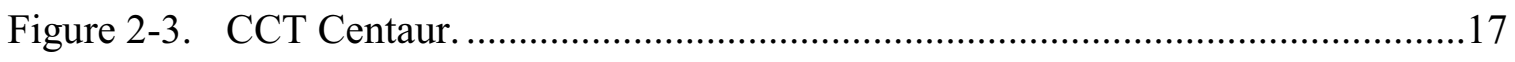

Figure 2-4. Isometric Contraction Apparatus. ............................................................. 17

Figure 3-1. Laboratory Coordinate Frame ………………………............................20

Figure 3-2. Passive Knee Extension Test. ..................................................................22

Figure 3-3. Initial Dynamometer Setup...............................................................22

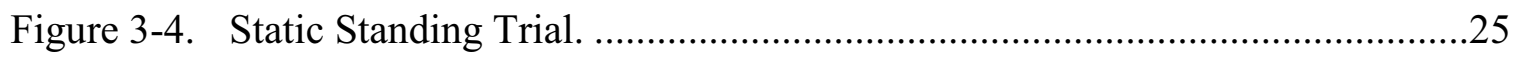

Figure 3-5. Linear Muscle Recruitment......................................................................28

Figure 3-6. Polynomial Muscle Recruitment............................................................29

Figure 3-7. AnyBody Lower Body Main Model............................................................30

Figure 3-8. Contribution of Torso Weight on Dynamometer Torque. .............................32

Figure 3-9. Muscle Force to sEMG Relationship for the Right Rectus Abdominus.......35

Figure 3-10. General Force to sEMG Relationship for the Left Erector Spinae. ...............35

Figure 4-1. Lumbar Loading of All Three Models...........................................................41 


\section{LIST OF ABBREVIATIONS}

\begin{tabular}{|c|c|}
\hline $\mathrm{AF}$ & Annulus Fibrosis \\
\hline AKE & Active Knee Extension \\
\hline CLBP & Chronic Low Back Pain \\
\hline DDD & Degenerative Disk Disease \\
\hline EAC & EMGworks Acquisition \\
\hline IVD & Intervertebral Disk \\
\hline LBP & Low Back Pain \\
\hline LEO & Left External Oblique \\
\hline LES & Left Erector Spinae \\
\hline LL & Lumbar Lordosis \\
\hline LRA & Left Rectus Abdominus \\
\hline NP & Nucleus Pulposus \\
\hline QTM & Qualysis Track Manager \\
\hline REO & Right External Oblique \\
\hline RES & Right Erector Spinae \\
\hline RRA & Right Rectus Abdominus \\
\hline SEMG & Surface Electromyography \\
\hline SLSL & Straight Leg Stoop Lifting \\
\hline VBR & Vertebral Body Replacement \\
\hline VCT & Voluntary Contraction Trials \\
\hline
\end{tabular}




\section{CHAPTER 1. INTRODUCTION}

\section{Overview of Low Back Pain}

Low Back Pain (LBP) is not a disease but rather a description of painful symptoms experienced by 70 to $85 \%$ of people within their lifetime [1-5]. Of these individuals, $85 \%$ will suffer recurrence $[3,4]$. While most cases of LBP are acute[1], at least 5 to $10 \%$ of individuals suffering from LBP will develop Chronic Low Back Pain (CLBP) [2]. Chronic Low Back Pain (CLBP) is a condition in which pain persists for a period of three or more months, though definitions may vary [2]. Regardless of acute or chronic, LBP frequently results in functional limitation and reduced mobility due to pain [3].

The high prevalence of LBP has resulted in significant socio-economic costs. LBP is the most common reason for medical consultations worldwide [1], activity limitation in individuals under the age of 45 [4], and results in the loss of 149 million workdays every year within the United States [6]. Lost work days and healthcare costs have resulted in total cost estimates ranging from $\$ 100$ to $\$ 200$ billion a year in the United States [6].

Multiple causes for LBP exist, both specific and non-specific. LBP is defined as specific when an underlying pathology is the known source of the pain. Specific causes of LBP include vertebral fractures and tumors, muscle spasms, and degenerative disk disease (DDD) [1]. DDD is the most common specific cause of CLBP and has been linked to increased loading on the Intervertebral Disk (IVD) $[5,7,8]$. Non-specific is often acute and accounts for $85 \%$ of patients suffering from LBP [9]. LBP is defined as non-specific when no anatomical pathology can be identified as the source of pain $[1,9]$.

While underlying anatomical pathologies are difficult to identify as the cause of LBP, multiple risk factors are associated with LBP. Both large and cumulative loading of the spine has been linked to increased incidence of DDD and LBP [7, 8]. Increased body mass index (BMI) [10], frequent lifting of heavy loads [11], and increased periods of sitting [12] all display an increase in LBP incidence due to resulting increased IVD loading [13]. Another risk factor common to individuals with LBP is decreased hamstring extensibility, though a link between hamstring extensibility and IVD loading has not been established [14].

Treatments for LBP include spinal fusions, intervertebral body replacements (VBR), epidural injections, medication, and physical therapy. In serious cases of stenosis or DDD, or when a fracture is present in a vertebral body, spinal fusion or insertion of a VBR can be done to relieve pain $[15,16]$. A spinal fusion involves the addition of a bone graft between two vertebral bodies, removing movement between the bodies and reducing LBP [15]. While spinal fusion is effective at reducing LBP, it commonly results in degeneration of adjacent IVD leading into recurrence of LBP $[15,17]$.

Similar to spinal fusion, VBRs are used to stabilize the spine. This is done by 
surgically replacing an anterior vertebral body and the IVD that is the source of pain with a VBR $[16,18]$. Most VBRs are commonly made of a combination of titanium, due to its osteointegration properties, ceramics, due to their strong wear properties, and carbon [16, $18,19]$. While both spinal fusion and VBR are capable of reducing LBP, they are the last treatments chosen for reducing LBP due to the high level of invasiveness involved in both procedures [20].

Medication, including oral, topical, or epidural injections for the treatment of LBP is often used as a source of short term relief and is often done in conjunction with physical therapy to reduce CLBP $[21,22]$. Physical therapy is commonly prescribed to alleviate LBP and has shown both short and long term success [23]. Multiple treatment methods exist including massage, back strengthening, and stretching, including hamstring lengthening. Hamstring lengthening reduces posterior pelvic tilt and increases Lumbar Lordosis (LL) [24]. Changes in Lumbar Lordosis (LL) can result in lower compressive loading on the vertebral bodies in lumbar spine, and therefore reduced LBP [25].

Currently, there is a lack of an objective method to find a relationship between hamstring stretching and reduced loading on the IVD. The development of a method to measure compressive loading would result in a better measure of the efficacy of hamstring stretching and other physical therapy treatments in reducing LBP.

\section{Anatomical Planes}

In order to understand the anatomy of the spine and its motion, the anatomical planes and directions must be defined within context of the human body. There are three major anatomical planes shown in Figure 1-1: the sagittal plane, the coronal plane, and the transverse plane. The sagittal plane travels vertically through the human body, dividing it into left and right halves. The coronal plane travels vertically through the midline of the human body, but divides it into a front and back portion. Finally, the transverse plane travels horizontally through the human body, dividing the body into a top and bottom portion [26].

\section{Anatomy of the Spine}

The human spine is a bony structure normally composed of twenty-four small bones called vertebrae $[13,26]$. Each adjacent pair of vertebrae and the intervertebral disc (IVD) between them makes up a motion segment [13]. The spine is responsible for protecting the spinal cord and transferring load between the pelvis and trunk, while allowing for motion of the trunk [13]. The spine can be divided into the cervical, thoracic spine, and lumbar spine [13]. Of these, the lumbar spine is most important since it is the source of LBP (Figure 1-2) [4, 9].

The lumbar spine is the inferior section of the spine between the thoracic and sacral regions and is curved posteriorly, a condition known as lordosis. 


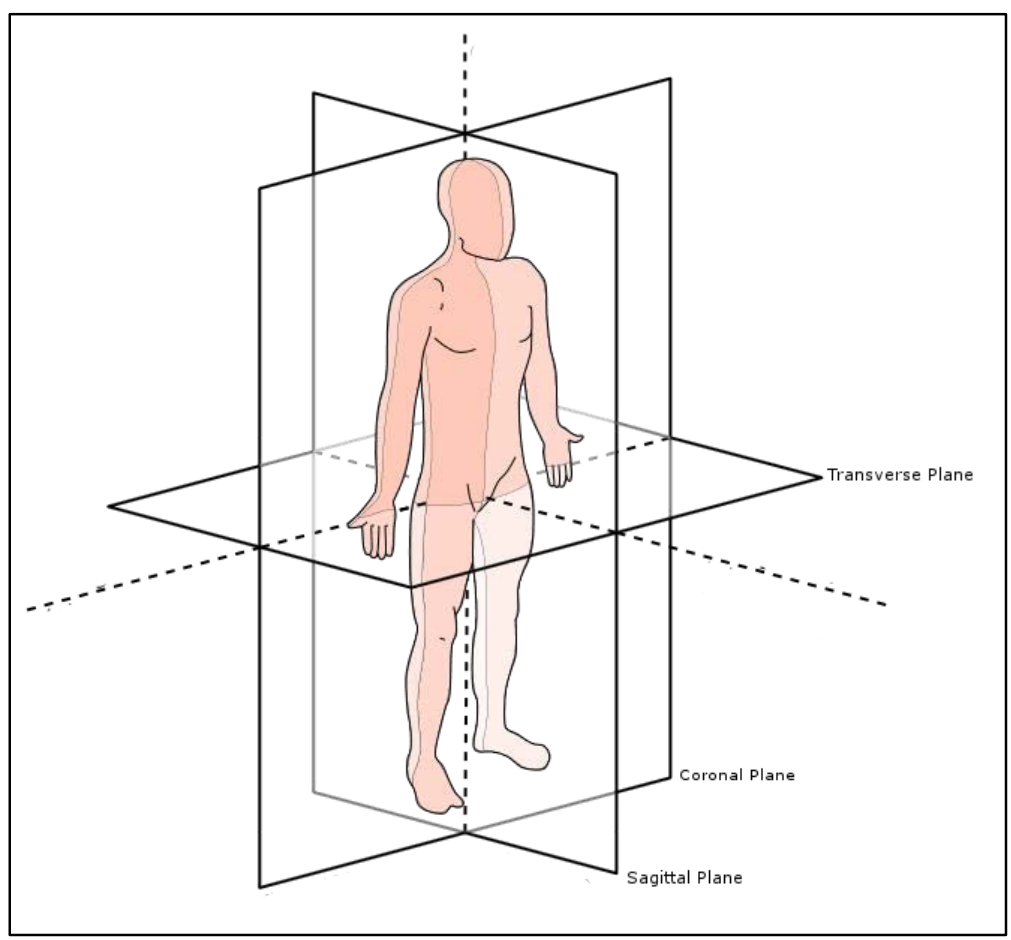

Figure 1-1. Anatomical Planes.

The transverse, sagittal, and coronal planes are the three anatomical planes of the human body.

Modified with permission. Edoarado. 2011 [cited 2016 May 30]; Available from:

https://commons.wikimedia.org/wiki/File:Anatomical_Planes-en.svg

Accessed 5/30/2016 [27] 


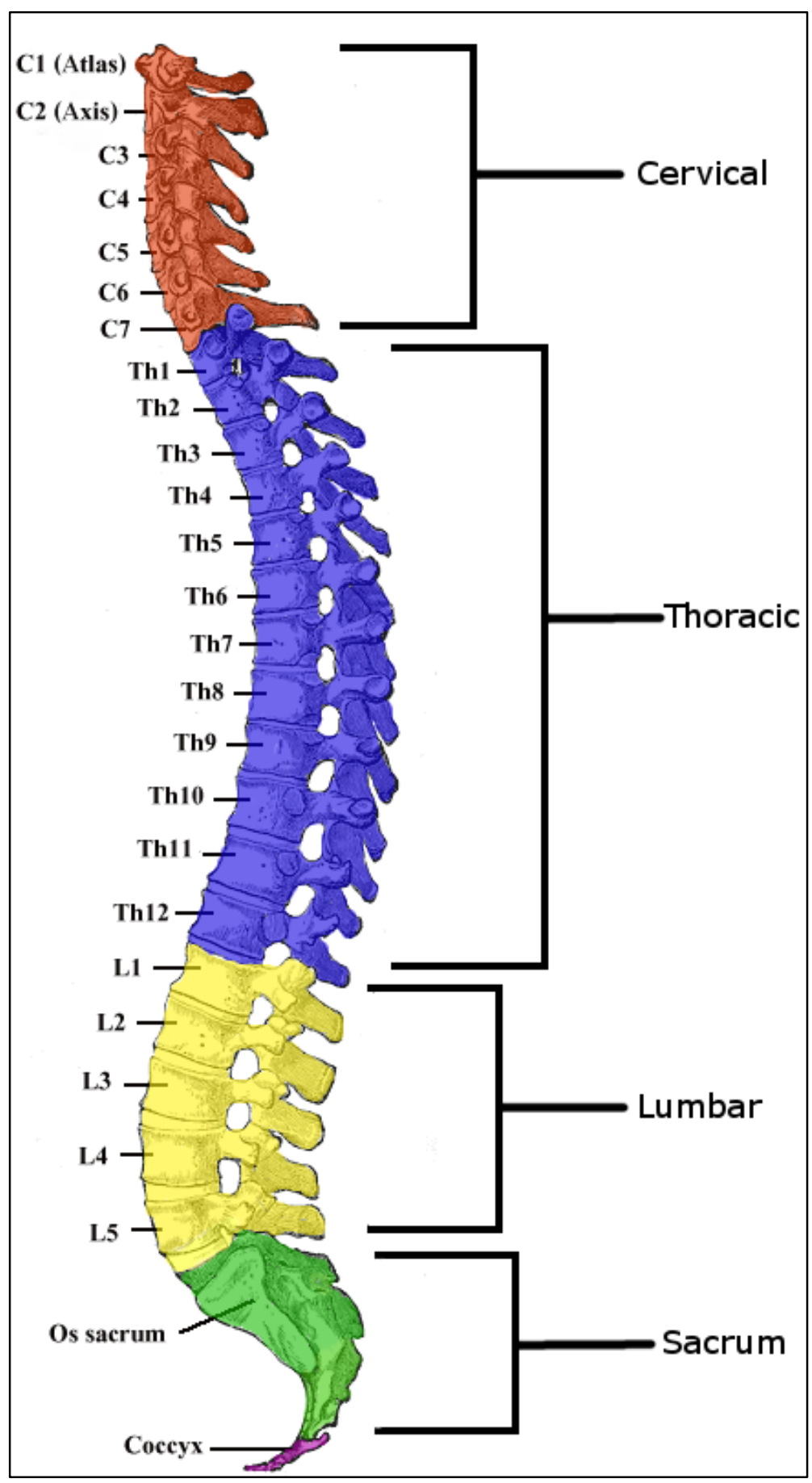

Figure 1-2. Vertebral Column.

The vertebral column is divided into the cervical (red), thoracic (blue), and lumbar (yellow) spines. The lumbar spine attaches to the sacrum (green).

Reprinted with permission. Gray, H., Anatomy of the Human Body. 20 ed, ed. W.H. Lewis. 1918, Philadelphia and New York: Lea and Febiger. [28] 
The lumbar spine is the inferior section of the spine between the thoracic and sacral regions and is curved posteriorly, a condition known as lordosis. It is normally composed of five vertebral bodies (L1 to L5) [26], though a sixth vertebral body (L6) is found in about $4 \%-6 \%$ of the population $[29,30]$. This additional vertebral body usually has no effect on spinal health, but may lead to incorrect vertebral segment identification [29]. IVDs are named by adjacent vertebra, the L4/L5 IVD, for example, lies between the L4 and L5 vertebra. The lumbar spine allows for flexion and extension of the torso and rotation where it meets the sacrum at the lumbosacral joint. The lumbar spine is responsible for supporting the weight of the entire torso and as such, the lumbar vertebrae are larger than the cervical and thoracic vertebrae $[13,26]$. The last lumbar motion segment (L4-L5) is subject to greater loading than the rest of the spine, which results in the intervertebral disk in between them being more prone to degeneration $[31,32]$. As a result, the lumbar spine is the most common source of LBP [26].

While vertebrae differ depending on which region of the spine they lie in, most vertebrae share common features, shown in Figure 1-3. Each vertebrae consists of an anterior cylindrical body that bears $80 \%$ of spinal loading and a posterior vertebral arch [33].The vertebral arch is made up of pedicles, small projections from the body, which connects to lamina, the posterior end of the vertebral arch. Projecting from the vertebral arch are the transverse processes, spinous processes, and facets. The facets of adjacent vertebrae connect to form a facet joint which bears $20 \%$ of spinal loading $[26,33]$. The amount of loading passing through the anterior body relative to the facet joints is altered by the lordosis of the spine [33].

The vertebral bodies of each motion segment are held together by an IVD, shown in Figure 1-3. The IVD of each motion segment serves to absorb and redistribute loads placed on the spine $[13,26]$. The two main sections of the IVD are the Nucleus Pulposus (NP) and the Annulus Fibrosis (AF) [5]. The AF is a tough fibrous ring primarily made up of layers of type 1 collagen fibers that surround the NP and forms the structure of the disk $[5,26]$. The NP commonly lies near the center of the IVD, though it is shifted posteriorly in lumbar IVD's [13]. Unlike the AF, the NP has a gel-like consistency. Within the NP are chondrocyte-like cells which produce type II collagen and a proteoglycan called aggrecan [5]. Aggrecan imbibes water and is responsible for the gellike fluidity of the NP [13].

As loads are placed on the IVD, the NP redistributes hydraulic pressure evenly throughout the disk while under a compressive load [13]. Under high compressive loading tears can form in the AF, which allows the NP to leak aggrecan and lose its ability to retain water. The resulting loss in disk height and stability causes spinal nerve impingement leading to LBP. This series of events is defined as DDD [34].

\section{Anatomy of the Pelvis}

The pelvis, shown in Figure 1-4, lies inferior to the lumbar spine and consists of three bones, the coccyx, sacrum and innominate bone. The sacrum is attached to the 


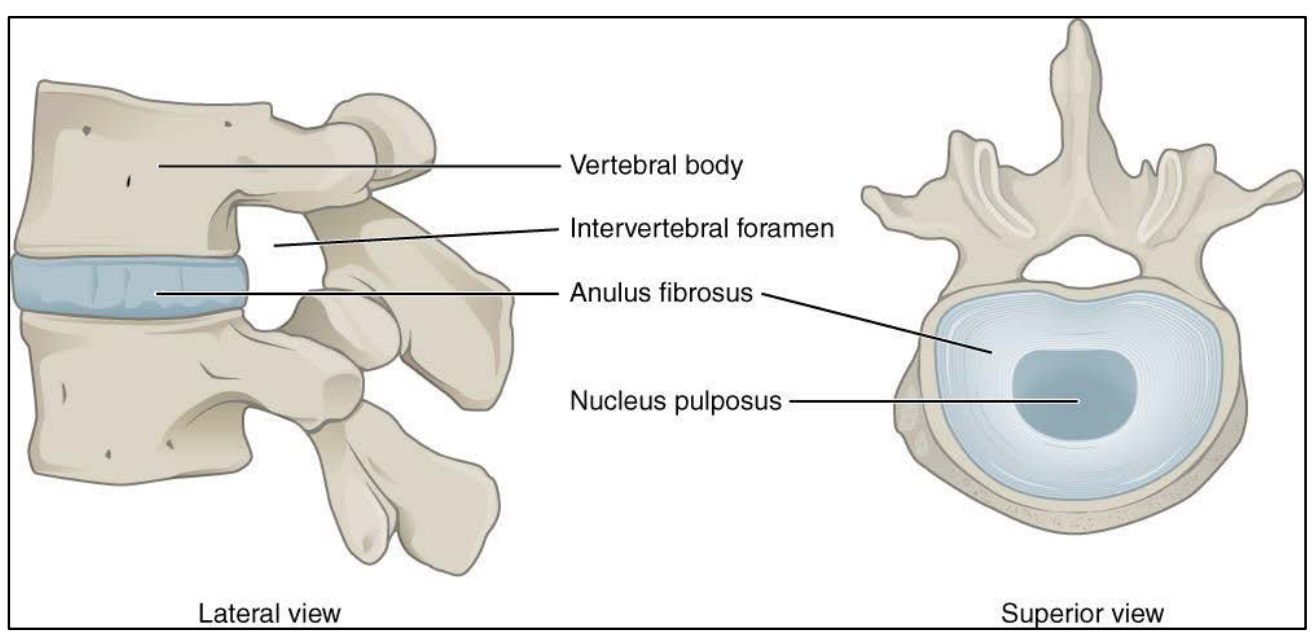

Figure 1-3. Intervertebral Disc.

The two vertebra and the disk in between (pictured left) is defined as a motion segment. Reprinted with permission. OpenStax. Anatomy \& Physiology. 2014 [cited 2016 May 5]; Available from: http://cnx.org/contents/14fb4ad7-39a1-4eee-ab6e-3ef2482e3e22@6.27 Accessed: 5/30/2014 [35]

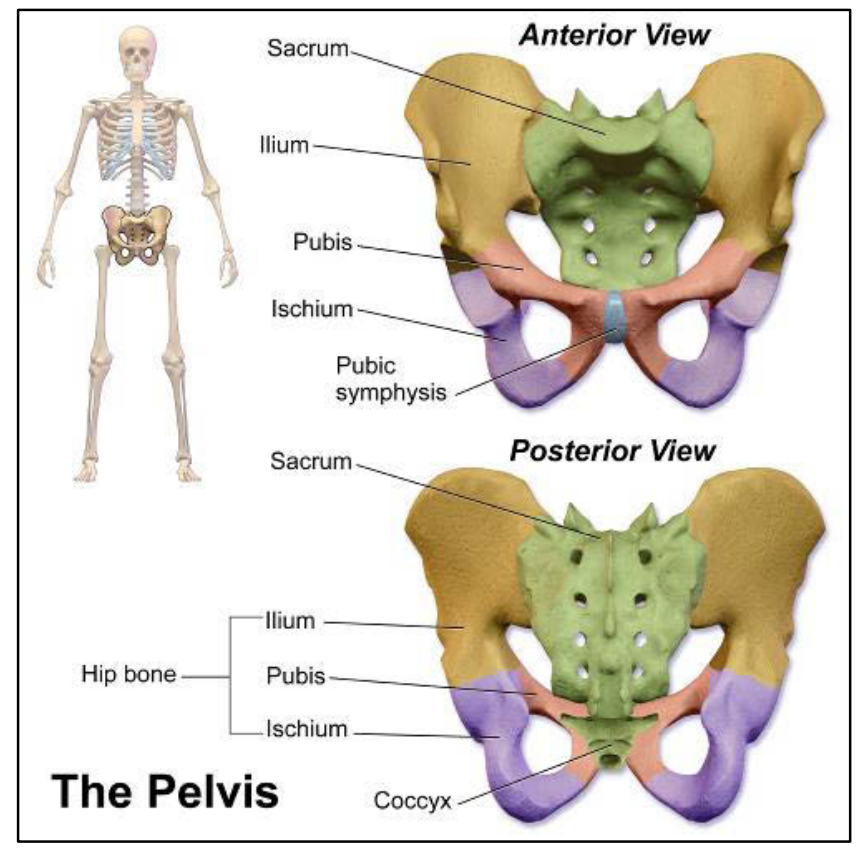

Figure 1-4. Pelvis Anatomy.

Reprinted with permission. BrusBlaus. Pelvis. Blausen Gallery 20142013 [cited 2016 May 30]; Available from:

https://en.wikiversity.org/wiki/Wikiversity_Journal_of_Medicine/Blausen_gallery_2014 Accessed: 5/30/2016 [36] 
lumbar spine and is one large bone formed from a fusion of five vertebrae. Inferiorly attached to the sacrum is the coccyx, commonly known as the tailbone. Finally, attached laterally to the sacrum is a pair of innominate bones formed from three bones that have fused early in development, the ilium, ischium, and pubis.

The ilium is the most superior portion of the hip and has a flat wing like appearance that extends laterally. The edge of the ilium, known as the iliac crest, commonly forms a visible shape on the skin. Both the anterior and posterior faces of the ilium are common attachment points for muscles. Inferior to the ilium is the ischium, which along with the ilium and pubis, forms the acetabulum where the femur inserts. Anterior to the ischium is the pubis. The superior edge of the pubis forms the pubic crest where the rectus abdominus inserts. Medially the pubis attaches to the second pubis of the second innominate bone via the symphysis pubis.

The hamstrings are a set of three muscles located posteriorly on the leg, the semitendinosus, the semimembranosus, and the biceps femoris. These muscles originate on the ischial tuberosity and attach to the tibia and the fibula, crossing both the hip and knee joint. These muscles are responsible for knee flexion and hip extension. When an individual has short or inextensible hamstrings, the hamstrings pull the pelvis posteriorly as shown in Figure 1-5.

\section{Muscles of the Spine}

The muscles responsible for movement of the spine include both extensors, which are generally attached directly to the spine, and the flexors, which attach to the pelvis, spine and ribs. Unlike muscles located in the limbs, muscles in the spine are generally a collection of muscles called fascicles [15]. Within context of this study, the main extensor muscle of the back is the Erector Spinae (ES) [15]. The major flexor muscles discussed in this study include the external obliques (EO) and rectus abdominis (RA) [15].

The external oblique's, shown in Figure 1-6, originate from the fifth through twelfth ribs and inserts into iliac crest, pubis, and linea alba. The linea alba is a fibrous structure primarily composed of connective tissue that travels from the sternum to the pubis. Along with the internal obliques and rectus abdominus, the external oblique's forms the abdominal wall which is primarily responsible for trunk movement and spinal flexion.

The rectus abdominus, shown in Figure 1-7, is a long flat muscle that originates on the sternum and fifth through seventh ribs and inserts into the pubic crest. Tendinous bands cross the rectus abdominus which forms the typical "6 pack" appearance present in muscular individuals. The rectus abdominus is important for posture, lumbar flexion, and posterior rotation of the pelvis.

The erector spinae (Figure 1-8) is the main extensor muscle of the back and also 


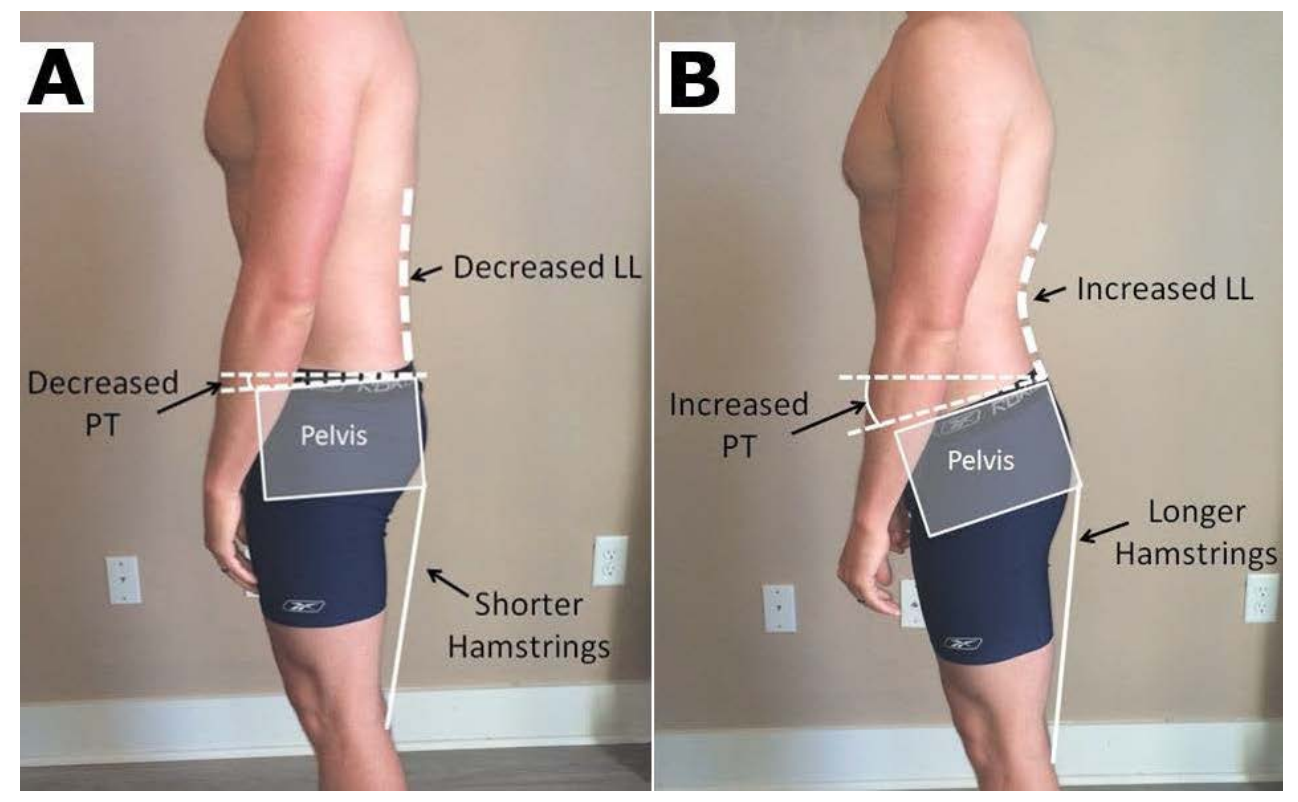

Figure 1-5. Effect of Tight Hamstrings on Lordosis.

Image produced by Michael Braman.

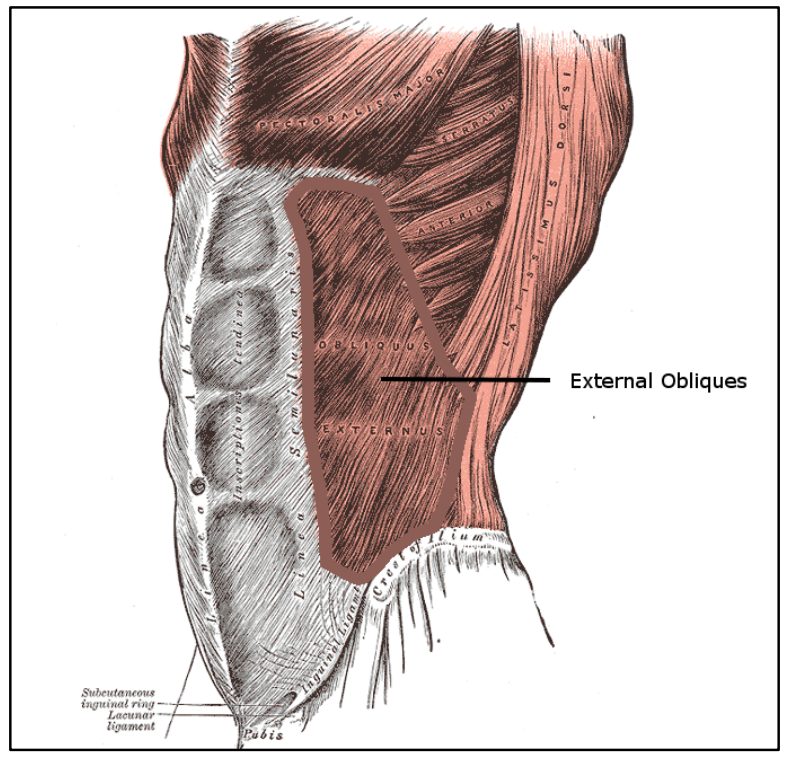

Figure 1-6. External Obliques.

Modified with permission. Gray, H., Anatomy of the Human Body. 20 ed, ed. W.H. Lewis. 1918, Philadelphia and New York: Lea and Febiger. [28] 


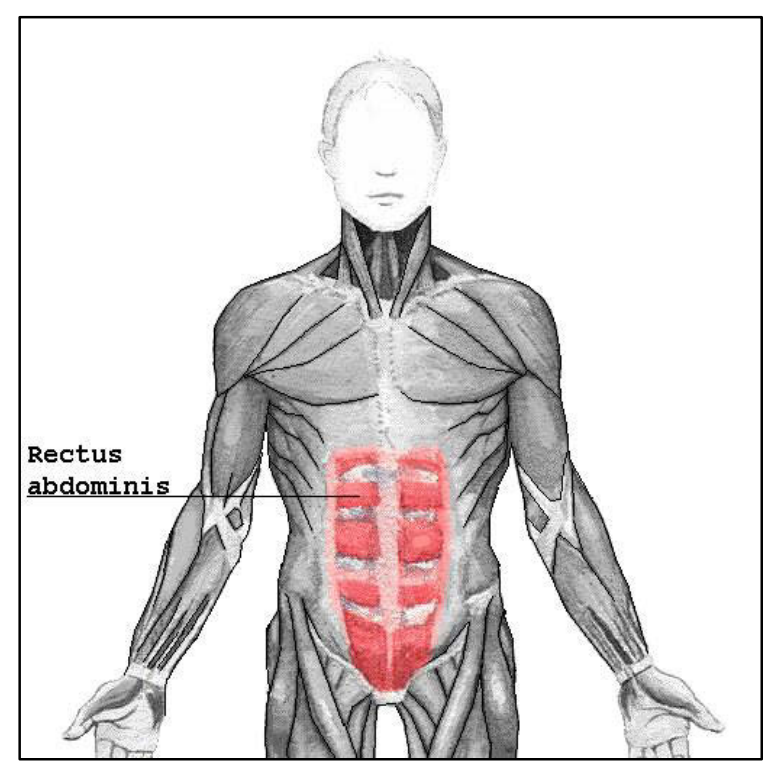

Figure 1-7. Rectus Abdominis.

Reprinted with permission. Rectus Abdominis. 2005 [cited 2016 May 30]; Available from: https://commons.wikimedia.org/wiki/File:Rectus abdominis.png

Accessed: 5/20/2016 [37] 


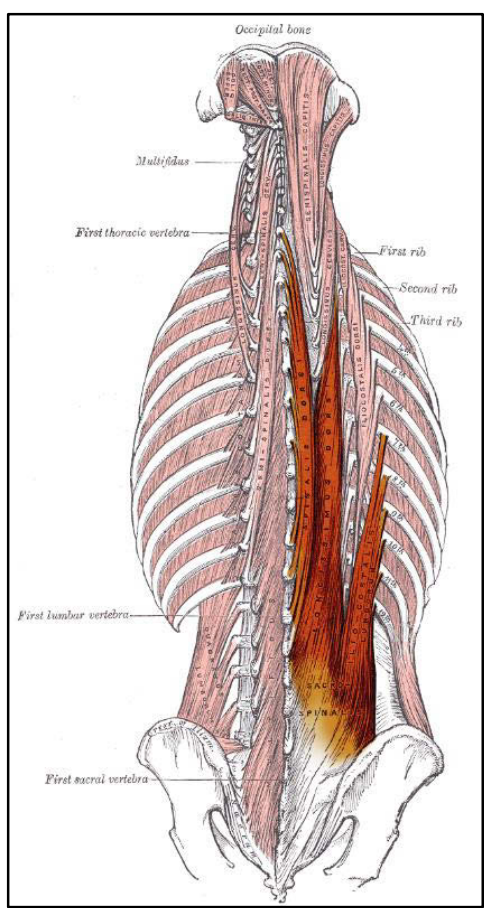

Figure 1-8. Erector Spinae.

The erector spinae (highlighted in red) consists of three major muscle groups. These are (from left to right) the spinalis, longissimus, and iliocostalis.

Reprinted with permission. Gray, H., Anatomy of the Human Body. 20 ed, ed. W.H. Lewis. 1918, Philadelphia and New York: Lea and Febiger. [28] 
the shallowest. It has multiple origins at the sacrum, iliac crest, and the spinous processes of the eleventh thoracic through fifth lumbar vertebrae. The ES is largest within the lumbar region before it divides into three different muscle masses with different insertions. The three muscles masses of the ES, from most to least medial, are the spinalis, longissimus, and illiocostalis. The iliocostalis inserts into the sixth through twelfth ribs and into $\mathrm{C} 4$ through $\mathrm{C} 6$. The longissimus inserts into transverse processes of the lumbar, thoracic, and cervical vertebrae. Finally, the spinalis inserts into the spinous processes of the lumbar, thoracic, and cervical vertebrae as well as the occipital protuberance on the skull. The ES, through its multiple branches, is responsible for spinal extension and lateral flexion. In many cases, weakness or spasms of the ES contributes to Low Back Pain (LBP).

\section{Electromyography Modeling}

EMG modeling is the recording of muscle activity by either surface or intramuscular electrodes. Surface EMG noninvasively measures muscle activity by measuring the collection of motor unit action potentials innervating a muscle. While EMG does not record muscle force, it is well known that EMG activity on non-fatigued muscles correlates to muscle force [38]. EMG modeling makes use of muscle forces crossing a joint to estimate compressive loading. Surface EMGs are unable to measure deep muscle activity. Additionally, muscle activity signals can vary greatly with BMI, electrode placement, muscle length during contraction, muscle physiological crosssectional area, and contraction velocity [38-40]. Muscle physiological cross-sectional area is the cross sectional area of a muscle perpendicular to the muscle fiber orientation and can often be estimated by dividing the volume of the muscle by the length of the muscle fibers [40]. Despite these limitations, EMG modeling has shown the ability to estimate L4/L5 compressive loading at physiologically appropriate values[38, 41].

Early EMG modeling on lumbar loading was done by Granata et al for lifting tasks. Granata calculated muscle forces for five bilateral trunk muscles which then had their vector components summed to estimate lumbar shear and compression. Like most EMG modeling, Granata made use of a gain value relating muscle force to EMG signals. Moments calculated from Granatas model showed a strong correlation $\left(\mathrm{R}^{2}>0.8\right)$ to lifting moments measured from load cells placed on the subject for over $80 \%$ of their lifting trials [38]. A similar model to the one used by Granata et al was compared with biomechanical and hybrid modeling in measuring compressive loads during sagittal plane lifting by Gagnon et al. All three models displayed differences in estimated muscle forces, though they all reported similar compressive loading values. Collectively these values were above physiological values [33, 39]. Another hybrid model by Castanharo et al made use of kinematic data and muscle activity during sitting postures to estimate L4/L5 compressive loading. Compressive values reported by Castanharo $(1279 \mathrm{~N} \pm 112)$ fell within previously reported ranges [33, 41]. 


\section{Purpose}

There is currently a lack of objective assessment of physical therapy treatment efficacy for LBP. While methods such as mathematical modeling and EMG modeling currently exist for assessing L4/L5 lumbar loading, these methods currently rely on general assumptions. Electromyography presents a noninvasive way to physiologically measure muscle activation to estimate lumbar loads. In this study I developed a patient specific EMG model for estimating L4/L5 lumbar loading. I hypothesize that use of our electromyography model will result in physiologically appropriate L4/L5 lumbar loading estimates, validated by comparison with in vivo lumbar loading measurements by instrumented IVD.

Additionally, I made use of my model, as well as a lower body AnyBody model, to asses L4/L5 lumbar loading differences during straight leg stoop lifting in individuals with tight hamstrings before and after a six-week hamstring stretching intervention. I hypothesize that hamstring stretching will result in reduced lumbar loading on the vertebral body. 


\section{CHAPTER 2. MEASURING LUMBAR LOADING}

Three major methods exist to measure compressive loading in the spine which includes mathematical modeling, electromyography (EMG) modeling, and surgical insertion of vertebral body replacement capable of measuring loads. Of these, vertebral body replacements (VBR) with instrumentation are the sole method of measuring in vivo loads on the lumbar spine. Orthoload (Berlin, Germany) has made use of VBR's to measure spinal loads in daily activities [16], weight carrying [42], and in relation to lumbar lordosis [25]. Mathematical modeling often makes use of movement and external load data in order to estimate forces acting on the joint. Estimations of joint forces are usually done by balancing moments around the joint. EMG modeling of joint loading is based off muscle activity surrounding the joint. EMG modeling is suitable for capturing physiological activity, but current methods are susceptible to substantial errors [43].

\section{Vertebral Body Replacements}

In cases where such as DDD, spinal stenosis, or compression fractures lead to significant instability in the spine, a Vertebral Body Replacement (VBR) can be surgically implanted to stabilize the spine $[16,18]$. These materials are commonly made out of titanium, due to its osteointegration properties, ceramics, due to their strong wear resistance, and carbon $[16,18,19]$. While multiple designs exist for VBR, the primary one used in replacements measuring lumbar loading is the Synex System (Synthes Spine, Oberdorf, Switzerland) shown in Figure 2-1 [16, 25, 42, 44].

Use of instrumented VBR allows for in vivo measurements of spinal loading. However, insertion of a VBR is extremely invasive as it involves surgery and the removal of unstable vertebral bodies, making it unsuited for studying patient populations that do not require a VBR. Additionally, there are some short comings of the instrumented Synex implant. Body temperature can vary within the body and while calibrations were done at three temperatures, there is a much greater continuum of body temperatures possible [46]. Additionally, the amount of loading points within the human body is likely higher than the amount of loading points used for calibration. Finally, the Synex implant is meant to replace the function of an anterior body and not an IVD, meaning that loading over the implant will likely not be fully identical to loading across the IVD.

\section{Biomechanical Modeling to Estimate Spine Loading}

Early biomechanical modeling was done by Schultz and Andersson. Their estimation of loads on the lumbar spine was calculated mathematically by balancing external moments and forces with internal muscle forces during static conditions [48]. A simplified illustration of this concept is provided in Figure 2-2.

In Schultz's model anthropomorphic data was used to estimate the weight and 


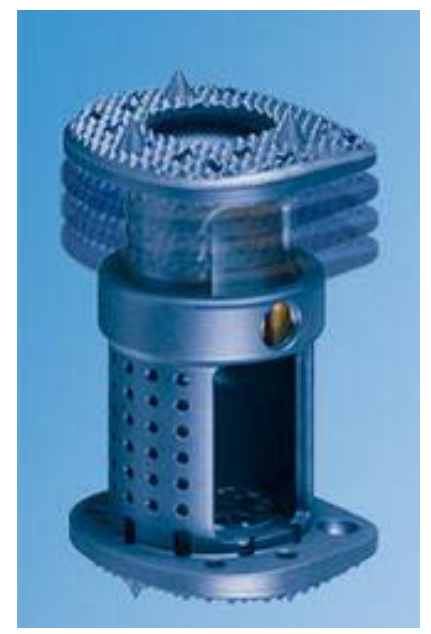

Figure 2-1. Synex Spine Vertebral Disk Replacement.

Reprinted with permission. Synex System. An expandable vertebral body replacement device. Technical Guide. 2001; Available from:

http://www.synthes.com/MediaBin/US\%20DATA/Product $\% 20$ Support $\% 20$ Materials/Tec hnique\%20Guides/SPTGSynexJ3704G.pdf

Accessed: 5/31/2016 [47]

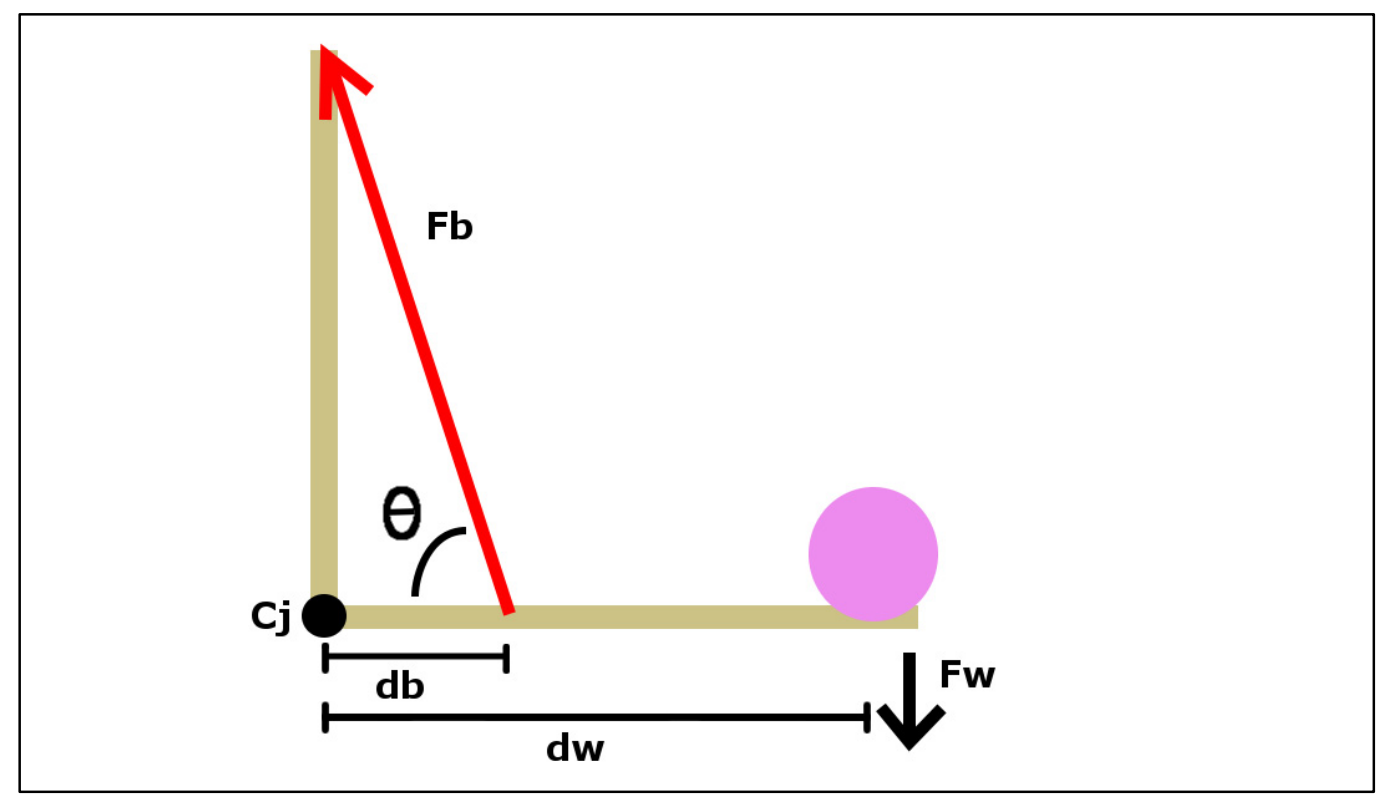

Figure 2-2. Inverse Dynamics Example.

In inverse dynamics internal moments are balance around a joint with known external moments. In this simplified example, the moment produced around $\mathrm{C}_{\mathrm{j}}$ by a known weight $\mathrm{F}_{\mathrm{w}}$ is the same as the moment produce by $\mathrm{F}_{\mathrm{b}}$. Inverse dynamic problems usually involve more than a single muscle. 
dimensions of the upper body. Upper body weight and dimensions, as well as any weight being held by the "subject" at the time of calculation, was used to calculate external moments and forces (called net moment and net force) around a vertebral segment. These external forces were then balanced by the compressive force on the spine and the internal muscle forces of ten muscles. These muscles bilaterally included the Latissimus Dorsi, External Obliques, Internal Obliques, and Erector Spinae. Since muscles forces are unknown and only six equations (three force directions and three moments) are available to estimate muscle forces and compression, assumptions were made about muscles forces. Antagonistic muscle activity was ignored and muscles forces were selected that minimized spine compression [48].

The model computed by Schultz and Anderrson strongly correlated with measures of myoelectric activity, intraabdominal pressures, and intradiscal pressures [48, 49]. SEMGs were placed three centimeters from the midline of the T8, L1, L2, and L5 vertebrae to pick up ES activity. For the flexor muscles, sEMGs were placed over the RA and EO muscles. Intradiscal pressures were measured by insertion of a needle containing a pressure transducer into the center of the third lumbar disc. To measure intra-abdominal pressure, subjects swallowed a radio transducer encapsulated in a polycarbonate cylinder. A metal diaphragm within the cylinder produced signals due to intra-abdominal pressure changes, which were then sent from the transducer and picked up by an antenna. Ten standing and fifteen sitting tasks were done by subjects to validate Schultz model [49].

Predicted compression and muscle forces correlated strongly with measured intradiscal pressures $\left(\mathrm{R}^{2}=.94\right)$ and ES sEMG activity respectively $\left(\mathrm{R}^{2}=.94\right)$. Flexor trunk muscles did not correlate very well and were particularly low for the right internal and external oblique muscle $\left(\mathrm{R}^{2}=.20\right)$. Both myoelectric activity and predicted compression values indicated that large moments placed on the spine resulted in larger compressive forces on the spine than large external forces, such as holding a weight close to the trunk [49].

Modern musculoskeletal modeling, such as AnyBody (AnyBody Technology, Aalbord, Denmark), makes use of similar methods in order to estimate joint loads. Inverse dynamic models make use of kinematic data and ground reaction force data to estimate internal muscle forces and joint reaction forces [50-52]. Unlike surface Electromyography modeling, Inverse dynamic modeling is capable of providing estimates on both superficial and deep muscles forces. Additionally, inverse dynamic modeling has been shown to produce physiologically appropriate loading values [51]. One major limitation of inverse dynamics is that it estimates joint torques produced by all muscles acting across the joint, because of this infinite solutions exist to balance moments around a joint $[52,53]$. In order to resolve this, assumptions must be made to determine how much each muscle contributes to joint torque [52]. Selection of muscles forces that minimize the torque on joints results in muscles with larger moment arms being favored [48], which may not reflect the actual underlying muscle activity [53]. 


\section{Electromyography Modeling to Estimate Spine Loading}

Surface Electromyography (sEMG) is a useful tool for noninvasively measuring muscle activity and has shown application for estimating compressive lumbar loading using muscle activity in trunk muscles at physiologically accurate values [38, 39]. These models often calculate muscle force by normalizing measured EMG signals and multiplying them by a gain value, establishing a linear relationship between muscle force and sEMG relationship, and correcting for muscle length, muscle physiological cross sectional area, and contraction velocity effects on muscle force [38, 39].

The relationship between muscle force and sEMG activity of trunk muscles is not fully understood. SEMG activity is known to vary with electrode placement and muscle strength [54]. Subject specific factors such as muscle size, muscle fatigue, and proportion of fast-twitch muscle fibers result in different muscle force production for different subjects [54-56]. These differences result in different muscle force to sEMG relationships for different subjects in the same muscle. SEMG and muscle force normalization partially resolves differences between individuals allowing for a general relationship to be constructed for all subjects.

Current studies on the relationship between muscle force and sEMG activity of trunk muscles report solely on isometric activity. In studies by Huebner at al, healthy subjects were placed into a CTT Centaur (BfMC, Germany) device, shown in Figure 2-3, meant to strengthen the back and improve posture. This device restricts lower body movement while tilting the subject's entire body, forcing the subject to stabilize their body against the force of gravity. SEMGs were placed on the RA, ES, EO, Internal Obliques, and Multifidus muscles. Subjects were asked to perform a Maximal Voluntary Contraction (MVC) trial by pushing against the harness of the CCT Centaur. Submaximal trials were gone by tilting subjects through a range of angles from $0^{\circ}$ to $90^{\circ}[57,58]$. Expected sEMG values were calculated by applying a sine function on the angle and normalizing by the MVC trials.

Huebner et al showed a non-linear relationship between muscle force and sEMG activity for abdominal muscles and a linear relationship for trunk extensors during isometric body tilt experiments $[57,58]$. Abdominal muscles showed lowered sEMG values than expected at low body tilts and higher values at higher tilts. The Multifidus showed higher values are low body tilts and the ES showed lower values at higher tilt. In the case of the ES these differences were substantial, indicating that using a single force to sEMG gain value to produce a trend line could result in significant overestimation in calculated and actual ES muscle forces [57].

Brown et al made an attempt to account for antagonist muscle activity during isometric tasks in a sitting position. In their study 14 sEMGs were placed on healthy males bilaterally on the RA, EO, Internal Obliques, Latissimus Dorsi, and three levels of the ES. Using a sitting apparatus described by Vera-Garcia et al and shown in Figure 2-4, subjects performed isometric contractions in four combinations: Extensor contraction with the torso upright, extensor contraction at $50^{\circ}$ of torso flexion, flexor contraction with 


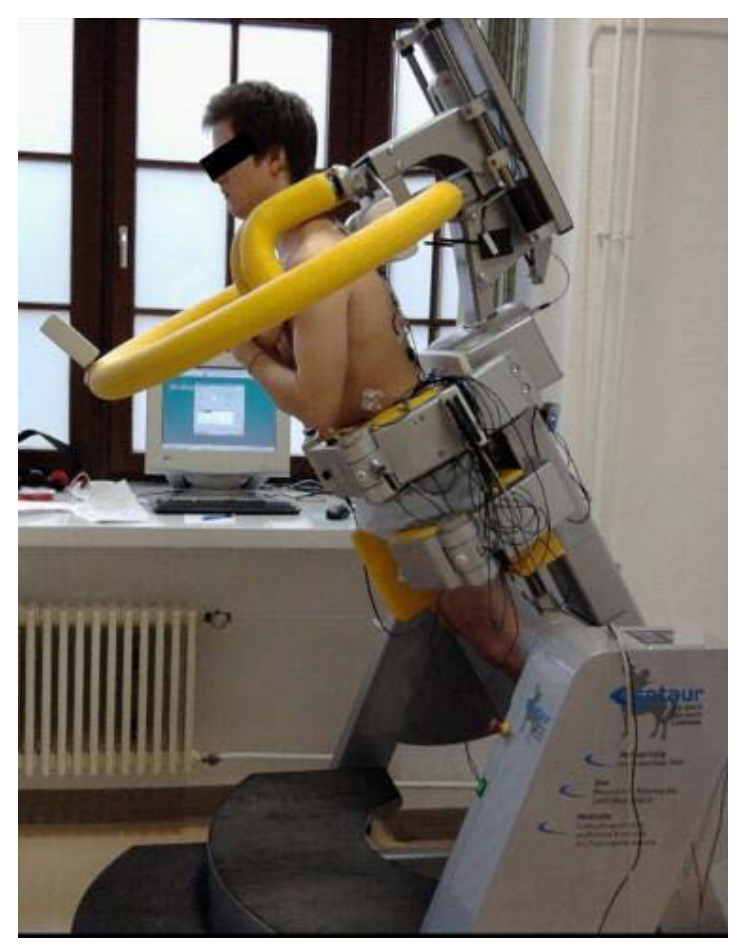

\section{Figure 2-3. CCT Centaur.}

Reprinted with permission. Brown, S.H.M. and S.M. McGill, Co-activation alters the linear versus non-linear impression of the EMG-torque relationship of trunk muscles. J Biomech, 2008. 41(3): p. 491-7 [43]

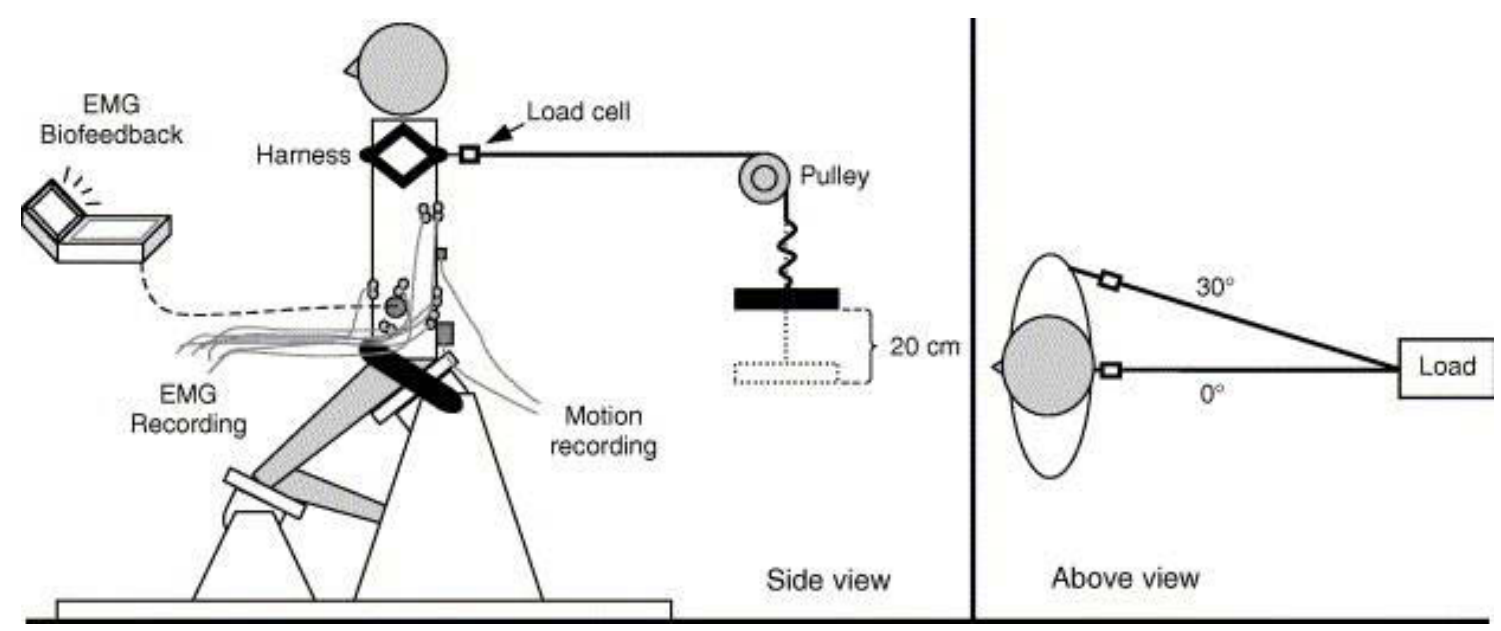

Figure 2-4. Isometric Contraction Apparatus.

Reprinted with permission. Vera-Garcia, F.J., et al., Effects of different levels of torso coactivation on trunk muscular and kinematic responses to posteriorly applied sudden loads. Clin Biomech (Bristol, Avon), 2006. 21(5): p. 443-55. [60] 
the torso upright, and flexor contraction at $50^{\circ}$ of torso flexion [43].

The muscle moment of agonist muscles was calculated both with and without accounting for antagonist muscle activity. In the case where antagonist muscle activity was ignored, the agonist muscle moment was equal to the external moment measured by the sitting apparatus [43]. To account for antagonist muscle activity individual muscle forces were calculated using a model described by McGill and Norman [43, 59]. The agonist muscle moment was then calculated as the sum of antagonist and external moments. Moments calculated for all individuals were pooled and plotted together to form a general relationship of agonist muscle moments versus normalized sEMG [43].

Brown et al observed that the flexor agonist moment to sEMG curve changed from a curved to linear relationship once antagonist muscle activity was accounted for. Additionally, extensor moment became slightly curved from linear once antagonist muscle activity was accounted for. General relationships formed by Brown et al showed that normalized sEMG values can still vary by as much as $40 \%$ of maximum sEMG for the same normalized moment for different individuals [43]. Suggesting that a general relationship or single gain value for all subjects may not satisfactory for estimating muscle forces using sEMG activity.

Huebner et al and Brown et al's force to sEMG studies involved isometric contractions. However, since muscle force activity varies with both fiber length and contraction velocity $[61,62]$, these isometric relationships may not be applicable to activities involving lumbar flexion and extension which are more similar to isokinetic activities. Changes in muscle forces without a concurrent change in sEMG activity could alter the linearity and relationship strength of muscle force to sEMG at different isokinetic speeds, resulting in incorrect muscle force estimates based on isometric or incorrect isokinetic contraction velocity muscle force to sEMG relationships.

In this thesis I tried to develop a patient-specific sEMG model for measuring lumbar loading during isokinetic activity. I am primarily interested in sEMG due to its non-invasiveness relative to IVD replacements and ability to more accurately describe underlying muscle activity relative to biomechanical modeling. I used both AnyBody and intervertebral disk replacement data to validate our sEMG model. I attempted to quantify differences in relationship strength for subject specific and general population muscle force to sEMG relationships. I hypothesized that subject specific muscle force to sEMG relationships of trunk muscles will produce significantly stronger relationships than a general normalized relationship. Additionally, I wanted to distinguish relationship linearity and strength differences at isokinetic activity at $30 \% \mathrm{~s}$ and $60 \%$ s. To the authors knowledge, there has not been a substantial effort to calculate muscle force to sEMG activity relationships for trunk muscles during isokinetic activity. I hypothesize that $60 \%$ isokinetic activity will produce lower muscle forces at equivalent sEMG activity than isokinetic activity at $30 \%$, but that these relationships will be non-linear for both $30 \% \mathrm{~s}$ and $60 \%$ s isokinetic activity. 


\section{CHAPTER 3. METHODS}

\section{Laboratory Setup}

All testing was done in the University of Tennessee Health Science Center (UTHSC) gait lab. Testing for this project made use of ten optoelectric cameras (Qualisys, Gothenburg, Sweden), three force plates (AMTI, Watertown, MA), an isokinetic dynamometer (Biodex, Shirley, NY), and the Trigno Wireless EMG system (Delsys, Natick, MA).

Oqus 110, 300, and 310 optoelectric cameras were used to collect kinematic data. Optoelectric cameras captured motion by releasing infrared light which reflected off reflective markers. Optoelectric cameras recorded at $100 \mathrm{~Hz}$ with a $640 \times 512$ resolution $(.034 \mathrm{~m} \times .042 \mathrm{~m}$ resolution at a maximum distance of $20 \mathrm{~m})$. Qualisys Track Manager (QTM) was used to simultaneously record marker positions and Electromyography (EMG) data. The coordinate frame of the lab was defined within QTM as shown in Figure 3-1. The $Y$ axis being defined as parallel to a path defined by the edge of a force plate, the $\mathrm{X}$ axis was perpendicular to the $\mathrm{Y}$ direction within the transverse plane, and the $\mathrm{Z}$ axis as vertical. The origin was defined at the corner of force plate one.

The Trigno Wireless EMG system was used to record EMG signals for muscles and to synchronize QTM and Biodex data. EMG data was recorded at $1000 \mathrm{~Hz}$. Baseline noise for EMG data was less than $<.5 \mathrm{mV}$ within Delsys Acquisition Software. Delsys Acquisition Software was used to synchronize QTM and Biodex, but QTM was ultimately used to record muscle activity. Rest trials showed an average error of $.01 \mathrm{mV}$ due to noise for EMG signals recorded within QTM.

AMTI OR4-5 Force plates, shown in purple in Figure 3-1, were used to track ground reaction forces during all trials. Force plates measured forces in all three directions (Fx, Fy, and Fz) via strain gage bridges. Maximum forces of $2224 \mathrm{~N}$ could be measured in the Fx and Fy direction, this value was doubled for the Fz direction. The resolution for Fx, Fy, and Fx was .725N/bit, .725N/bit, and 2.9N/bit respectively according to AMTI's output calculator.

The Biodex System 4 dynamometer can be used for isokinetic, isometric, or isotonic contractions. For the purpose of this thesis, the isokinetic features were primarily used. The System 4 dynamometer can maintain eccentric speeds of $300 \%$ s and concentric speeds of $500^{\circ} / \mathrm{s}$. Concentric and Eccentric torques of up to $680 \mathrm{Nm}$ and $544 \mathrm{Nm}$ respectively can be recorded via accompanying software. Biodex systems have been validated with external measures [63, 64]. A Trigno Analog Adapter was used to record Biodex data into an EMG channel. 


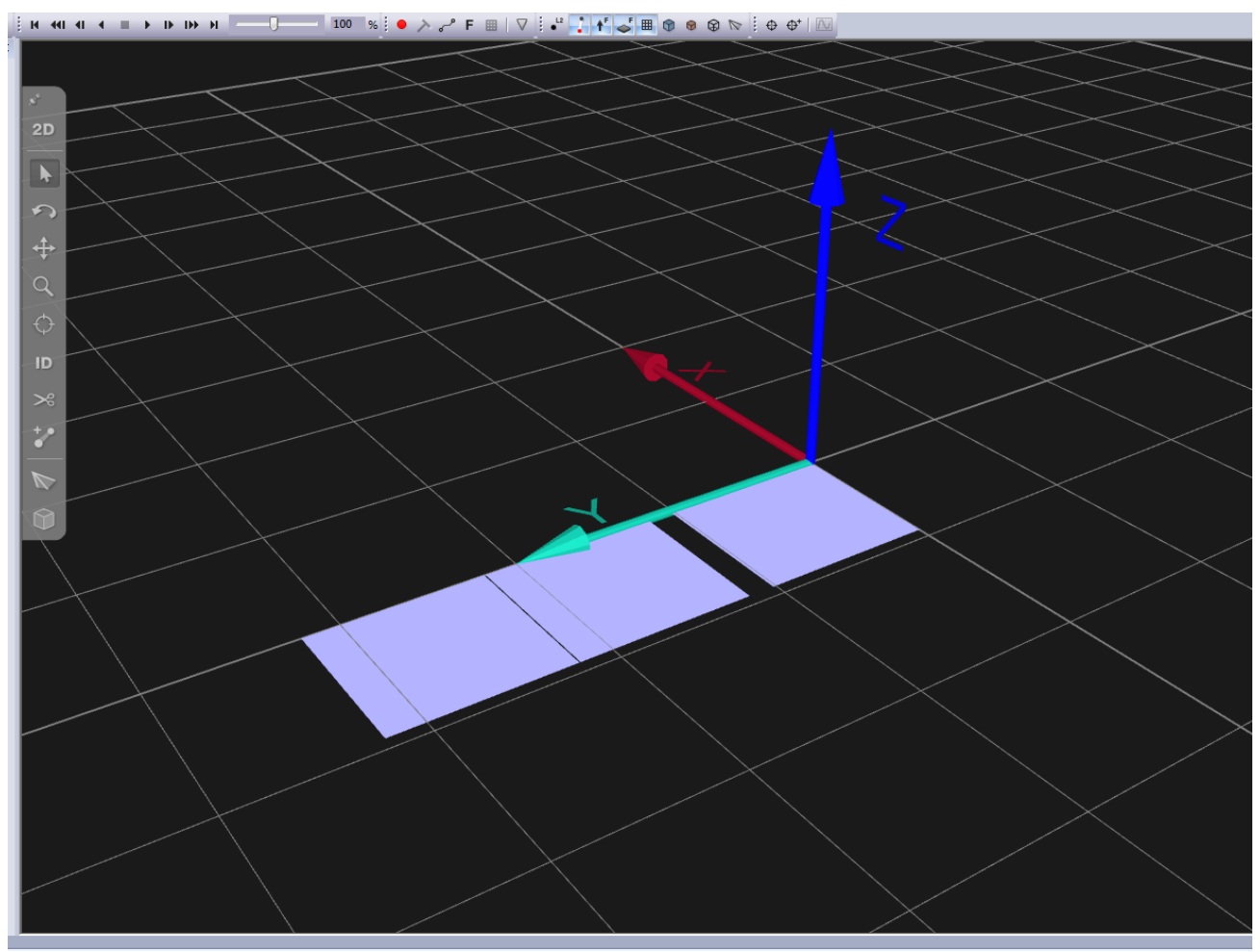

Figure 3-1. Laboratory Coordinate Frame. 


\section{Testing Methods}

IRB approval (shown in Appendix A) and informed consent was obtained from ten volunteer subjects $(25.2 \mathrm{y} \pm 3.2)$ with no history of LBP or injury. Hamstring length was assessed by a physical therapist using a Passive Knee Extension (PKE) test. A PKE test, shown in Figure 3-2, is done with the subject lying on their back with one limb passively moved to $90^{\circ}$ hip flexion. Each knee was independently, passively extended to reported discomfort and the angle between shank and vertical popliteal angle (PA) was measured. A PA greater than $25^{\circ}$ was required to participate in the study.

Surface Electromyography electrodes (sEMG's) were placed bilaterally on the Erector Spinae (ES), Rectus Abdominus (RA), and External Obliques (EO). Additional sEMG's were placed bilaterally on the Rectus Femoris, Biceps Femoris, Semitendinosis, and Gluteus Maximus. However, these sEMG's were part of a separate study and further discussion of their use is outside of the context of this thesis. One sEMG was reserved as a timestamp in order to synchronize Biodex data with sEMG data.

Once electrodes were properly placed, subjects performed two resting trials. Subjects were asked to lie on their back and relax with their eyes shut. After 30 seconds of relaxing, their resting sEMG activity was recorded twice for five seconds through Qualisys Track Manager (QTM). After completion of resting trials an examiner opened EMGworks Acquisition (EAC) in conjunction with QTM in preparation of the voluntary contraction trials.

To build a muscle force to sEMG relationship, a series of Voluntary Contraction Trials were done for each subject. Subjects were strapped on to a custom lever arm of the Biodex dynamometer while in a standing position as shown in Figure 3-3. This lever arm was a hip lever arm modified to work for spinal flexion and extension. A belt at the subject's hips was used to keep the subjects lower body in place and restrain their hips. The lever arm of the dynamometer was aligned so the axis of rotation of the lever arm aligned with the subject's sacrum. Zero degrees of rotation was defined as the subject standing vertically in the sagittal plane. Flexion resulted in an increase in the measured angle

Immediately before VCT's were done, a weight to body flexion angle curve was built for each subject. A scale was placed underneath the subject and the subject was asked to relax as much as possible at varying angles from a range of zero to sixty degrees of flexion. Both the torque measured by the lever arm and the weight recorded by the scale was recorded for future use. Once completed the scale was removed for the VCT's.

A series of isokinetic Voluntary Contraction Trials (VCT) were performed for each subject. Three sets of VCTs were done at 30 and 60 degrees of rotation per second throughout a range of 60 degrees of motion. During each set, subjects performed isokinetic flexion and extension three times. The first set of each speed was performed at maximal effort while being given verbal encouragement by the researchers. The second and third sets were performed at varying submaximal levels of effort. The subject was 


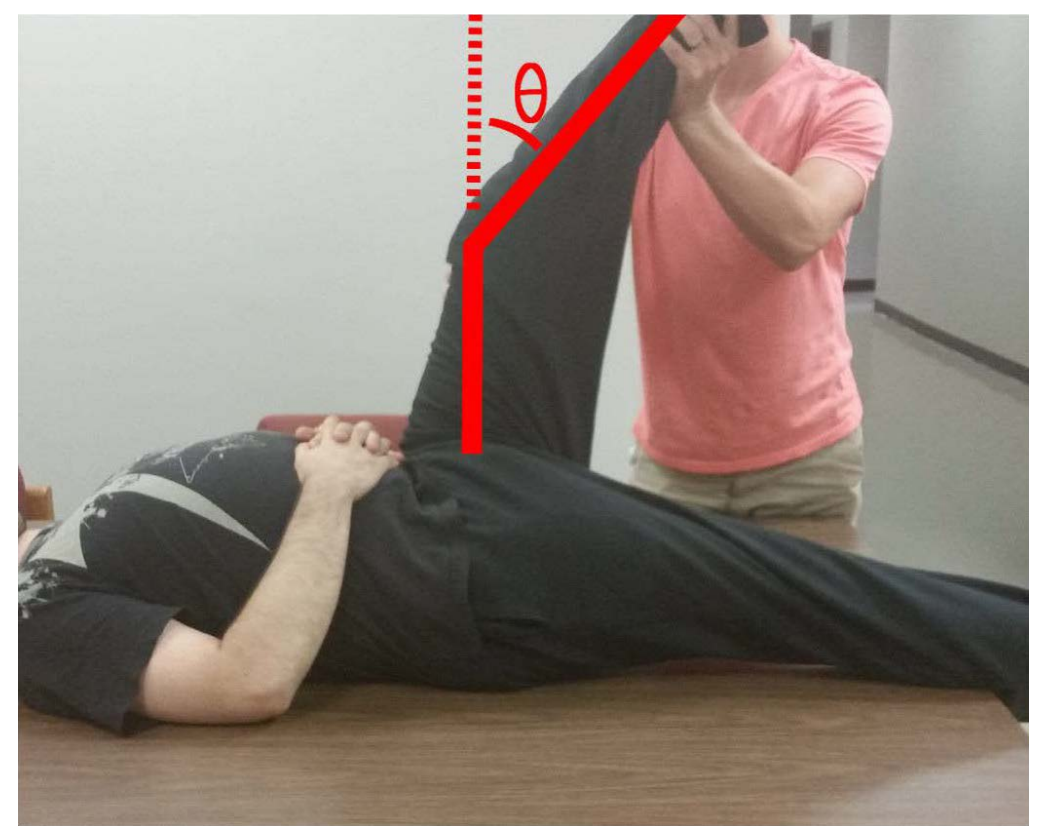

Figure 3-2. Passive Knee Extension Test.

In a PKE a physical therapist passively extends the subjects knee until they report a discomfort. A smaller $\theta$ indicates higher hamstring flexibility.

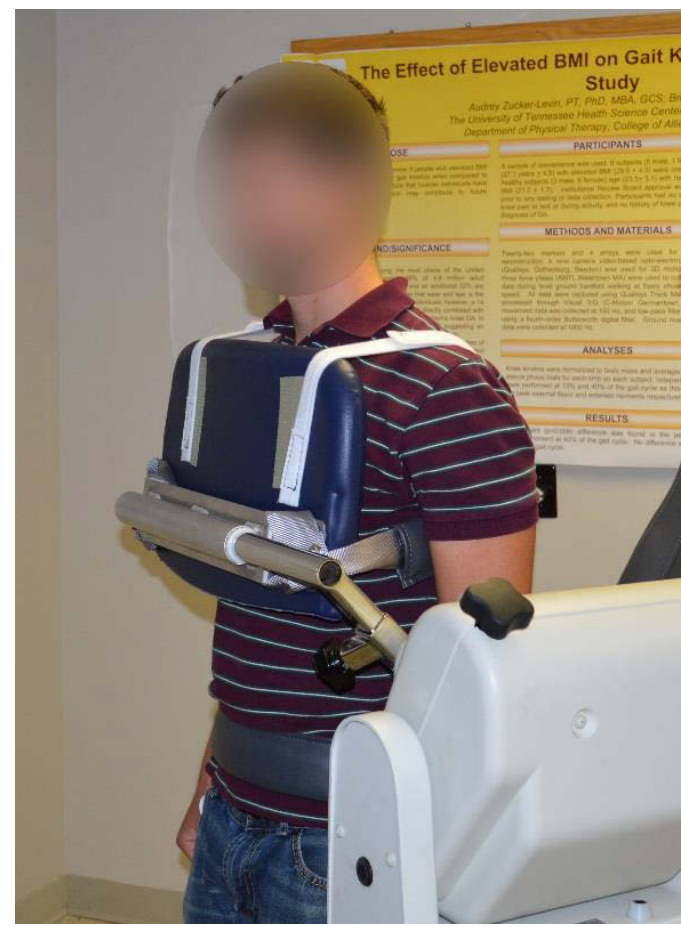

Figure 3-3. Initial Dynamometer Setup. 
instructed to provide 20,50 , and $80 \%$ effort on the first, second, and third repetition respectively for the second and third sets. Throughout each VCT, muscle activity measured by sEMG, torque measured by the Biodex Dynamometer, and the lever arm angle values were recorded concurrently. The submaximal trials served to better establish a force to sEMG signal relationship for each muscle.

Once VCT trials were completed EAC was closed as QTM alone was sufficient to record sEMG activity for experimental trials. 53 reflective markers were placed on anatomical landmarks of the torso and lower extremities. These markers were used to track each subject's kinematics as they performed everyday tasks in the gait lab. A full table of marker placements is provided below in Table 3-1.

Before any other trials were done, but after VCT's, subjects were asked to complete two static standing trials. Subjects stood, with both legs on a single force plate, rotated at about $45^{\circ}$ between the $\mathrm{X}$ and $\mathrm{Y}$ axis defined by the laboratory set up as shown in Figure 3-4. Subjects elevated their arms to about $45^{\circ}$ as they were recorded in standing posture for 5 seconds. Standing trials were later used to determine marker placement, subject width, depth, and leg length.

Subjects were asked to do a series of common tasks as part of a larger study. These tasks included walking, sit-to-stand, walking up and down stairs, toe touches into full lumbar extension, normal stoop lifting and straight leg stoop lifting (SLSL). This thesis and validation of the EMG model developed both primarily focused on SLSL.

An $8 \mathrm{~kg}$ medicine ball was used for SLSL lifting trials. A marker was placed on the ball to track its position throughout the trail. Subjects stood with one leg on each force plate and the medicine ball was placed one foot in front of the subject. Subjects were asked to reach down and lift the medicine ball, keeping their legs straight until they could not reach any further without bending their knees. Once lifted, subjects held the ball while standing up right for two seconds before setting it down once again while keeping their legs straight as much as possible. This process was repeated for a duration of thirty seconds. Straight leg lifting trials were done twice.

This testing session protocol was performed twice. After the first testing session subjects underwent a six week stretching intervention to improve their hamstring flexibility. After the six week stretching intervention, hamstring flexibility was reassessed and subjects underwent another testing session.

\section{Stretching Protocol}

A trained physical therapist directed and oversaw completion of each subjects stretching intervention. Each subject was required to meet with the physical therapist for a weekly supervised stretching session. Supervised stretching sessions served to ensure that subjects used proper form when stretching, track progress, and ensure compliance. After the first supervised stretching session, subjects were instructed to stretch on their 
Table 3-1. Reflective Marker Placements.

\begin{tabular}{|c|c|}
\hline Segment & Defined by markers on \\
\hline Torso & $\begin{array}{l}\text { Sternum } \\
\text { C7, T4, T7, T10, T12, L2, L4 Vertebral markers } \\
\text { Sacrum } \\
\text { Left and right Acromion }\end{array}$ \\
\hline Pelvis & $\begin{array}{l}\text { Left and right Posterior Superior Illiac Spine } \\
\text { Left and right Illiac Crest } \\
\text { Left and right Anterior Superior Illiac Spine }\end{array}$ \\
\hline Thigh (bilateral) & $\begin{array}{l}\text { Thigh plates containing four markers that form a square. Used } \\
\text { for rotation of the thigh. } \\
\text { Medial Femoral Condyle } \\
\text { Lateral Femoral Condyle }\end{array}$ \\
\hline Shank (bilateral) & $\begin{array}{l}\text { Tibial plates containing four markers that form a square. Used } \\
\text { for rotation of the tibia. } \\
\text { Medial Femoral Condyle } \\
\text { Lateral Femoral Condyle } \\
\text { Medial Malleolus } \\
\text { Lateral Malleolus }\end{array}$ \\
\hline Foot (bilateral) & $\begin{array}{l}\text { Medial Malleolus } \\
\text { Lateral Malleolus } \\
\text { Calcaneus } \\
1^{\text {st }} \text { and } 5^{\text {th }} \text { Metatarsal } \\
\text { Large Toe }\end{array}$ \\
\hline
\end{tabular}

Five rigid body segments were defined by markers on key bone landmarks. 


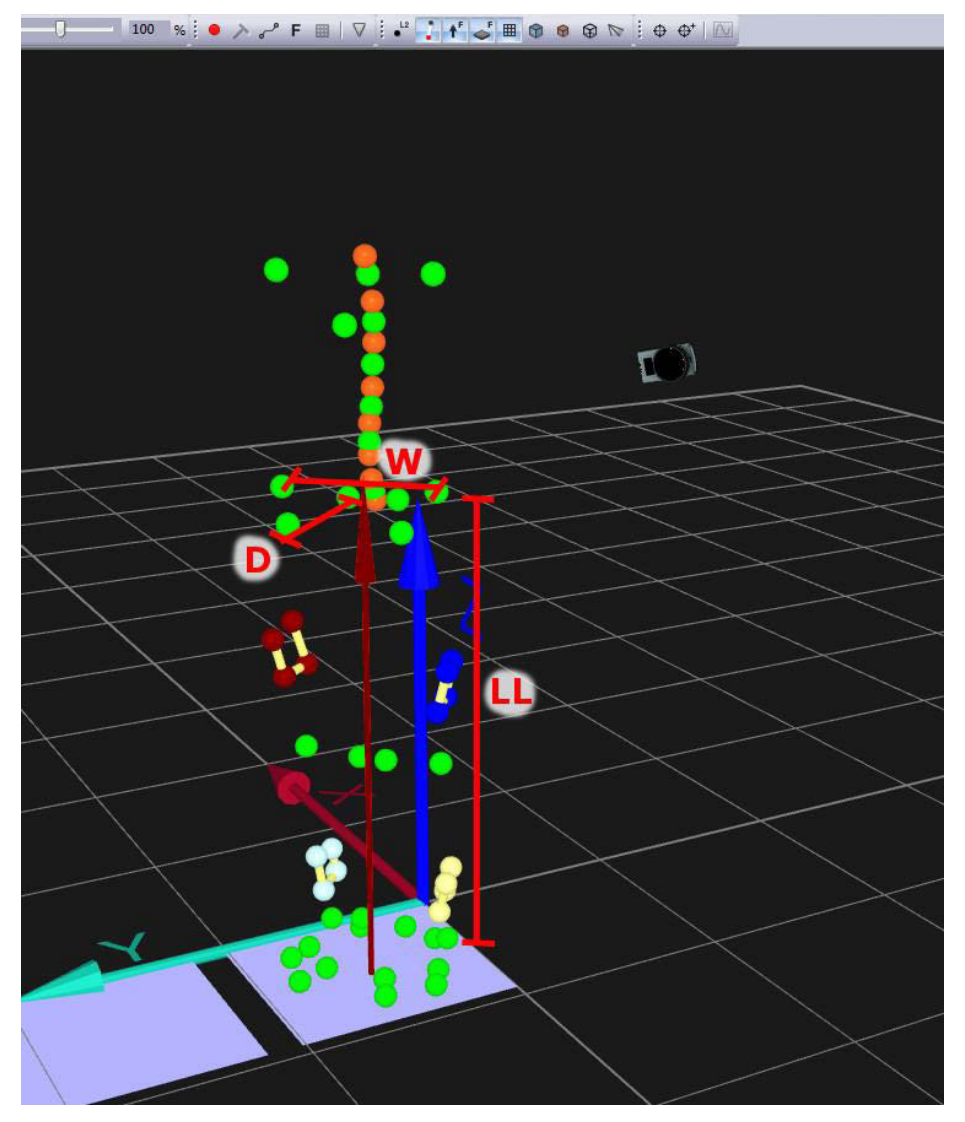

Figure 3-4. Static Standing Trial.

Image of a subject standing. Measures of subject width (W, calculated from both Left Illiac Crest and Right Illiac Crest), depth (D, calculated from Right Posterior Illiac Spine and Right Anterior Illiac Spine), and leg length (LL, calculated from Left Illiac Crest and Left Lateral Malleolus) are shown. 
own twice a day for three days of the week and maintain a compliance log.

Participants performed a supine static stretch described by DeCoster et al [65]. This stretch was selected due to its effectiveness in increasing hamstring flexibility [65] and its lack of specific instruction regarding pelvic position making it easier for subjects to perform independently. Each subject lay supine on the floor near a vertical surface with one leg positioned on the wall and another at rest on the floor. Subjects moved closer to the vertical surface until a comfortable stretch could be felt in the hamstrings. If a position no longer resulted in a stretching sensation experienced by the subject, then the subject moved closer to the vertical surface.

A stabilizing apparatus was used to conduct Active Knee Extension (AKE) tests $[66,67]$. The AKE is a method to indirectly measure hamstring flexibility with high intratester reliability [66]. The stabilizing apparatus ensured pelvic and lower extremity stability during measurement and allowed the measurement to be conducted by a single assessor without assistance. A goniometer with one degree increments was used to measure popliteal angle during the AKE. The AKE test was done before the stretching program, during weekly supervised stretching sessions, and after completion of the stretching program in order to monitor each subjects change in hamstring flexibility over time.

\section{AnyBody Software}

Two methods were used to measure L4/L5 lumbar loading, the Electromyographic (EMG) model developed for this thesis and an inverse dynamics model using the AnyBody software. AnyBody (AnyBody Technology, Aalborg, Denmark) is an inverse dynamics software that makes use of kinematic data from markers and ground reaction from force plates in order to estimate muscle and joint reaction forces.

In order to resolve the issue of infinite solutions existing for balancing muscle forces across a joint (Chapter 2 - Biomechanical Modeling), AnyBody attempts to minimize muscle forces raised to a power. To explain why AnyBody does this, we first discuss what AnyBody describes as linear muscle recruitment:

$$
G=\sum \frac{F i}{N i}
$$

In the above equation, $\mathrm{Fi}$ is the force of a muscle and $\mathrm{Ni}$ is a normalization factor such as physiological cross sectional area. $\mathrm{G}$ in this case is simply the sum of normalized muscle forces. By selecting muscle forces that both balance moments around the joint and minimize $\mathrm{G}$, I get the minimum muscle force necessary to balance moments across a joint which is the most mechanically efficient muscle activation. However, this results in a case where the only muscles active are the ones with the largest moment arms and all other muscles contribute no force to balancing moments around the joint. This issue is 
illustrated in Figure 3-5. This behavior is not found in nature; as multiple muscles work generally in tandem.

Choosing to raise muscle forces to a power is described by AnyBody as polynomial recruitment, shown in Figure 3-6:

$$
G=\sum\left(\frac{F i}{N i}\right)^{n}
$$

Due to the fact that exponential growths exasperate large values of Fi, raising muscle forces to a power generally results in the $\mathrm{G}$ value being minimized when more muscles are included in balancing moments around a joint. AnyBody uses a default value of 3 for $n$, though it can make use of $n$ values up to 5 . AnyBody limits the value of $n$ to 5 for two reasons. First, extremely large values of $n$ result in all muscles being recruited equally, which is also not natural behavior. Secondly, higher orders of $\mathrm{n}$ result in muscle responses within the AnyBody software that are far faster than normal muscle responses. In short, use of a low value polynomial to minimize $\mathrm{G}$ results in mechanically efficient muscle activation while also involving multiple muscles.

In addition to polynomial recruitment, AnyBody makes use of an additional function named MinMax in order to balance muscles around a joint. The MinMax function attempts to minimize the maximum load placed on any muscle, resulting in a high amount of muscle co-activation. By itself, the MinMax function activates and deactivates muscles faster than physiologically possible. A composite function making use of both Polynomial Recruitment and the MinMax function results in well-defined muscle cooperation with appropriate muscle response times [53].

A rigid body dynamic AnyBody model (MoCap_LowerBody, v 6.0.5.4279) was modified to work with the marker set used within the lab. This model included the legs, pelvis, torso, skull, and multiple muscles required to balance moments around joints as shown in Figure 3-7. The arms were not included. A model optimization was done to fit the skeletal model dimensions to the marker set in the lab before an inverse dynamic analysis was run on the model.

\section{Model Calculations}

\section{Building a Force to Surface Electromyography Relationship}

All data analysis of sEMG, marker, and Biodex data was completed in both Visual3D (C-Motion, Germantown, MD) and Matlab (MathWorks, Natick, MA). Visual3D was used to measure torso depth, width, and leg length from each standing trial. Matlab was used to build a muscle force to sEMG relationship for the Erector Spinae (ES), Rectus Abdominus (RA), and External Obliques (EO) of each subject. 


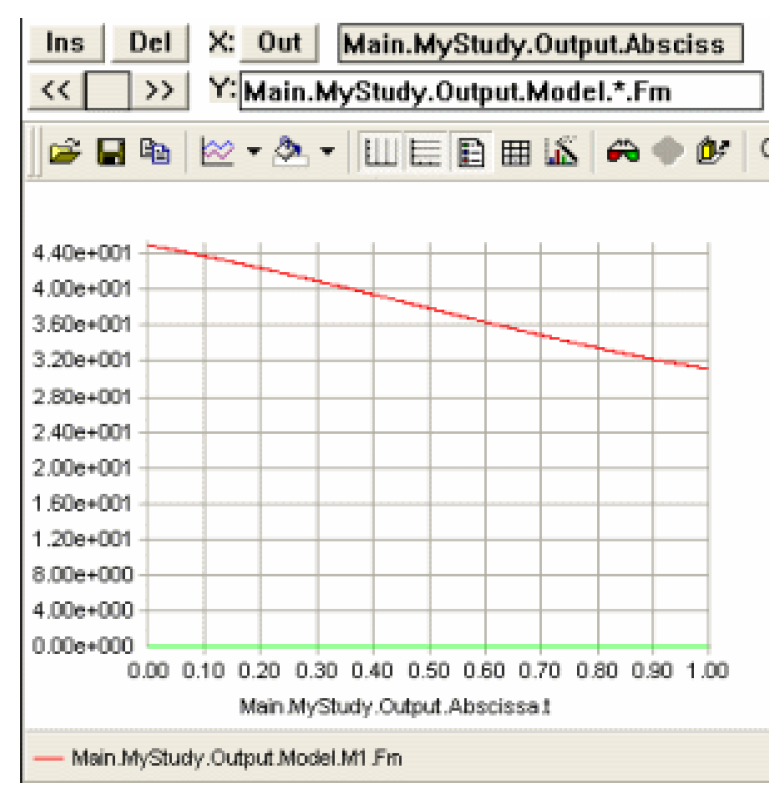

Figure 3-5. Linear Muscle Recruitment.

In linear muscle recruitment, only the muscle closest to the joint (red) shows any activation and those farther from the joint (green) show no activation.

Reprinted with permission. The AnyBody Modeling System Version 6.0.1 AnyBody

Tutorials. AnyBody Tutorials 2013 Sep 2013 [cited 2016 May 24]; Available from:

http://www.anybodytech.com/fileadmin/AnyBody/Docs/Tutorials/ template/FrontPage/F rontPage.html. [53] 


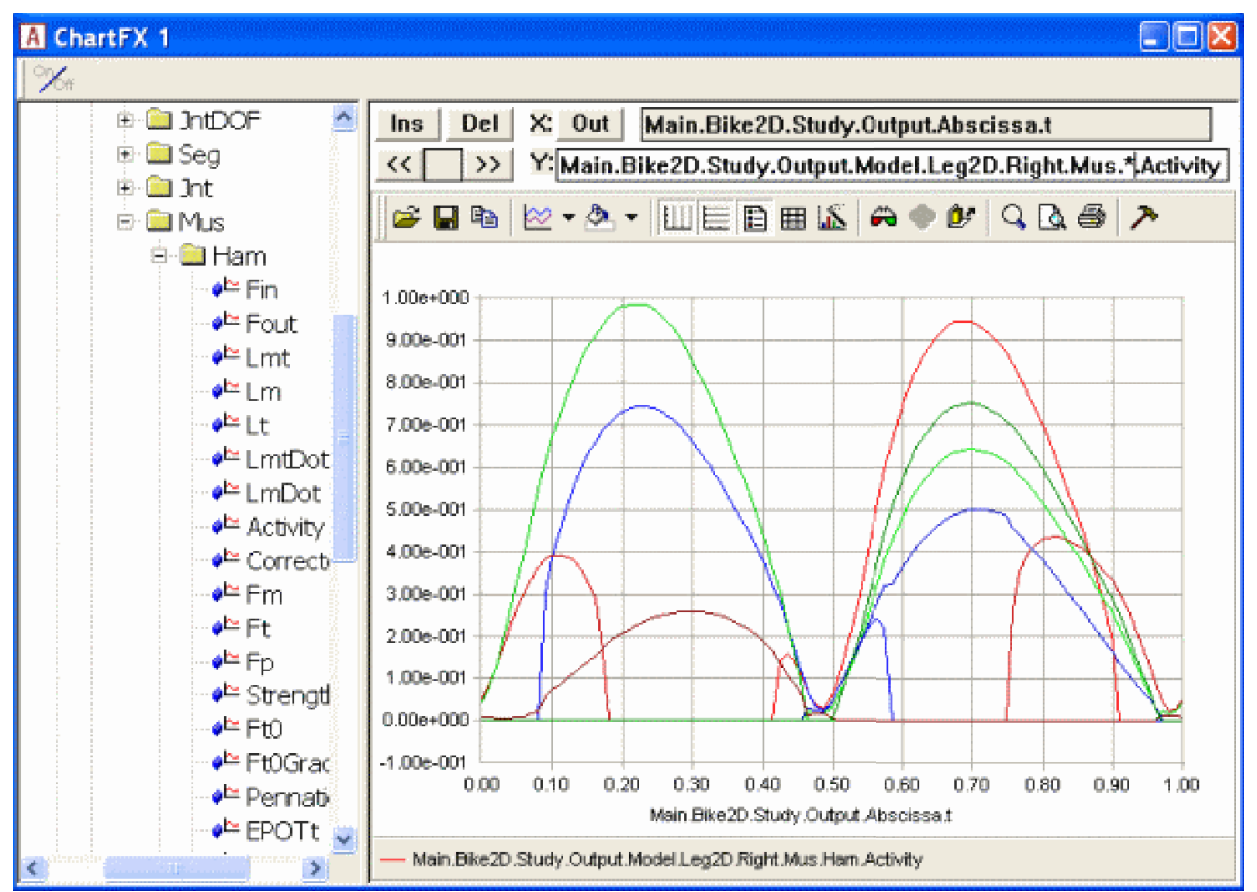

Figure 3-6. Polynomial Muscle Recruitment.

Reprinted with permission. The AnyBody Modeling System Version 6.0.1 AnyBody Tutorials. AnyBody Tutorials 2013 Sep 2013 [cited 2016 May 24]; Available from: http://www.anybodytech.com/fileadmin/AnyBody/Docs/Tutorials/ template/FrontPage/F rontPage.html. [53] 


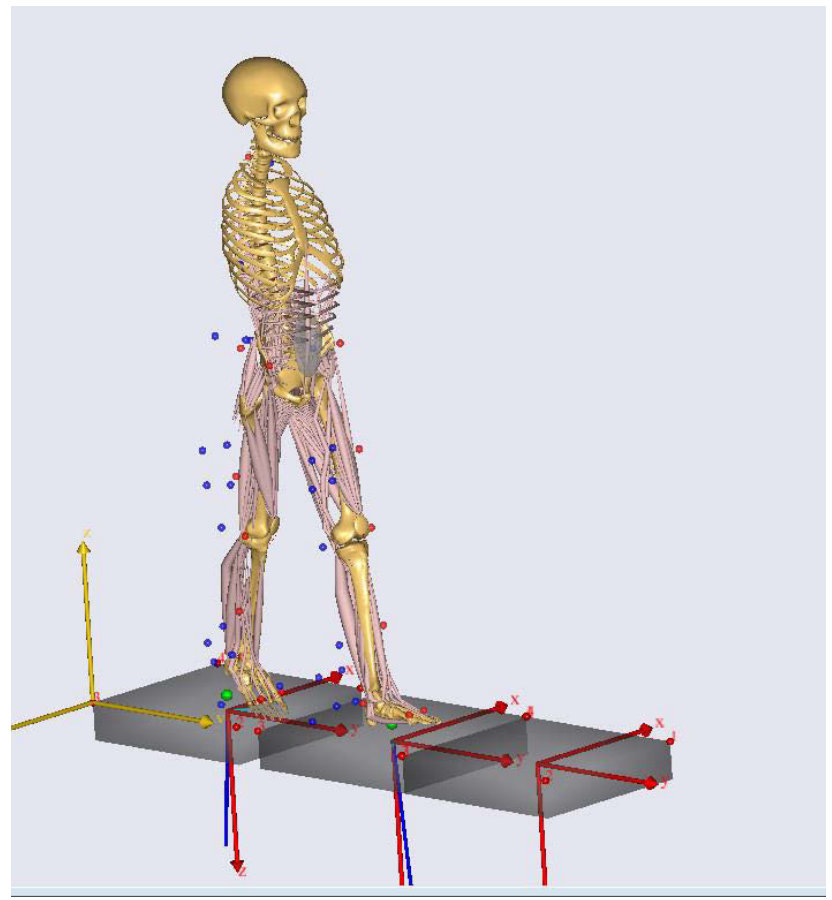

Figure 3-7. AnyBody Lower Body Main Model. 
The average sEMG value during rest trials was subtracted from sEMG signals of voluntary contraction trials for each muscle in order to remove systematic error present sEMG signals. SEMG signals were filtered with a Root Mean Square (RMS) filter with a window width of 51. Use of an RMS filter helped to remove noise spikes in SEMG signals due to movement. Additionally, use of an RMS filter converted all sEMG values into positive values, allowing for a force to sEMG relationship to be built.

Visual3D was used to calculate the subject's trunk depth, width, leg length, and spine length. Subject's trunk width was estimated by the distance between left and right Iliac Crest markers in the transverse plane. Trunk depth was estimated by the distance between Sternum and Sacrum markers in the transverse plane. The spine length was estimated by the distance from a C7 marker to a Sacrum marker in the Z direction. Finally, the leg length was estimated by the distance between an Illiac Crest and Calcaneus marker in the $\mathrm{Z}$ direction.

To more accurately determine the effect of torso mass on the dynamometer torque, a calibration curve of torque versus lever arm angle, shown in Figure 3-8, was calculated for each subject from values recorded before Voluntary Contraction Trials (VCT). Recorded Torque values were also converted into a torso mass value and added to measured scale values, a constant sum of these values confirmed that all of the subject's mass was properly accounted for. All further calculations to produce a muscle force to sEMG relationship was completed in Matlab.

Anthropometric values calculated from Visual3D, subject weight and height, the length of the lever arm used during VCTs, and the body weight effect on dynamometer torque curve were entered into the Matlab code in Appendix B. For each VCT; a QTM, an EMGWorks, and a Biodex file were all entered in Matlab. Within Matlab the X and Yaxis of the laboratory frame was defined differently from the general laboratory frame. The origin was defined at the center of the L4/L5 intervertebral joint. The X-axis ran medio-laterally from the L4/L5 joint and the Y-axis was perpendicular to the X-axis within the transverse plane.

Muscle cross-sectional area and moment arms for each muscle were calculated as a function of subject anthropometry [38]. The moment arms of the Erector Spinae (ES), Rectus Abdominus (RA), and External Obliques (EO) were estimated as a function of torso depth and width [38]. Bilateral muscles were assumed to be equidistant in the $\mathrm{X}$ direction. Cross sectional areas for each muscle were estimated as a function of torso depth and width [38]. Moment arms and cross sectional areas were assumed to be in the transverse plane at the same height as the L4/L5 intervertebral joint. To calculate the muscle direction for the ES and RA muscles, a muscle angle was calculated from the muscle moment arm and the spine length value. The EO muscles were assumed to cross its muscle moment arm at $45^{\circ}$ [48].

Each VCT trial consisted of three cycles of flexion and extension, which were individually split. A lever arm angle of zero from vertical was used to mark the start and ends of a cycle. The time point where the recorded lever arm angle indicated the end of 


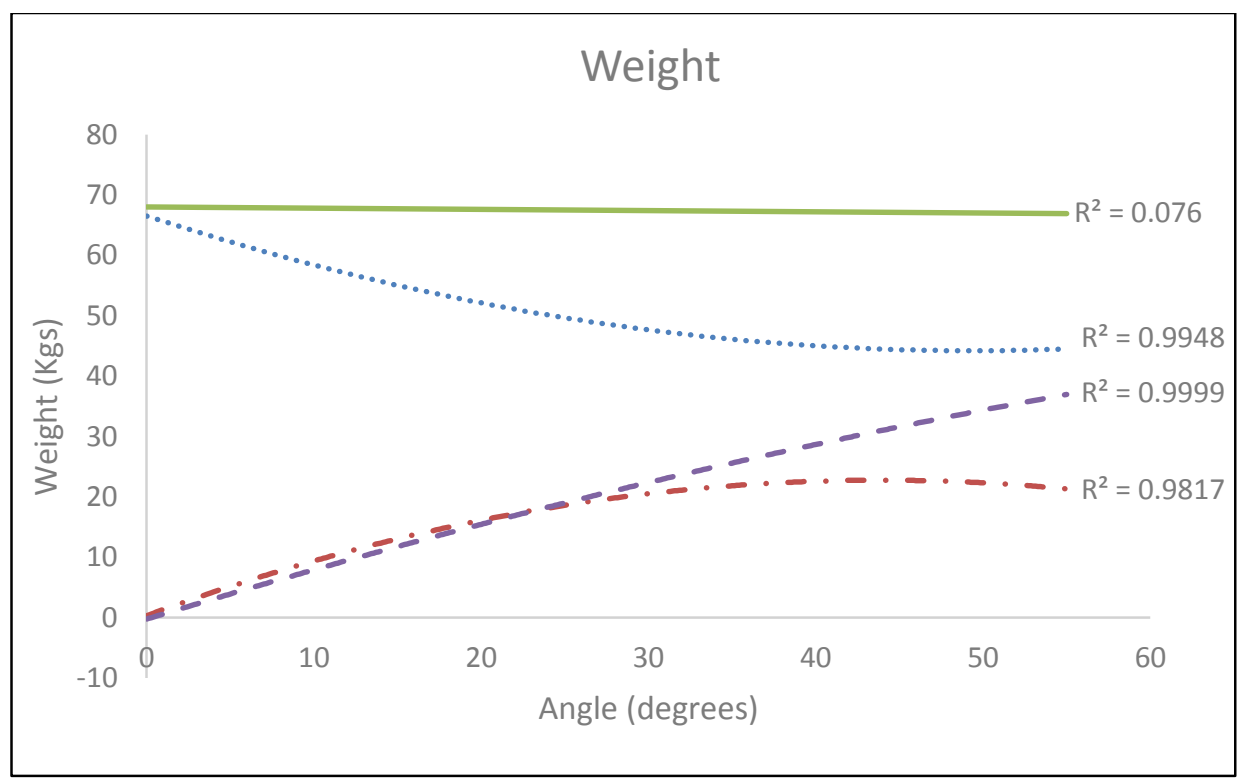

Figure 3-8. Contribution of Torso Weight on Dynamometer Torque.

The body weight supported by the dynamometer (dotted dashed) and the weight accounted for by the scale (dotted) add to a constant total body weight (line). A mathematical assumption of Torso Weight times Sine of the body angle (dashed) does not match up with the body weight supported by the dynamometer, indicating the importance of actually measuring the torso weight on the scale for each angle. 
flexion and the start of extension. Repetitions were then split to divide into flexion and extension. Torque values were corrected for torso weight at all angles throughout extension by adding torque values determined from the torque-angle relationship. To correct for torso weight during flexion these values were subtracted.

A mathematical model, adapted and modified from that described by Schultz et al, was used to create a force to sEMG relationship. Since all VCTs only involved movement in the sagittal plane, bilateral muscle forces were considered equal. For both flexion and extension, the external moments $\left(\mathrm{M}_{\mathrm{E}}\right)$ around the L4/L5 joint:

$$
\sum M_{E}=M_{B}+M_{T W}
$$

Were balanced against the internal moments $\left(\mathrm{M}_{\mathrm{I}}\right)$ resulting in the following equations:

$$
\begin{gathered}
\sum M_{I}=\sum M_{M} \\
M_{B}+M_{T W}=\sum M_{M}
\end{gathered}
$$

Where $\mathrm{M}_{\mathrm{M}}, \mathrm{M}_{\mathrm{TW}}$, and $\mathrm{M}_{\mathrm{B}}$, are the moments around the L4/L5 joint due to trunk muscles, the weight of the torso, and the moment recorded by the Biodex dynamometer respectively. Since the moment on the lever arm due to torso weight was accounted for previously, the previous equation simplifies to:

$$
M_{B}^{*}=\sum M_{M}
$$

Since the ES was the only trunk extensor included in this study, the external moment measured by the isokinetic dynamometer was attributed solely to the ES (MES) and torso weight. The internal moments during flexion included both the EO (MEO) and RA $\left(M_{R A}\right)$. Therefore, the moment balance for extension and flexion respectively are:

$$
\begin{gathered}
M_{B}^{*}=M_{E S} \\
M_{B}^{*}=M_{R A}+M_{E O}
\end{gathered}
$$

To calculate the ES muscle force during an extension cycle, we replace the ES muscle moment above with its force $\left(\mathrm{F}_{\mathrm{ES}}\right)$, angle $\left(\theta_{\mathrm{ES}}\right)$ and moment arm $\left(\mathrm{d}_{\mathrm{ES}}\right)$. Since we know the lever arm of the dynamometer, we can divided the moment by the lever arm $\left(d_{L}\right)$ to get a force.

$$
\frac{M_{B}^{*}}{d_{L}}=F_{E S} * d_{E S} * \cos \theta_{E S}
$$


Solving this equation gives us the muscle force $\mathrm{F}_{\mathrm{ES}}$. To find the maximum ES force for an extension cycle we enter the largest Biodex moment recorded during extension into the previous equation. Since bilateral muscle forces were assumed equal, we divide $\mathrm{F}_{\mathrm{ES}}$ by two to get the force of the RES and LES. Using the maximum sEMG signal recorded from the RES and LES during this same period of time we can create a single $\mathrm{F}_{\mathrm{ES}}$ to $\mathrm{sEMG}$ point for both the RES and LES. This series of calculations is repeated for all extension periods of all VCT's, resulting in nine points for both the RES and LES. An additional point is created from rest sEMG signals of the RES and LES with an assumed muscle force of zero. These points are plotted together to form a muscle force to sEMG relationship for the ES as shown in Figure 3-9.

Calculating the muscle forces of the EO and RA was similar to calculating the ES muscle force, though it involved an extra step. Since two muscles were included for flexion or equation for muscle force is modified for flexion.

$$
\frac{M_{B}^{*}}{d_{L}}=\left(F_{R A} * d_{R A} * \cos \theta_{R A}\right)+\left(F_{E O} * d_{E O} * \cos 45^{\circ}\right)
$$

This equation involves two unknowns. To solve this, we create ratio for $\mathrm{F}_{\mathrm{EO}}$ and $F_{R A}$ from their cross sectional areas $(A)$.

$$
\frac{F_{E O}}{A_{E O}}=\frac{F_{R A}}{A_{R A}}
$$

Inserting this ratio into our equation for flexion allows us to solve for both $F_{R A}$ and $\mathrm{F}_{\mathrm{EO}}$. Once solved, we divide both values by two and build a muscle force to sEMG plot for all four muscles using the same method used for building the RES and LES muscle force to sEMG relationships.

General population trends similar to those produced by Brown et al were also calculated. Muscle forces and corresponding sEMG values were normalized by their maximum muscle force and maximum sEMG value recorded during all three VCT's. These normalized values were then plotted together to form a general population relationship for each muscle as shown in Figure 3-10.

General versus Specific muscle force to sEMG relationships, different contraction velocities, and linear versus logarithmic relationships were compared before muscle force to sEMG relationships were used to calculate L4/L5 compressive loading. The methods and results of these comparisons are detailed in the statistics and results sections of this thesis. 


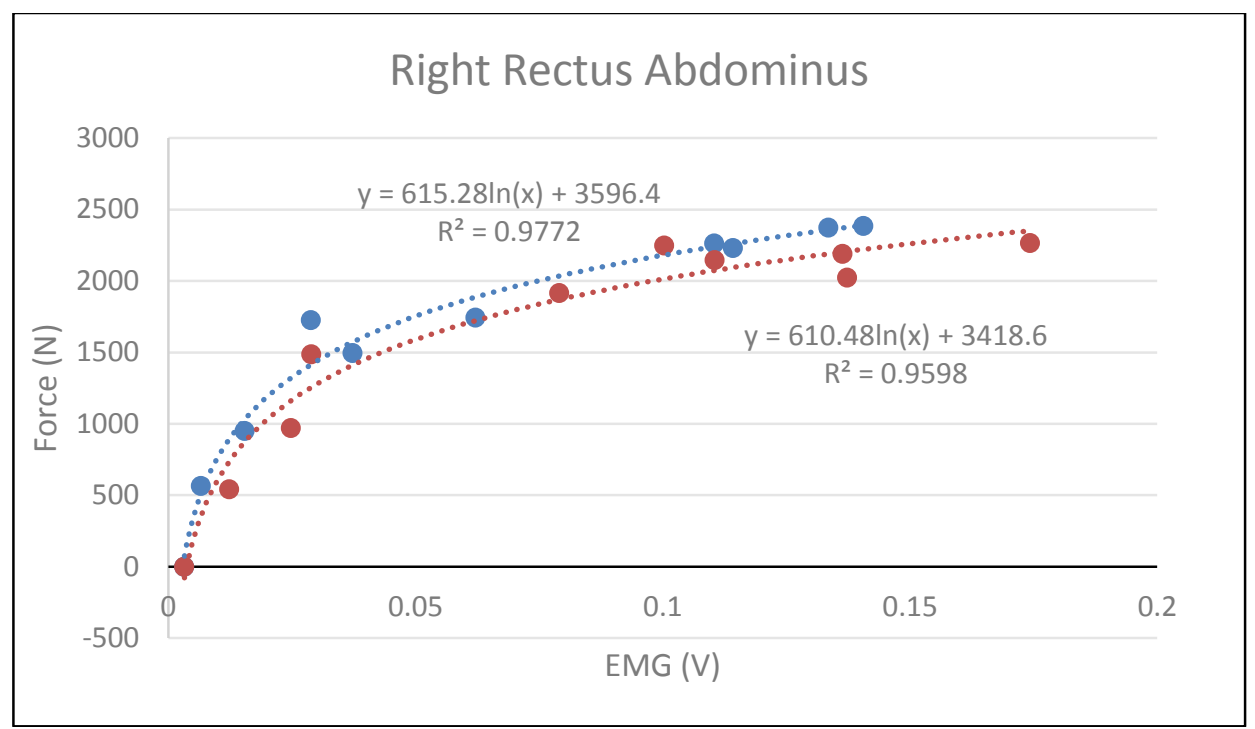

Figure 3-9. Muscle Force to sEMG Relationship for the Right Rectus Abdominus. Muscle force output by the Rectus Abdominus during VCT trials at both $30 \% \mathrm{sec}$ (Blue) and $60 \%$ sec (Red)

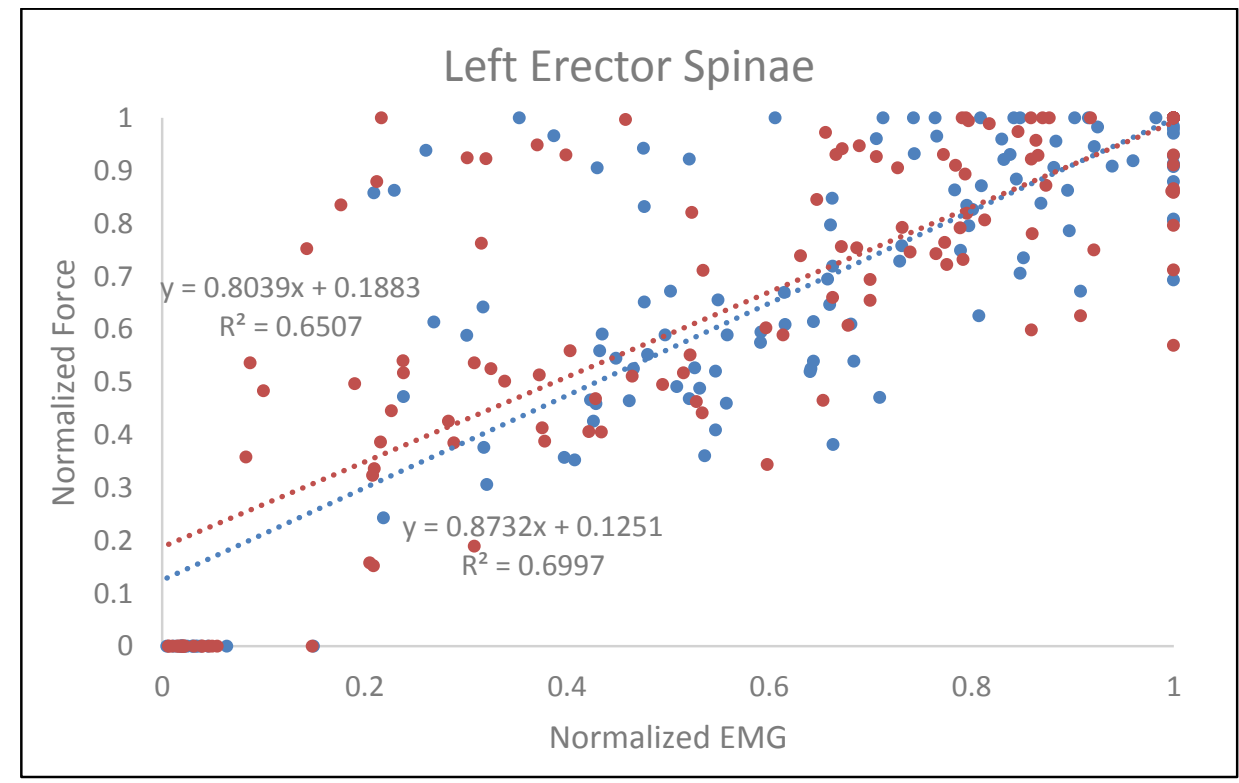

Figure 3-10. General Force to sEMG Relationship for the Left Erector Spinae. Normalized muscle force output by the Left Erector Spinae for all subjects during VCT trials at both $30 \%$ sec (Blue) and $60 \%$ sec (Red) 


\section{Estimating Compressive Loading from Surface EMG Activity}

A Visual3D code shown in Appendix $\mathbf{C}$ was used to build a sEMG model to calculate L4/L5 compressive loading. Inputs to Visual3D included the subject's body mass and height. Subject specific muscle force to sEMG relationships for the RES, LES, RRA, LRA, REO, and LEO muscles were all entered into Visual3D. Finally, a c3d file from QTM of a trial of interest and a resting trial was imported into Visual3D. Straight leg stoop lifting trials were decided as the activity of interest and all further calculations are done for this trial.

Upper body weight was defined as $67 \%$ of the total weight of the subject [68]. Similar to before, sEMG data offset was corrected using the average of rest sEMG values before application of a moving RMS filter. Resting sEMG data was also applied to an RMS filter and the average value post application of the RMS filter was stored as a threshold value.

Muscle forces throughout the trial of interest were calculated by inputting sEMG data during the trial into subject specific muscle force to sEMG relationships previously built. For the ES muscles these relationships were linear, while the relationships for the EO and RA muscles were logarithmic. Relationships built from data at $60 \% \mathrm{sec}$ was used for straight leg stop lifting trials due to the similarity of speed in subjects lifting the $8 \mathrm{~kg}$ medicine ball.

Rest EMG threshold values were also entered into the sEMG relationship to produce a muscle threshold value. Muscle forces below the muscle threshold value were set to zero, as any sEMG activity below rest EMG activity was assumed to correspond to no muscle force. Additionally, logarithmic relationships often produced negative values when sEMG data fell below the rest sEMG signal. Negative muscle force values are impossible and are therefore removed by thresholding.

Once muscle force values were calculated for the trial, the compressive loading on the L4/L5 joint $\left(\mathrm{F}_{\mathrm{J}}\right)$ was calculated by balancing the vertical forces acting upon the joint.

$$
\sum F_{Z}=\left(F_{U B W} * \cos \theta_{U B W}\right)-F_{Z J}+\sum\left(F_{M} * \sin \theta_{M}\right)
$$

Where FUBW is the weight of the upper body and $\theta_{\mathrm{UBW}}$ is the angle the torso made with the vertical measured from marker data. L4/L5 compressive loading data was exported for analysis in excel.

\section{Orthoload Measurements of Compressive Lumbar Loading}

Three subjects from Orthoload's public database were used as a source of in vivo compressive loading data. The data used involved subjects performing a straight leg lifting task as the subjects within this study, though the weight involved was $7 \mathrm{~kg}$. While a 
trial of one individual lifting a $10 \mathrm{~kg}$ weight was available, the trial did not include a full cycle of lifting and replacing the weight, which was necessary for comparison. This data was placed into excel for comparison with AnyBody and the EMG models loading values.

\section{Data Analysis and Statistical Methods}

\section{Force to sEMG Relationships}

Four factors were compared for force to sEMG relationships. These were the relationship linearity (linear vs logarithmic), the specificity (general vs specific), the contraction speed $(30 \% \mathrm{sec}$ vs $60 \% \mathrm{sec})$, and when the relationship was made (Pre or Post hamstring stretching). Since the purpose of this thesis was to reduce error in muscle estimations during EMG modeling, percent error was selected as the variable of interest in comparing relationship factors.

Muscle force and sEMG data was normalized by the maximum muscle force and sEMG values recorded for the same muscle. A general relationship was produced by simultaneously plotting all normalized values and fitting both a linear and logarithmic relationship to the data. Percent error values for each point of each general relationship were then produced by comparing each point with the predicted normalized force value from the trend line. The average percent error for each subject was found by averaging the percent error values of points corresponding to each subject. For specific relationships both linear and logarithmic trend lines were produced and percent errors were calculated between measured and predicted values. Percent error values were also averaged for each subject for specific trials.

All statistics were done in excel unless otherwise specified. A Shapiro-Wilk test was done to test and confirm normality of percent error values within each group. A four way repeated measures Analysis of Variance (ANOVA) between the linearity, specificity, contraction speed, and Pre/Post status was done for each muscle in SPSS (International Business Machines, Armonk, New York).

\section{Comparison between Anybody, and EMG Model}

Loading values from AnyBody, the EMG model, and Orthoload straight leg stoop lifting trials were compared in order to validate the EMG model. For all subjects, lifting cycles were defined as starting when the subject first lifted the weight and ending when they replaced the weight on the ground. An impulse value was calculated for each lifting cycle by integrating the compressive loading value throughout the lifting cycle. Impulse values were normalized by each subject's body weight and averaged for each subject, producing an average impulse value during a lifting cycle for each subject. Two tailed t- 
tests were done between AnyBody and the EMG model in order to detect differences between reported loading values.

\section{Pre and Post Lumbar Loading of Straight Leg Stoop Lifting}

A paired t-test was done on L4/L5 compressive loading data pre and post hamstring stretching for both AnyBody and the EMG model. Of the ten subjects, one subject had not returned for retesting after his hamstring stretching protocol. One subject had a critical marker missing for the AnyBody analysis, resulting in a total of eight subjects included in AnyBody analysis of pre and post compressive loading. Errors in data collection resulted in the inability of the EMG model to analyze both the pre and post data of two subjects, resulting in seven subjects being available for pre and post analysis by the EMG model.

\section{Instrasubject Same Day Variability}

In order to compare the variability in impulse loading due to errors within data calculation, one additional subject was recruited for same day variability testing. The subject underwent an identical protocol as the subjects included in the main study, though the subject did not undergo a stretching protocol and retesting was done only three hours after the initial testing. Three cycles of stoop lifting were compared between the two testing sessions. 


\section{CHAPTER 4. RESULTS}

\section{Force to sEMG Relationships}

A summary of error results for muscle force to sEMG relationships can be found in Table 4-1 and Table 4-2. For all muscles the level of specificity (General vs Specific) had a significant effect on percent error values. In all cases use of a specific trend line resulted in a lower percent error than use of a general trend line. The average decrease in percent error from use of a specific trend line was about $5 \%$.

Both ES muscles showed lower percent errors when a linear relationship was used while all flexor muscles showed a lower percent error when a logarithmic relationship was used. However, of these muscles, only the LRA showed a significant difference ( $\mathrm{p}=$ .023 ) in percent error due to linearity. Two muscles, the RRA and the LEO, had a significant interaction effect between linearity and speed. Both the RRA $(p=0.041)$ and the REO ( $p=0.50)$ showed a significant decrease in percent error for logarithmic relationships are a contraction speed at $30 \%$ sec. The REO also displayed an interaction effect between all factors $(\mathrm{p}=0.025)$, where the subject specific logarithmic post-stretch relationship at $30 \% \mathrm{sec}$ was significantly lower than all other groups. The percent error of this group was $9.2 \%$.

No differences were found in the percent error as an effect of contraction speed or time of measurement. The full results of statistical tests can be found in Appendix D, Tables D-1 through D-6.

\section{Comparison between EMG Model, AnyBody, and Orthoload}

The average body weight of the OrthoLoad subjects $(653 \mathrm{~N} \pm 70)$ was similar to that of our study $(677 \mathrm{~N} \pm 116)$. An example of loading throughout a lifting cycle is shown in Figure 4-1. A summary of results is shown on Table 4-3.

All three models showed similar loading patterns as far as the location of maximums and minimums were concerned. In all models, loading values were at their lowest after the subject finished lifting up the ball and was standing upright while maximum values were typically observed during the process of lifting the ball. Both the EMG model and the AnyBody model showed larger differences between the maximum and minimum values than Orthoload.

The AnyBody model estimated significantly lower impulse values than those estimated by the EMG model $(\mathrm{p}=0.007)$. Compared to the mean impulse value measured by the Orthoload IBR $(713 \mathrm{~N} \pm 108)$, Anybody appeared lower $(463 \mathrm{~N} \pm 139)$. The EMG model showed a larger average impulse estimation $(1007 \mathrm{~N}+484)$ than the Orthoload subjects, due to the large deviation in impulse measurements. 
Table 4-1. Summary of Significance for Relationships.

\begin{tabular}{ll}
\hline Muscle & Factors that had a significant effect on percent error \\
\hline RES & Specificity \\
LES & Specificity \\
RRA & Specificity, Linearity*Speed, Speed*PrePost \\
LRA & Specificity, Linearity \\
REO & Specificity, Linearity*Speed, Specificity*Linearity*Speed*PrePost \\
LEO & Specificity \\
\hline Specificity, linearity, contraction speed, Pre vs Post, and their interaction effects were
\end{tabular}
tested for significance. Which factors had a significant effect on percent error values are listed above. An asterisk (*) indicates that an interaction effect between the two or more variables had an effect on the percent error.

Table 4-2. Summary of Percent Error Values Divided by Measure Factor.

\begin{tabular}{|c|c|c|c|c|c|c|c|c|}
\hline \multirow[b]{2}{*}{ Muscle } & \multicolumn{2}{|c|}{ Specificity } & \multicolumn{2}{|c|}{ Linearity } & \multicolumn{2}{|c|}{$\begin{array}{c}\text { Contraction } \\
\text { speed }\end{array}$} & \multirow[b]{2}{*}{ Pre } & \multirow[b]{2}{*}{ Post } \\
\hline & General & Specific & Linear & Logarithmic & $30 \% / \mathrm{sec}$ & $60 \% / \mathrm{sec}$ & & \\
\hline RES & $22 \%$ & $17 \%$ & $17 \%$ & $22 \%$ & $18 \%$ & $21 \%$ & $18 \%$ & $21 \%$ \\
\hline LES & $23 \%$ & $15 \%$ & $17 \%$ & $22 \%$ & $18 \%$ & $21 \%$ & $17 \%$ & $21 \%$ \\
\hline RRA & $19 \%$ & $16 \%$ & $19 \%$ & $16 \%$ & $16 \%$ & $19 \%$ & $18 \%$ & $17 \%$ \\
\hline LRA & $18 \%$ & $14 \%$ & $18 \%$ & $13 \%$ & $15 \%$ & $17 \%$ & $18 \%$ & $13 \%$ \\
\hline REO & $19 \%$ & $15 \%$ & $18 \%$ & $16 \%$ & $15 \%$ & $19 \%$ & $19 \%$ & $15 \%$ \\
\hline LEO & $18 \%$ & $14 \%$ & $18 \%$ & $15 \%$ & $14 \%$ & $18 \%$ & $16 \%$ & $16 \%$ \\
\hline
\end{tabular}




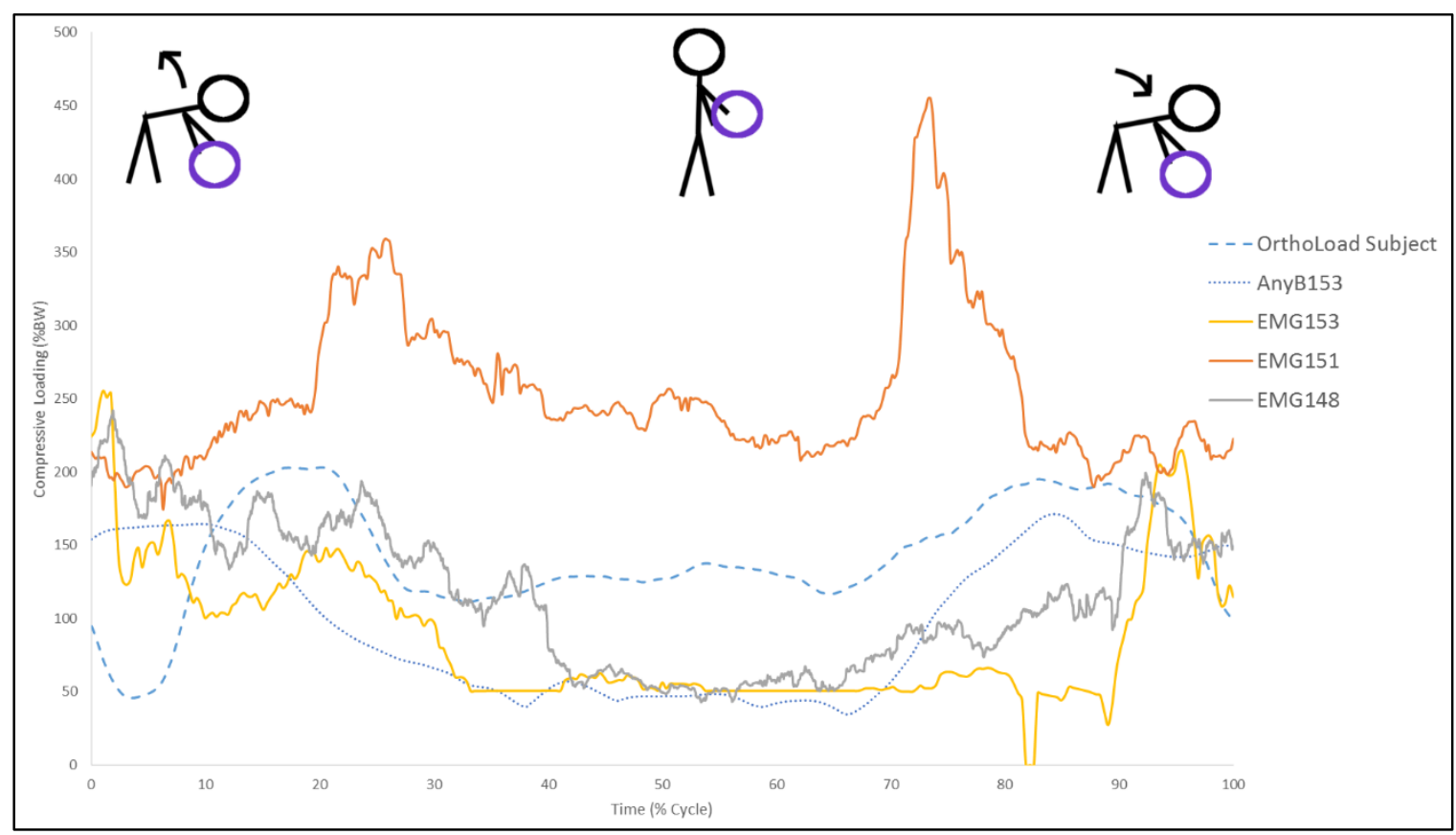

Figure 4-1. $\quad$ Lumbar Loading of All Three Models.

Loading of one Orthoload subject and three subjects included in this study. In two cases the loading pattern of the EMG model (Subjects 148 and 153) appear to be very similar to AnyBody loading. In one case high EMG signals resulted in a subject with abnormally high loading estimates (Subject 151). Impulse values were calculated as the area underneath these curves.

Table 4-3. Summary of Paired t-Tests of Pre and Post Hamstring Stretch Loading.

\begin{tabular}{lcccc}
\hline Model & Pre & Post & Difference & p-Value \\
\hline EMG Model & $1154 \mathrm{~N}$ & $996 \mathrm{~N}$ & $158 \mathrm{~N}$ & 0.42 \\
AnyBody & $484 \mathrm{~N}$ & $421 \mathrm{~N}$ & $63 \mathrm{~N}$ & 0.31 \\
\hline
\end{tabular}


The EMG model showed a substantially larger average impulse estimation $(1007 \mathrm{~N}+484)$, thought this impulse was not found to be different than the Orthoload VBR's $(p=0.104)$, probably due to the large deviation in impulse measurements. This large deviation was reflected in the differences in variance between the EMG model and both AnyBody $(\mathrm{p}<0.001)$ and the Orthoload IBRs $(\mathrm{p}=0.048)$.

\section{Pre and Post Lumbar Loading of Straight Leg Stoop Lifting}

A summary of results can be found on Table 4-3. An F test was done to confirm that there was no difference in the variance of impulse values $(p=0.48)$ in the EMG model between Pre and Post hamstring stretching. Decreases in L4/L5 compressive loading between Pre and Post hamstring stretching trials were measured on average by both the EMG model $(158 \mathrm{~N})$ and the AnyBody model $(63 \mathrm{~N})$. However, for both the EMG model $(p=0.42)$ and the AnyBody model $(p=0.312)$ these differences were not statistically significant.

\section{Instrasubject Same Day Variability}

Intrasubject impulse values normalized by bodyweight seemed to vary between pre $(490 \pm 58)$ and post $(547 \pm 21)$ tests by about $50 \%$ of the subject's body weight throughout the whole trial. The subject also showed a longer time to complete cycles during the post trial $(4.6 \mathrm{~s} \pm .48)$ than during the pre trial $(4.1 \mathrm{~s} \pm .24)$. Despite the higher impulse values observed by post trial, the pre trial showed a higher peak loading $(2.5 \pm$ $.12)$ when compared with the post trial $(2.28+.13)$. 


\section{CHAPTER 5. DISCUSSION}

Our results show that use of patient specific trend lines to define muscle force to sEMG relationships is an important factor in reducing the percent error of L4/L5 compressive loading estimations. Use of specific relationships over general ones reduced percent error in muscle force calculations by an average of $4.5 \%$, this reduction in error can correspond to $\sim 50 \mathrm{~N}$ less error per muscle or a total of $300 \mathrm{~N}$ less error in estimating L4/L5 compressive loading. Despite this, general relationships showed strong correlation coefficients for each muscle, suggesting that a normalized relationship does exist for all subject. In cases where less estimation accuracy is acceptable, MVCs and rest trials alone can be used to define general relationships. This difference was more pronounced when looking at specific groups. Typically, EMG models make use of a single gain value relating muscle force to sEMG, creating a linear relationship between the two that is the same for all subject. For example, use of a subject specific logarithmic relationship as opposed to a linear general relationship resulted in the percent error decreasing from $18 \%$ to $10.6 \%$ in the LRA.

The lack of significant effects due to the linearity of the trend line is surprising given the limitation to the force a muscle can produce, regardless of the amount of motor neuron activity. Because muscle fibers are "all or none" in response to motor neuron signaling, it logically follows that the relationship between electrical activity and muscle force should be logarithmic. In the case of two muscles, the RRA and LEO, there was an interaction effect resulting in a substantially lower percent error for the muscle for to sEMG relationship for logarithmic relationships are $30^{\circ} / \mathrm{sec}$. This would suggest that both the RRA and LEO muscle force to sEMG relationship becomes more linear as contraction speed increases. While no significant effects were found due to linearity for muscles other than the LRA, the linearity selected for each muscle was determined by the linearity that resulted in the least error. For extensor muscles this relationship was linear and for flexors this relationship was logarithmic. Ignoring antagonist muscle activity, these results are consistent with literature.

AnyBody modeling has been shown to estimate physiologically appropriate values for forces in the spine [69]. Our AnyBody consistently reported significantly lower impulse values throughout straight leg stoop lifting pick trials compared to both our EMG model and Orthoload IBR's. There did not seem to be an observable difference in either population body weight and the lower load being lifted by the Orthoload population makes it unlikely that the lower AnyBody loading was a result of differences in the study population.

Our EMG model showed a large variation in impulse values. While a large range of compressive loading values in the spine have been reported in literature, it is possible that this impulse variation was partially due to subject variation. Previous studies have shown that trunk muscle activation can vary significantly for subjects performing the same task [70,71]. Our model made use of only six muscles to estimate compressive loading, resulting in all the extensor moment being attributed to the ES muscles during 
MVC trials. It's possible that subject variation in muscle activation of extensor muscles resulted in extremely higher compressive loads in subjects who show significantly higher ES activation relative to other extensors. Since I am primarily concerned with how subjects L4/L5 compressive loading changed after a six week stretching protocol, our model is still applicable for studying L4/L5 compressive loading changes due to hamstring flexibility. Despite this large variance, our model did report compressive loading values that reflect those reposted in the literature.

Both our EMG model and the AnyBody model failed to show a differences in L4/L5 compressive loading pre and post hamstring stretching. For all but one subject both models showed agreement in the direction of changes in L4/L5 loading pre and post hamstring stretching. Regardless, neither model detected any difference in compressive loading due to hamstring stretching. While I hypothesized that L4/L5 compressive loading would decrease with hamstring stretching, it's possible that hamstring stretching does not alter total L4/L5 compressive loading, but shifts it towards the anterior of the disk. This difference would not be able to be measured with either the EMG or AnyBody model.

This thesis was subject to some limitations. Estimation of muscle cross sectional area and muscle moment arms were estimated via anthropometric data, which may not accurately reflect the subject's actual muscle cross sectional areas and moment arms. Larger moment arms for muscles would result in lower forces needed to balance forces around the L4/L5 joint which would result in lower compressive loads. Cross sectional areas of muscles were used to "distribute" muscle force amongst muscles participating in the same activity. Both of these issues can be resolved by use of an imaging device to properly measure both these quantities.

Inclusion of only six muscles means that extension and flexion force had to be solely attributed to these muscles. Due to differing muscle moment arms, attribution of forces to only a few muscles can result in large changes in the L4/L5 compressive loading. For example, the Latissimus Dorsi aids in extension of the back and has a larger moment arm than the ES. Inclusion of both when creating force to sEMG relationships would result in less force being attributed to both muscles together than to the ES alone when not factoring in the Latissimus Dorsi. This would result in lower L4/L5 compressive loading estimates during straight leg stoop lifting trials. These differences, along with the differences due to cross sectional and moment arm estimations, would change the muscle force to sEMG relationships by division of a constant and therefore would not affect the overall shape or percent error values of the trend lines.

The most significant limitation in constructing my muscle force to sEMG relationships is that I currently do not factor in the effect of antagonist muscle activity into our muscle force calculations. Brown et al reported that non-linear muscle moments to $\mathrm{sEMG}$ relationships became more linear once antagonist muscle activity was accounted for. However, I did not observe antagonist muscle activity as high as that reported by Brown et al, who reported antagonist activity as high as $298 \%$ of agonist muscle activity. Visually, our antagonist muscle activity appeared to be at most $50 \%$ of 
agonist muscle activity. This difference in observed antagonist activity may be due to differences in contraction methods. A previous study has shown that isokinetic contractions have been shown to have reduced antagonist activation compared to isotonic contractions in the knee. It is possible these differences may apply to isometric contractions, though no reported differences exist to the author's knowledge. Additionally, it's possible that use of the dynamometer lever arm functioned to replace some of the necessary antagonist muscle activity.

Finally, the total length of testing is about three hours. This length of time makes of difficult to redo MVC trials if a marker or electrode falls off halfway through testing. In the case of two subjects the force to EMG relationship had to be rescaled due to an electrode falling off. This may not be accurate as electrode placement might influence the force to EMG curve shape. This limitation is one of the easier ones to resolve, as it only requires securing the electrodes.

While my model did have a difference in impulse loading for the same subject pre and post, this loading difference was only $10 \%$ of the total loading. Additionally, when normalized for the time spent completing the loading cycle, these values were the same for pre $(118.8 \% \mathrm{BW} / \mathrm{s})$ and post $(118.0 \% \mathrm{BW} / \mathrm{s})$ loading, which suggests that the difference in loading was mainly due to differences in the amount of time the subject spent lifting and replacing the medicine ball.

In conclusion, my EMG model presents the first major steps towards development of a subject specific model for measuring L4/L5 compressive loading. My model reports compressive loading values similar to those reported in literature with a high intra subject repeatability, though it needs improvement in consistency for different subjects. Inclusion of more muscles and antagonist muscle activity during VCT trials will likely improve the accuracy of the EMG model. Finally, my model shows the importance in developing subject specific relationships to compare muscle force to sEMG activity. 


\section{LIST OF REFERENCES}

1. Ehrlich, G.E., Low Back Pain. Bulletin of the World Health Organization, 2003. 81: p. 671-676.

2. Meucci, R.D., A.G. Fassa, and N.M. Faria, Prevalence of chronic low back pain: systematic review. Rev Saude Publica, 2015. 49(73).

3. Taylor, J.B., et al., Incidence and risk factors for first-time incident low back pain: a systematic review and meta-analysis. Spine J, 2014. 14(10): p. 2299-319.

4. Andersson, G.B., Epidemiological features of chronic low-back pain. Lancet, 1999. 354(9178): p. 581-5.

5. Gou, S., et al., Stem cell therapy for intervertebral disk regeneration. Am J Phys Med Rehabil, 2014. 93(11 Suppl 3): p. S122-31.

6. Kaplan, W., et al. Priority Medicines for Europe and the World 2013 Update. 2013. 2016, 165-168.

7. Vadala, G., et al., Intervertebral disc regeneration: from the degenerative cascade to molecular therapy and tissue engineering. J Tissue Eng Regen Med, 2015. 9(6): p. 679-90.

8. Coenen, P., et al., The cotribution of laod magnitude and number of load cycles to cumulative low-back load estimators: A study based on in-vitro compression data. Clin Biomech (Bristol, Avon), 2012. 27(10): p. 1083-6.

9. Deyo, R.A. and J.N. Weinstein, Low Back Pain. N Engl J Med, 2001. 344(5): p. 363-70.

10. Sano, A., et al., Body mass index is associated with low back pain in childhood and adolescence: a birth cohort study with a 6-year follow-up in Niigata City, Japan. Eur Spine J, 2015. 24(3): p. 474-81.

11. Oliveira, V.C., et al., Risk Factors for low back pain: insights from a novel casecontrol twin study. Spine J, 2015. 15(1): p. 50-7.

12. Gupta, N., et al., Is objectively measured sitting time associated with low back pain? A cross-sectional investigation in the NOMAD study. PLoS One, 2015. 10(3).

13. Nordin, M. and V.H. Frankel, Basic Biomechanics of the Musculoskeletal System. 3 ed, ed. D. Ledger. 2001, Baltimore, Maryland: Lippincott Williams \& Wilkins.

14. Halbertsma, J.P., et al., Extensibility and stiffness of the hamstrings in patients with nonspecific low back pain. Arch Phys Med Rehabil, 2001. 82(2): p. 232-8.

15. Spinal Fusion. [Webpage] 20102010 [cited 2016 May 22]; Available from: http://orthoinfo.aaos.org/topic.cfm?topic $=\mathrm{a} 00348$.

16. Rohlmann, A., F. Graicen, and G. Bergmann, Methods for Avoiding or Reducing High Spinal Loads in Everyday Life. Journal of Novel Physiotherapy and Physical Rehabilitation, 2014: p. 25-9.

17. Baliga, S., K. Treon, and N.J.A. Craig, Low Back Pain: Current Surgical Approaches. Asian Spine J, 2015. 9(4): p. 645-57.

18. Lange, U., et al., Anterior vertebral body replacement with a titanium implant of adjustable height: a prospective study. Eur Spine J, 2006. 16(2): p. 161-172.

19. Chen, Q. and G. Thouas, Biomaterials A Basic Introduction. 2015, Boca Raton, FL: CRC Press. 692. 
20. Kløjgaard, M.E., et al., Patient preferences for treatment of low back pain-a discrete choice experiment. Value health, 2014. 17(4): p. 390-6.

21. Benyamin, R.M., et al., The effectiveness of lumbar interlaminar epidural injections in managing chronic low back and lower extremity pain. Pain Physician, 2015. 15(4): p. E363-404.

22. A, S.R. Lumbar Epidural Steroid Injections for Low Back Pain and Sciatica 2007 Jul 2007 [cited 2016 June]; Available from: http://www.spinehealth.com/treatment/injections/lumbar-epidural-steroid-injections-low-backpain-and-sciatica.

23. Bredow, J., et al., Conservative treatment of nonspecific, chronic low back pain: Evidence of the efficacy - a systematic literature review. Orthopade, 2016.

24. Han, H.I., H.S. Choi, and W.S. Shin, Effects of hamstring stretch with pelvic control on pain and work ability in standing workers. J Back Musculoskelet Rehabil, 2016.

25. Srbinoska, H., et al., Correlation between back shape and spinal loads. J Biomech, 2013`. 46(11): p. 1972-5.

26. Jenkins, D.B., Functional Anatomy of the Limbs and Back. Eighth ed. 2002, Philidelphia, Pennsylvania: W.B. Saunders Company. 441.

27. Edoarado. 2011 [cited 2016 May 30]; Available from: https://commons.wikimedia.org/wiki/File:Anatomical_Planes-en.svg.

28. Gray, H., Anatomy of the Human Body. 20 ed, ed. W.'.H. Lewis. 1918, Philadelphia and New York: Lea and Febiger.

29. Apazidis, A., et al., The prevalence of transitional vertebrae in the lumbar spine. Spine J, 2011. 11(9): p. 858-62.

30. Luboga, S., Supernumerary lumbar vertebrae in human skeletons at the Galloway Osteological Collection of Makerere University, Kampala. East Afr Med J, 2000. 77(1): p. 16-9.

31. Biluts, H., T. Munie, and M. Abebe, Review of lumbar disc diseases at Tikur Anbessa Hospital. Ethiop Med J, 2012. 50(1): p. 57-65.

32. Mostofi, K., Total Disc Arthroplasty for Treating Lumbar Degenerative Disc Disease. Asian Spine J, 2015. 9(1): p. 59-64.

33. Levangie, P.K. and C.C. Norkin, Joint Structure and Function. Fifth Edition ed, ed. M. Duffield. 2011, Philidelphia, PA: F.A. Davis Company. 588.

34. Kirkaldy-Willis, W.H., et al., Pathology and pathogenesis of lumbar spondylosis and stenosis. Spine, 1978. 3(4): p. 319-28.

35. OpenStax. Anatomy \& Physiology. 2014 [cited 2016 May 5]; Available from: http://cnx.org/contents/14fb4ad7-39a1-4eee-ab6e-3ef2482e3e22@,6.27

36. BrusBlaus. Pelvis. Blausen Gallery 20142013 [cited 2016 May 30]; Available from:

https://en.wikiversity.org/wiki/Wikiversity_Journal_of_Medicine/Blausen_gallery 2014.

37. Rectus Abdominis. 2005 [cited 2016 May 30]; Available from: https://commons.wikimedia.org/wiki/File:Rectus_abdominis.png.

38. Granata, K.P. and W.S. Marras, An EMG-assisted model of laods on the lumbar spine during asymmetric trunk extensions. J Biomech, 1993. 26(12): p. 1429-38. 
39. Gagnon, D., C. Larviere, and P. Loisel, Comparative ability of EMG, optimization, and hybrid modeling approaches to predict trunk muscle forces and lumber spine loading during dynamic sagittal plane lifting. Clin Biomech (Bristol, Avon), 2001. 16(5): p. 359-72.

40. An, K.N., R.L. Linscheid, and P.W. Brand, Correlation of physiological crosssectional areas of muscle and tendon. J Hand Surg Br, 1991. 16(1): p. 66-7.

41. Castanharo, R., M. Duarte, and S. McGill, Corrective sitting strategies: An examination of muscle activity and spine loading. J Electromyogr Kinesiol, 2014. 24(1): p. 114-9.

42. Rohlmann, A., et al., How does the way a weight is carried affect spinal loads? Ergonomics, 2014. 57(2): p. 262-70.

43. Brown, S.H.M. and S.M. McGill, Co-activation alters the linear versus nonlinear impression of the EMG-torque relationship of trunk muscles. J Biomech, 2008. 41(3): p. 491-7.

44. Rohlmann, A., et al., Spinal Loads during Post-Operative Phyiotheraputic Exercises. PLoS One, 2014. 9(7).

45. Synthes Spine 510(k) Premarket Notification Synthes SynExTm Spacer, D.o.H.H. Services, Editor. 2001, Food and Drug Administration.

46. Rohlmann, A., et al., An instrumented implant for vertebral body replacement that measures loads in the anterior spinal column. Med Eng Phys, 2006. 29(5): p. 580-5.

47. Synex System. An expandable vertebral body replacement device. Technical Guide. 2001; Available from: http://www.synthes.com/MediaBin/US\%20DATA/Product\%20Support\%20Mater ials/Technique \%20Guides/SPTGSynexJ3704G.pdf.

48. Schultz, A. and G. Andersson, Analysis of Loads on the Lumbar Spine. Spine, 1981. 6(1): p. 76-82.

49. Schultz, A., et al., Loads on the lumbar spine. Validation of a biomechanical analysis by measurements of intradiscal pressures and myoelectric signals. J Bone Joint Surg Am, 1982. 64(5): p. 713-20.

50. Schellenberg, F., et al., Review of Modelling Techniques for In Vivo Muscle Force Estimation in the Lower Extremities during Strenght Training. Comput Math Methods Med, 2015.

51. D'lima, D.D., et al., Knee joint forces: prediction, measurement, and significance. Proc Inst Mech Eng H, 2012. 226(2): p. 95-102.

52. Erdemir, A., et al., Model-based estimation of muscle forces exerted during movements. Clin Biomech, 2007. 22(2): p. 131-54.

53. The AnyBody Modeling System Version 6.0.1 AnyBody Tutorials. AnyBody Tutorials 2013 Sep 2013 [cited 2016 May 24]; Available from: http://www.anybodytech.com/fileadmin/AnyBody/Docs/Tutorials/ template/Fron tPage/FrontPage.html.

54. Ahamed, N.U., et al., Effects of anthropometric variables and electrode placement on the SEMG activity of the biceps brachii muscle during submaximal isometric contraction in arm wrestling. Biomed Tech (Berl), 2013. 58(5): p. 47588. 
55. Gerdle, B., et al., The influences of muscle fibre proportions and areas upon EMG during maximal dynamic knee extension. Eur J Appl Physiol, 2000. 81(1-2): p. 210.

56. Svebak, S., et al., Electromyographic activation and proportion of fast versus slow twitch muscle fibers: A genertic disposition for psychogenic muscle tension. Int J Psychophysiol. 15(1): p. 43-9.

57. Huebner, A., et al., Re-evaluation of the amplitude-force relationship of trunk muscles. J Biomech, 2015. 48(6): p. 1198-205.

58. Anders, C., et al., Evaluation of the EMG-force relationship of trunk muscles during whole body tilt. J Biomech, 2008. 41(2): p. 333-9.

59. McGill, S.M. and R.W. Norman, Partitioning of the L4-L5 dynamic moment into disc, ligamentous, and muscular components during lifting. Spine (Phila $\mathrm{Pa}$ 1976), 1986. 11(7): p. 666-78.

60. Vera-Garcia, F.J., et al., Effects of different levels of torso coactivation on trunk muscular and kinematic responses to posteriorly applied sudden loads. Clin Biomech (Bristol, Avon), 2006. 21(5): p. 443-55.

61. Bigland, B. and O.C.J. Lippold, The relation between force, velocity and integrated electrical activity in human muscles. J Physiol, 1954. 123(1): p. 214224.

62. Mannion, A.F. and P. Dolan, The effects of muscle length and force output on the EMG power spectrum of the erector spinae. J Electromyogr Kinesiol, 1996. 6(3): p. $159-68$.

63. Taylor, N.A., et al., Static and dynamic assessment of the Biodex dynamometer. Eur J Appl Physiol Occup Physiol, 1991. 62(3): p. 180-8.

64. Drouin, J.M., et al., Reliability and validity of the Biodex system 3 pro isokinetic dynamometer velocity, torque and position measurements. Eur J Appl Physiol, 2004. 91(1): p. 22-9.

65. Decoster, L.C., et al., Standing and Supine Hamstring Stretching Are Equally Effective. J Athl Train, 2004. 39(4): p. 330-334.

66. Hamid, M.S.A., M.R.M. Ali, and A. Yusof, Interrater and Intrarater Reliability of the Active Knee Extension (AKE) Test among Healthy Adults. J Phys Ther Sci, 2013. 25(8): p. 957.

67. DePino, G.M., W.G. Webright, and B.L. Arnold, Duration of maintained hamstring flexibility after cessation of an acute static stretching protocol. J Athl Train, 2000. 35(1): p. 56-59.

68. Winter, D.A., BIOMECHANICS AND MOTOR CONTROL OF HUMAN MOVEMENT. 4th Edition ed. 2009, Hoboken, New Jersey: John Wiley \& Sons.

69. Zee, M.d., et al., A generic detailed rigid-body lumbar spine model. J Biomech, 2007. 40(6): p. 1219-27.

70. Frere, J. and F. Hug, Between-subject variability of muscle synergies during a complex motor skill. Front Comput Neurosci, 2012. 6(99).

71. Kristiansen, M., et al., Inter-subject variability of muscle synergies during bench press in power lifters and untrained individuals. Sc and J Med Sci Sports, 2015. 25(1): p. 89-97. 


\section{APPENDIX A. INSTITUTIONAL REVIEW BOARD}

\begin{tabular}{lr}
\hline THE UNIVERSITY OF TENNESSEE \\
Health Science Center
\end{tabular}

March 18, 2015

Bill Mihalko, M.D., Ph.D.

UTHSC - COM - Orthopaedic Surgery \& Biomedical Engineering

E226 Coleman College of Medicine Building

956 Court Avenue

Memphis, TN 38163

Re: 15-03692-FB UM

Study Title: Hamstring Tightness and Pelvic Position during Activity

Dear Dr. Mihalko:

The IRB has received your written acceptance of and/or responses dated 03/18/2015 and $3 / 11 / 2015$ to the provisos outlined in our correspondence of $3 / 12 / 2015$ and $3 / 4 / 2015$ concerning the application for the above referenced project. The IRB has reviewed these materials and determined that they comply with proper consideration for the rights and welfare of human subjects and the regulatory requirements for the protection of human subjects. Therefore, this letter constitutes full approval by the IRB of your application Version 1.3 including:

- Recruitment script, dated 3/2/2015 (stamped IRB approved 3/18/2015)

- Main consent form, dated 3/10/2015 (stamped IRB approved 3/18/2015)

The UTHSC IRB stamped-approved consent form must be used to enroll prospective subjects in the study.

Approval of this study will be valid from $3 / 18 / 2015$ to $3 / 4 / 2016$.

The IRB has determined that the informed consent form, incorporating the authorization of subjects to use their protected health information in research, complies with the federal privacy regulations as specified in 45 CFR 160 and 45 CFR 164. 
In the event that subjects are to be recruited using solicitation materials, such as brochures, posters, web-based advertisements, etc., these materials must receive prior approval of the IRB. Any revisions in the approved application must also be submitted to and approved by the IRB prior to implementation. In addition, you are responsible for reporting any unanticipated serious adverse events or other problems involving risks to subjects or others in the manner required by the local IRB policy.

Finally, re-approval of your project is required by the IRB in accord with the conditions specified above. You may not continue the research study beyond the time or other limits specified unless you obtain prior written approval of the IRB.

Sincerely,

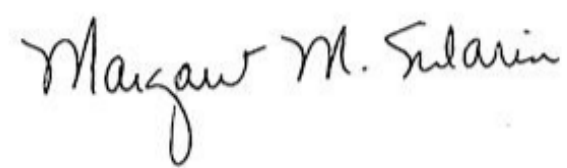

Signature applied by Margaret M Sularin on 03/18/2015 01:44:54 PM CDT

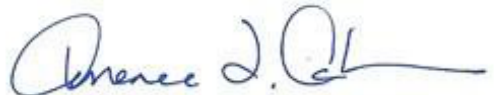

Signature applied by Terrence F Ackerman on 03/18/2015 01:45:37 PM CDT

Margaret M. Sularin, LMSW, RDN, CCRP, CIM, CIP Senior Regulatory Specialist

UTHSC IRB
Terrence F. Ackerman, Ph.D.

Chairman

UTHSC IRB 


\section{APPENDIX B. MATLAB CODE}

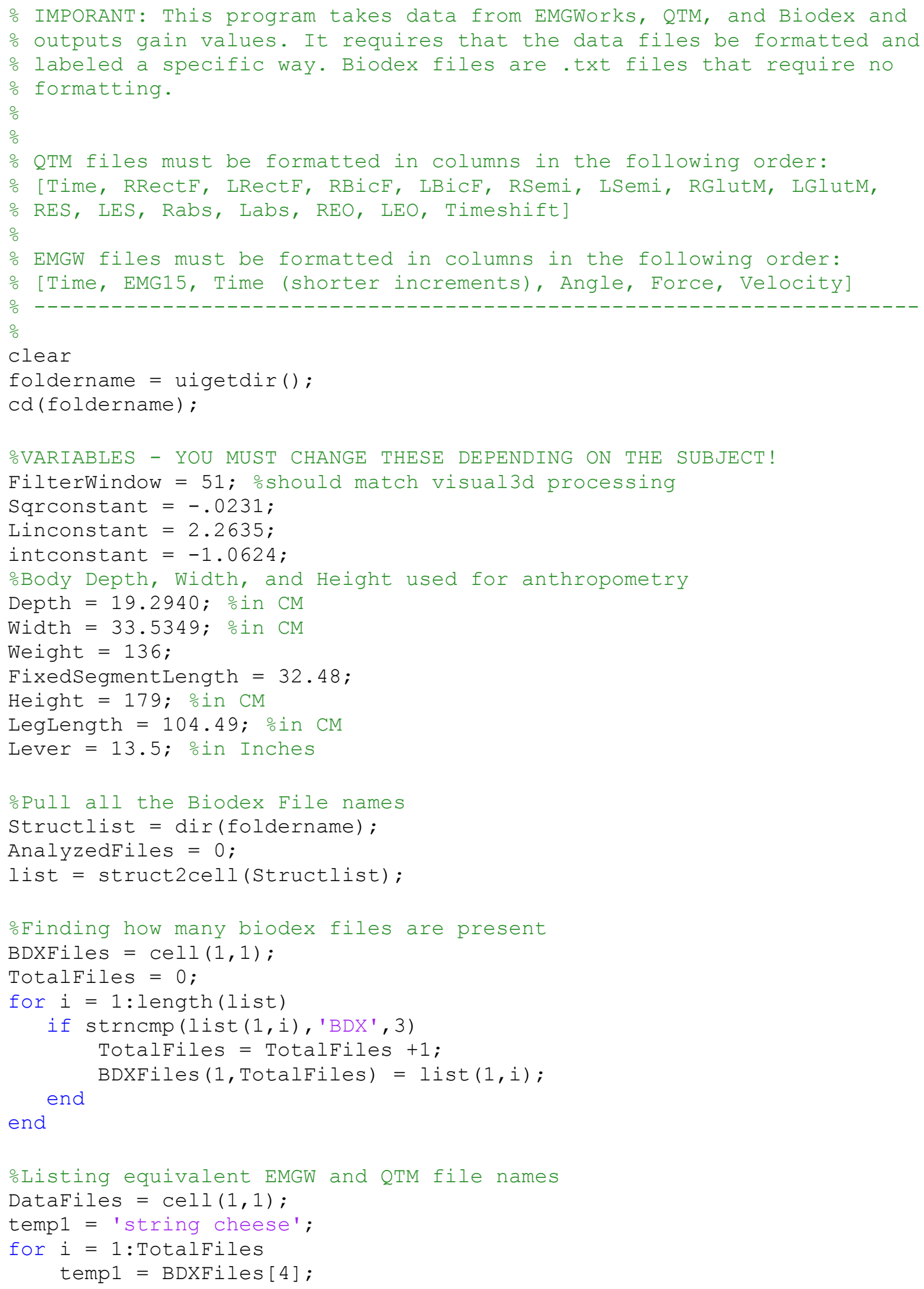




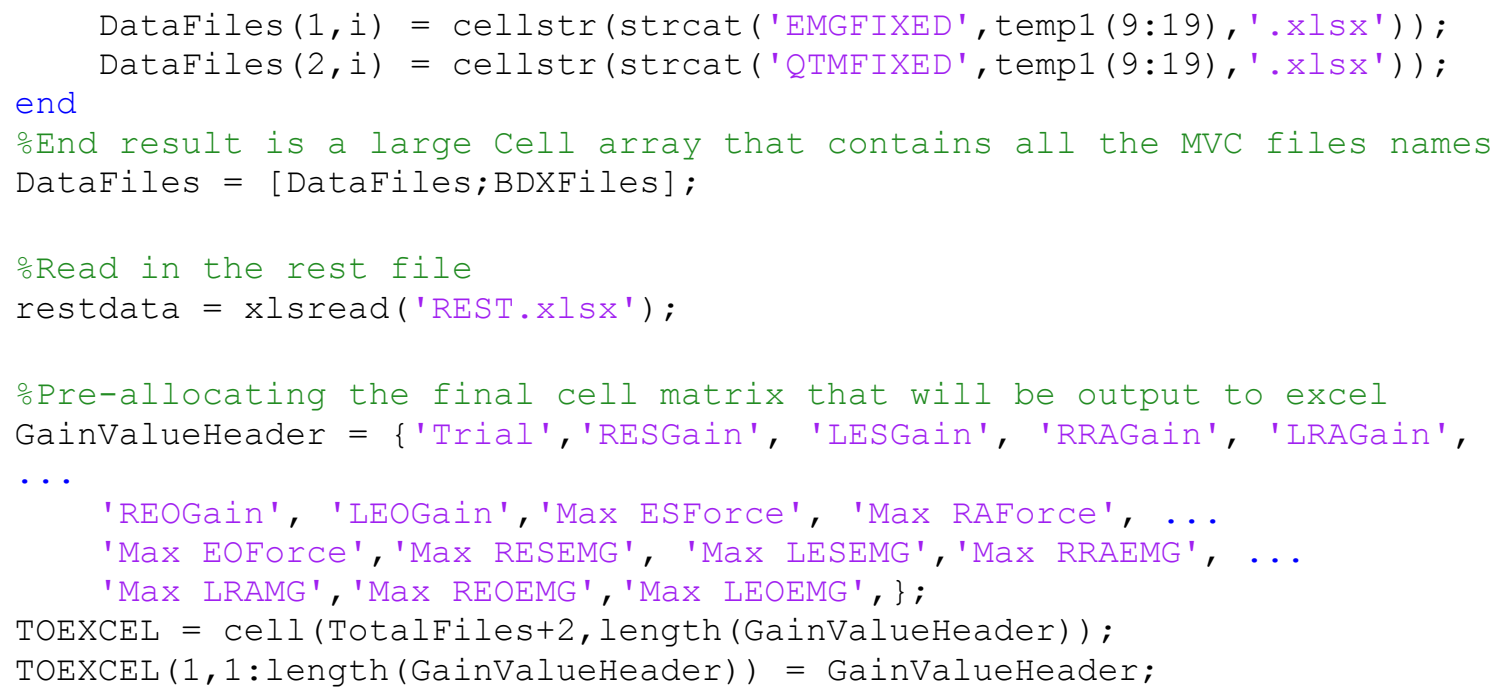




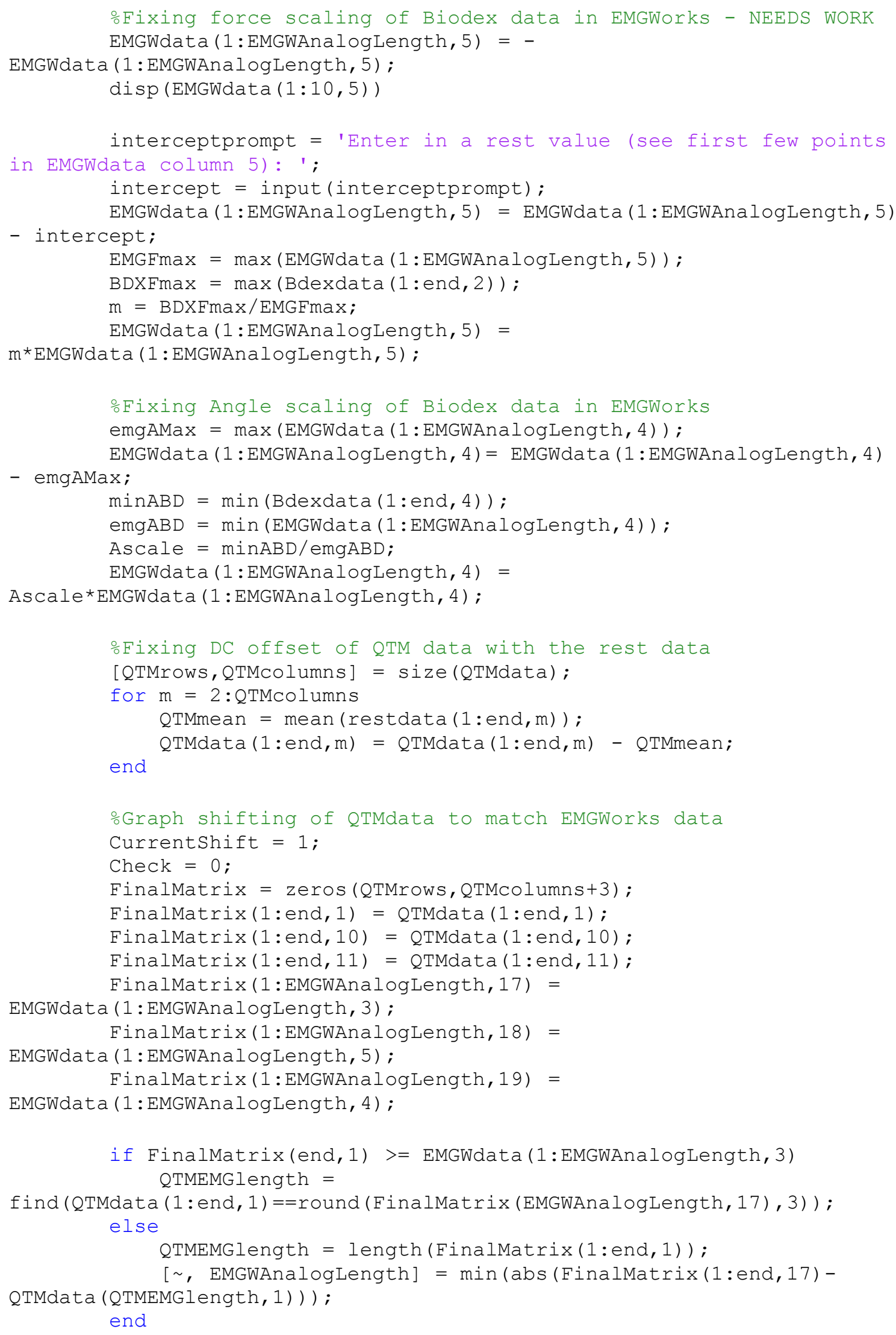




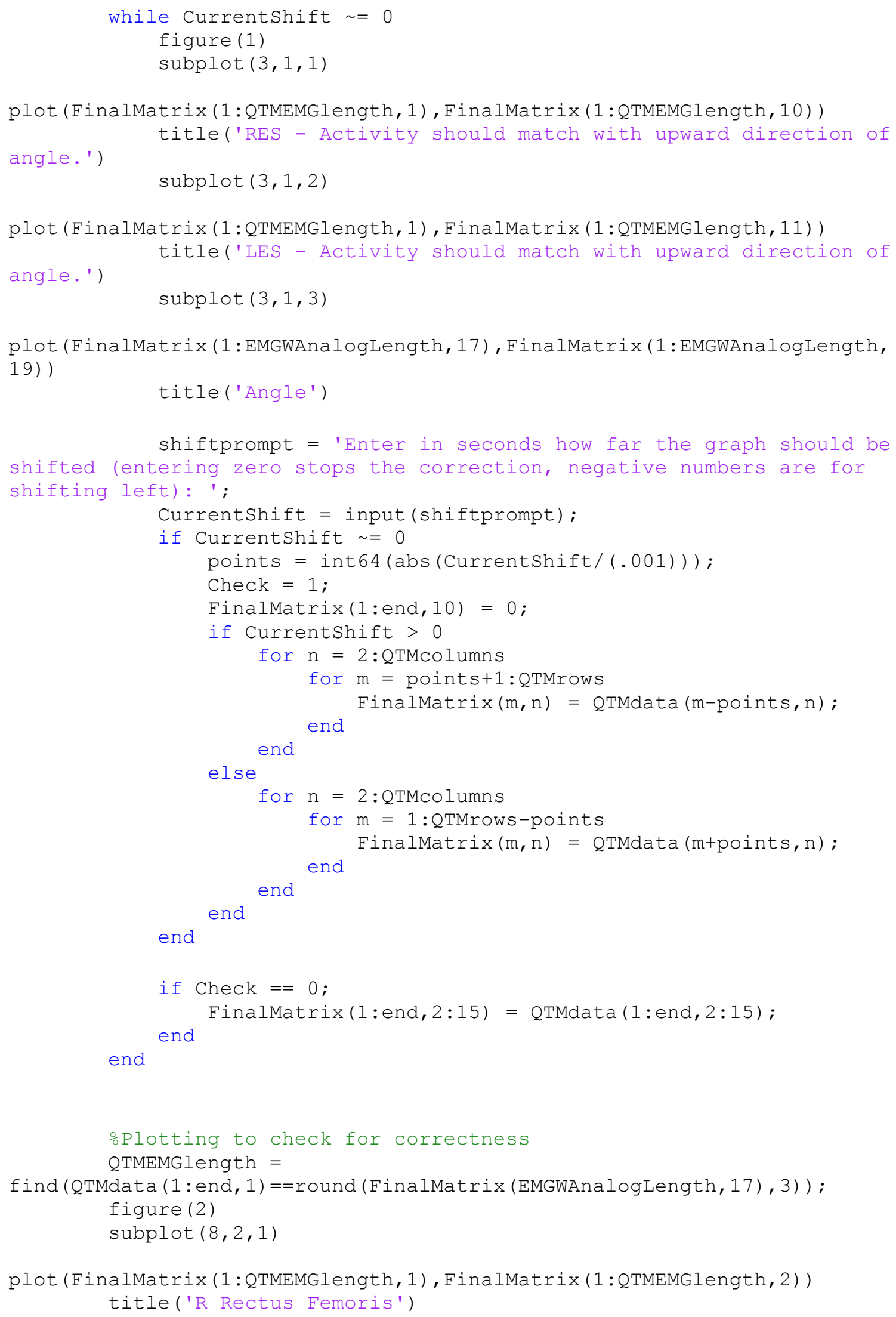




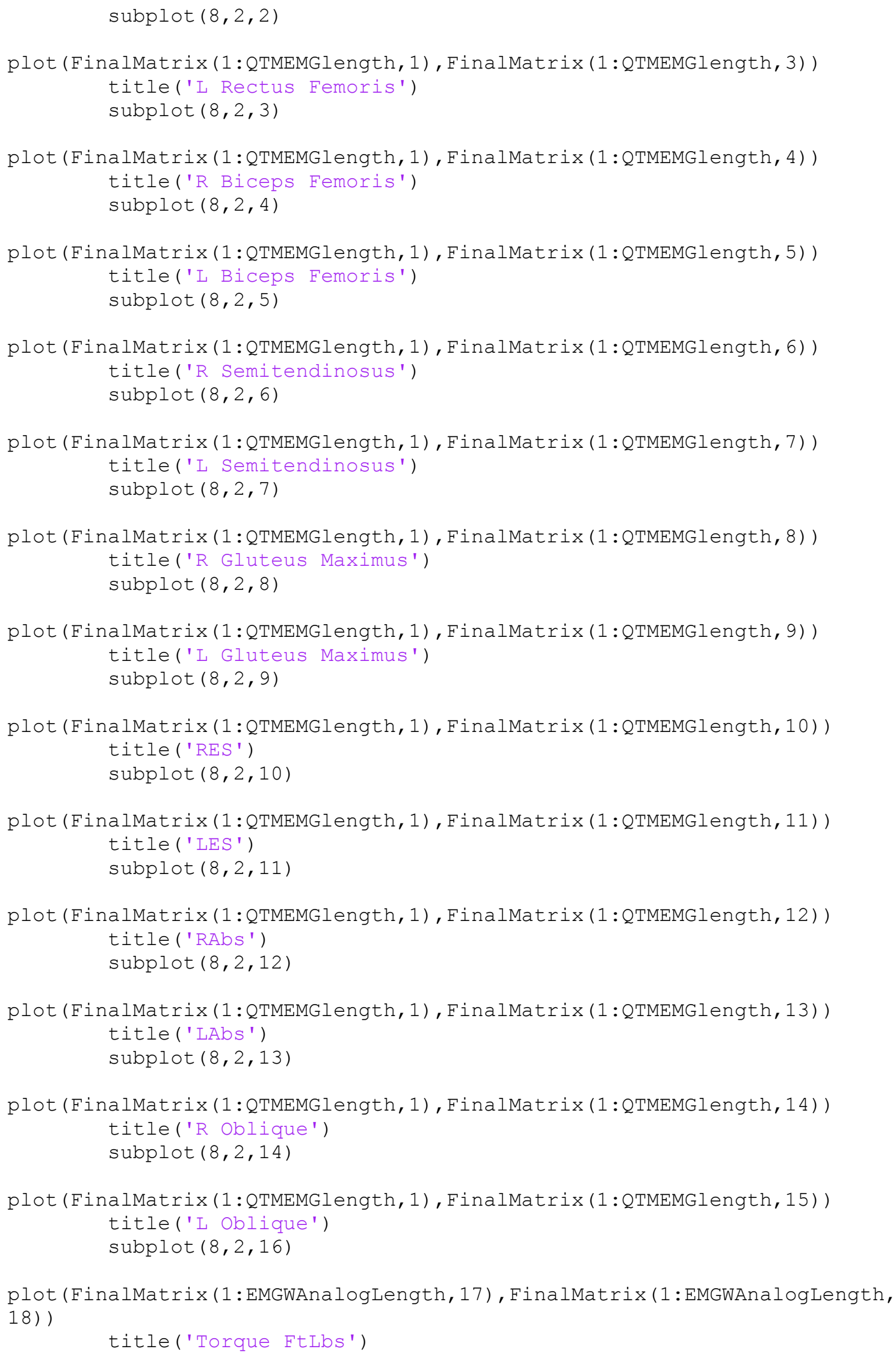




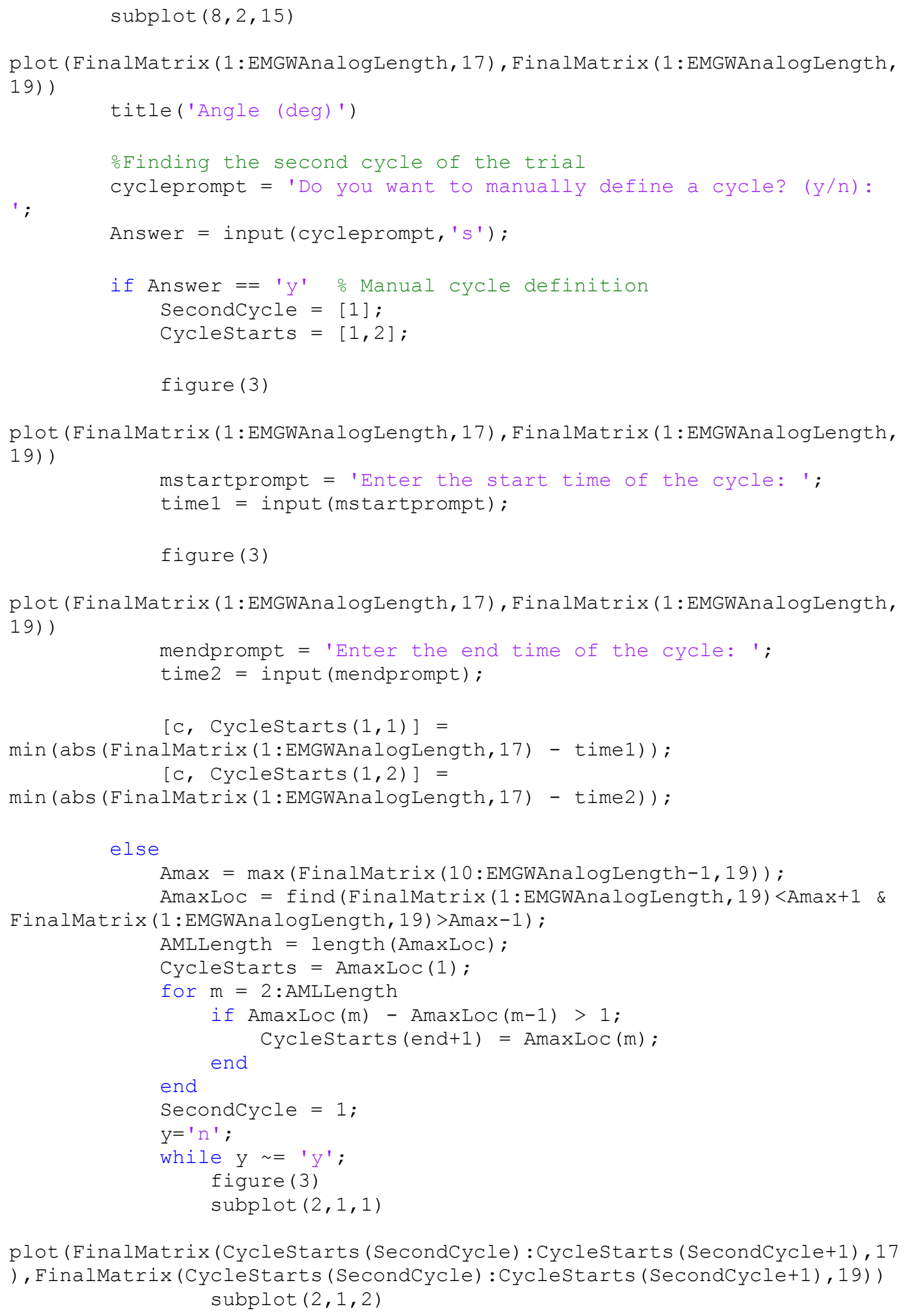


plot (FinalMatrix(1:EMGWAnalogLength, 17), FinalMatrix (1:EMGWAnalogLength, 19))

$(y / n) ? ' ;$ prompt $=$ 'Does the graph display the second cycle?

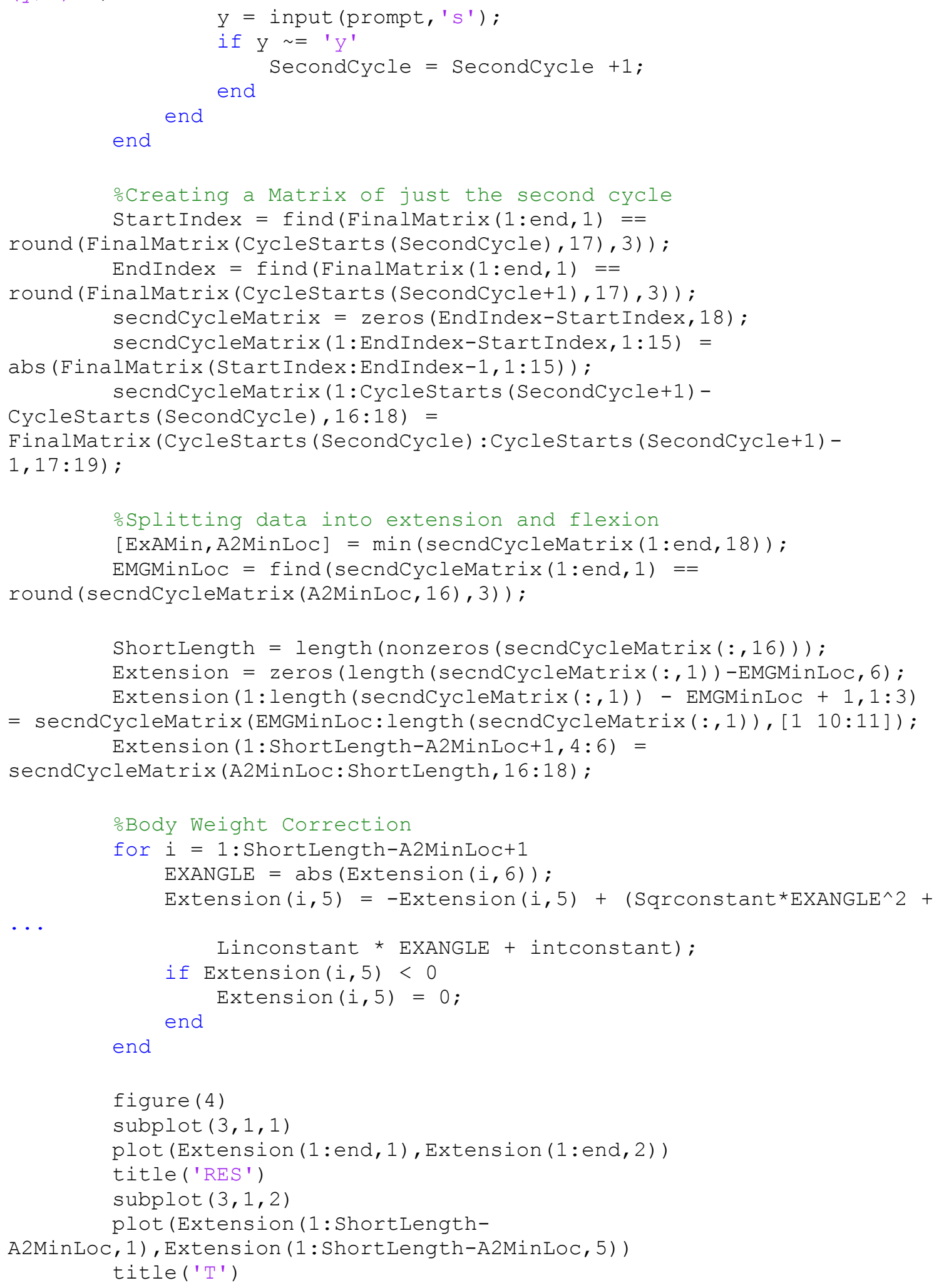




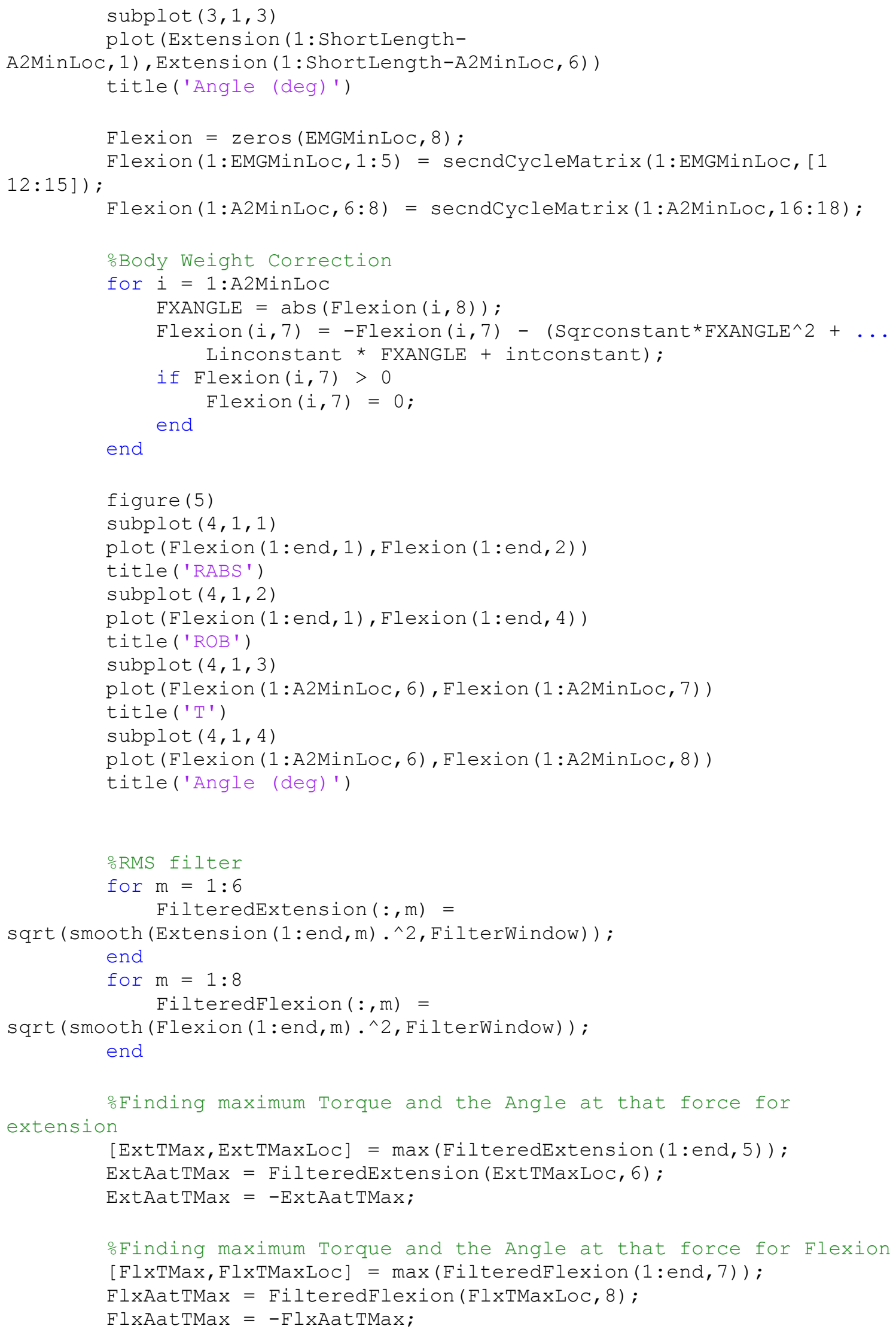




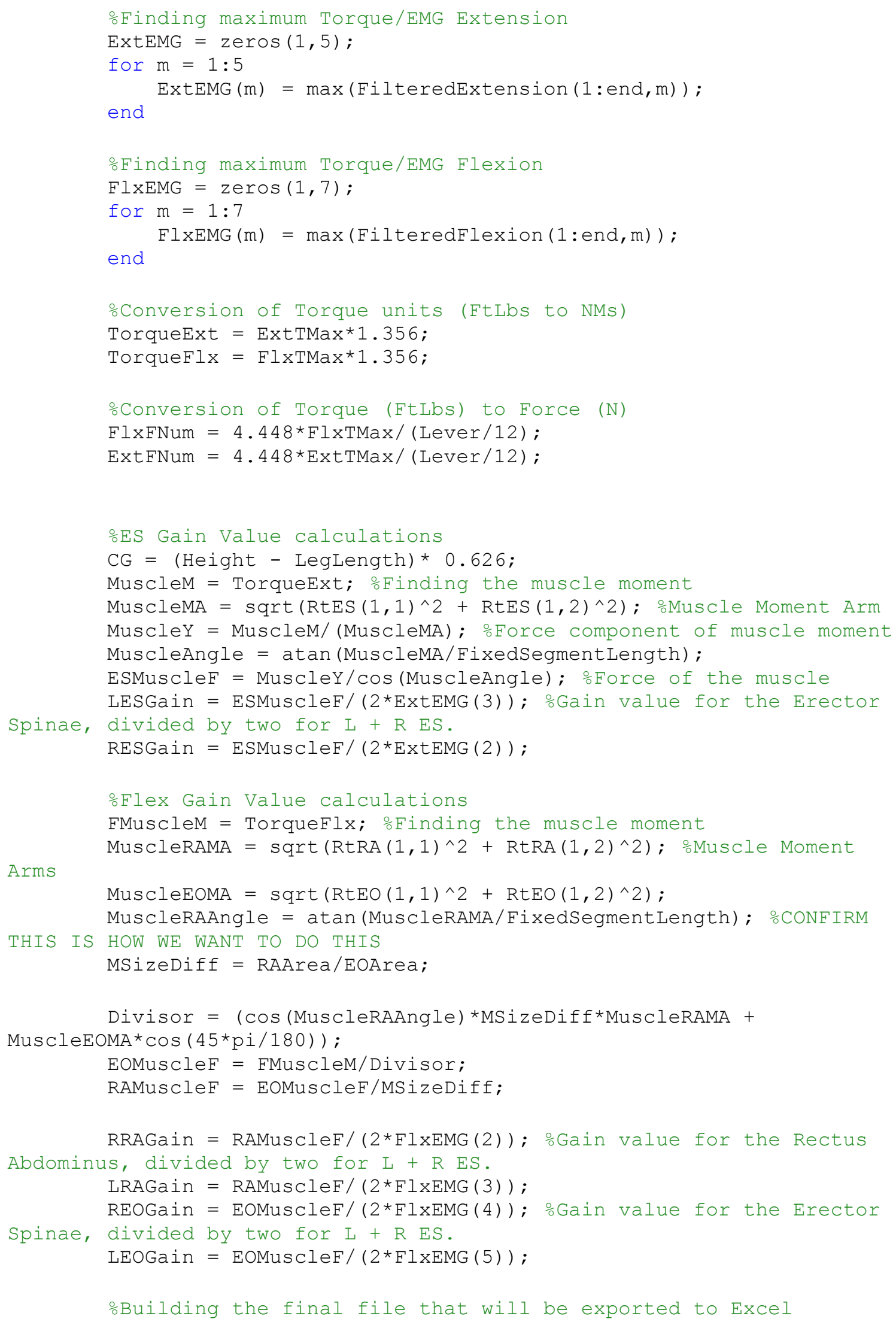




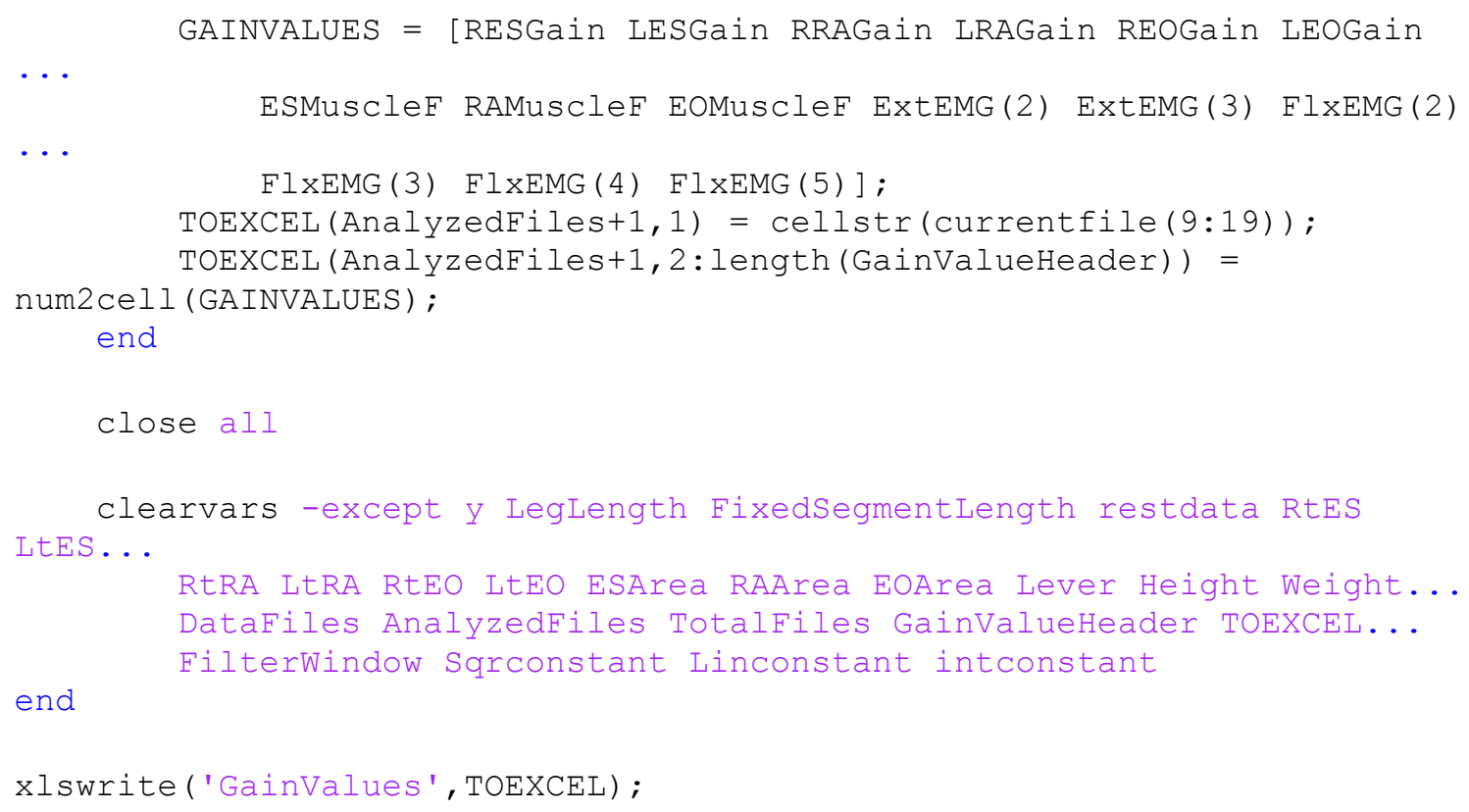




\section{APPENDIX C. VISUAL3D CODE}

File_New

;

File Open

$/$ FILE NAME $=$

! /SUFFIX $=$

!/SET_PROMPT=File_Open

;

Assign_Tags_To_Files

/MOTION_FILE_NAMES $=$ ALL_FILES

! /QUERY=

$/$ TAGS $=$ Trials

;

Set_Pipeline_Parameter_To_Folder_Path

/PARAMETER NAME=FOLDER

!/PARAMETER VALUE=VISUAL3D DEFAULT DATA FOLDER

! /PARAMETER VALUE MOTION_FILE $=$

! /PARAMETER VALUE SEARCH FOR $=$

!/PARAMETER_VALUE_REPLACE_WITH=

!/PARAMETER VALUE_APPEND=

!/SET_PROMPT=Select a directory

;

Create Hybrid Model

/CALIBRATION_FILE=::FOLDER\&*static00*.c3d

!/SUFFIX $=$

/RANGE $=$ ALL_FRAMES

;

Apply_Model_Template

!/MODEL_TEMPLATE $=$

! /CALIBRATION_FILE $=$

;

Assign_Model_File

! /CALIBRATION_FILE $=$

/MOTION_FILE_NAMES $=*$ *c3d

!/REMOVE_EXISTING_ASSIGNMENTS=FALSE 


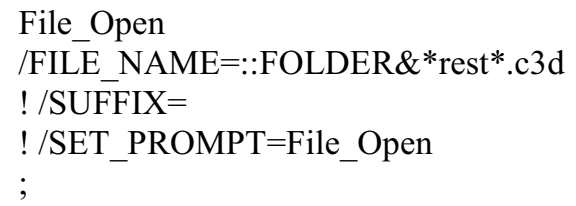

Assign_Tags_To_Files

/MOTION_FILE_NAMES $=*$ walk*

! $/ \mathrm{QUERY}=$

/TAGS=Normal Walk

;

Assign_Tags_To_Files

/MOTION_FILE_NAMES $=*$ sitstand $*$ ! $/ \mathrm{QUERY}=$

/TAGS $=$ Sit to Stand

;

Assign Tags To Files /MOTION_FILE_NAMES $=*$ stleg* ! /QUERY=

/TAGS=Straight Knee

;

Assign Tags To Files

/MOTION FILE NAMES $=*$ ballo*

! /QUERY=

/TAGS=Normal Pick Up ; 


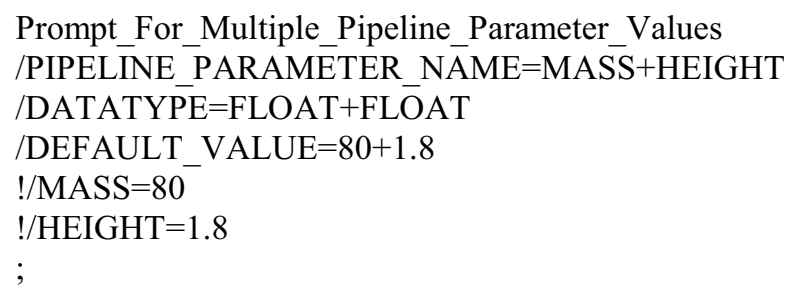

Select_Active_File /FILE_NAME=ALL_FILES

Set_Model_Metric

/CĀLIBRATION_FILE $=$ ALL_FILES

/METRIC_NAME $=$ Mass

/METRIC_VALUE=::MASS

;

Multiply_Signals_By_Constant /SIGNAL_TYPES=METRIC

/SIGNAL_FOLDER=PROCESSED

/SIGNAL_NAMES=MASS

/RESULT_TYPES=METRIC

/RESULT_FOLDER=PROCESSED

/RESULT_NAMES $=$ Torso_wt

!/RESULT_SUFFIX=

! /SIGNAL_COMPONENTS=

/CONSTANT $=0.67$ 
!Convert $\mathrm{kg}$ to $\mathrm{lbs} \times \mathrm{x} 67 \%$ of body weight

;

Set Model Metric

!/CÄLIBRATION_FILE=

/METRIC_NAME=Height

/METRIC_VALUE=::HEIGHT

;

Set_Pipeline_Parameter

/PARAMETER_NAME=EMG_SIGNALS

/PARAMETER_VALUE $=$ LAbs + LBicFem + LES + LGlutMax + LOblique + LRectFem + LSemiten +

RAbs +RBicFem + RES + RGlutMax + ROblique +RRectFem + RSemiten

! /PARAMETER_VALUE_SEARCH_FOR $=$

!/PARAMETER_VALUE_REPLACE_WITH=

!/PARAMETER_VALUE_PREFIX=

!/PARAMETER_VALUE_APPEND=

!/MULTI_PASS=FALSE

;

Select_Active_File

/FILE_NAME $=$ ALL_FILES

! /QUERY=

;

Interpolate

/SIGNAL_TYPES=TARGET

/SIGNAL_FOLDER=ORIGINAL

! /SIGNAL_NAMES=

/RESULT_FOLDER=PROCESSED

! /RESULT_SUFFIX $=$

! /MAXIMŪM_GAP $=10$

$! /$ NUM_FIT $=3$

! /POLYNOMIAL_ORDER $=3$

;

\author{
Highpass_Filter \\ /SIGNAL_TYPES $=$ ANALOG \\ /SIGNAL_FOLDER $=$ ORIGINAL \\ /SIGNAL_NAMES=::EMG_SIGNALS \\ /RESULT_FOLDER=PROCESSED \\ ! /RESULT_SUFFIX= \\ ! /FILTER_CLASS=BUTTERWORTH \\ /FREQUENCY_CUTOFF $=50.0$ \\ /NUM_REFLECTED $=0$ \\ ! /NUM_EXTRAPOLATED $=0$ \\ !/TOTĀL_BUFFER_SIZE $=100$
}




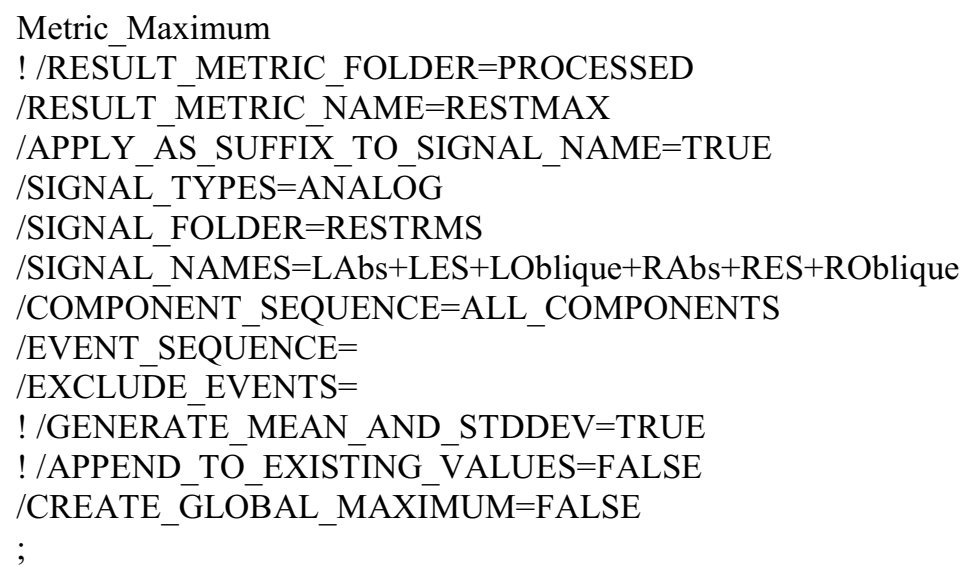




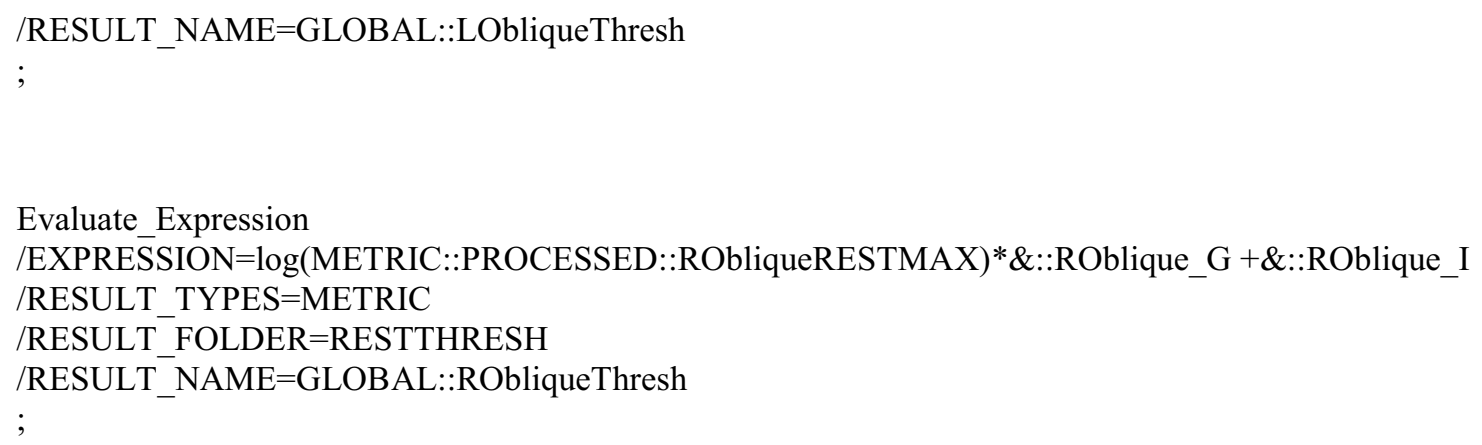


Evaluate_Expression

/EXPRESSION=(ANALOG::RMS::RES)*\&::RES_G+\&::RES_I

/RESULT TYPES=DERIVED

/RESULT ${ }^{-}$FOLDER=FORCES

/RESULT_NAME=RES

;

Evaluate Expression

/EXPRESSION=(ANALOG::RMS::LES)*\&::LES_G+\&::LES_I

/RESULT TYPES=DERIVED

/RESULT_FOLDER=FORCES

/RESULT_NAME $=$ LES

;

Evaluate Expression

/EXPRESSION=log(ANALOG::RMS::LAbs)*\&::LAbs_G+\&::LAbs_I

/RESULT TYPES=DERIVED

/RESULT FOLDER=FORCES

/RESULT_NAME=Labs

;

Evaluate_Expression

/EXPRESSION=log(ANALOG::RMS::RAbs)*\&::RAbs_G+\&::RAbs_I

/RESULT TYPES=DERIVED

/RESULT FOLDER $=$ FORCES

/RESULT_NAME=RAbs

Evaluate_Expression

/EXPRESSION= $\log ($ ANALOG::RMS::ROblique)*\&::ROblique_G+\&::ROblique_I

/RESULT_TYPES=DERIVED

/RESULT_FOLDER=FORCES

/RESULT_NAME=ROblique

;

Evaluate_Expression

/EXPRESSION= $\log$ (ANALOG::RMS::LOblique)*\&::LOblique_G+\&::LOblique_I

/RESULT TYPES=DERIVED

/RESULT_FOLDER=FORCES

/RESULT_NAME=LOblique

;

Set_Data_To_New_Values

/SIGNAL TY'PES=DERIVED

/SIGNAL_FOLDER=FORCES 
/SIGNAL NAMES=RES

!/SIGNA $\bar{L}$ COMPONENTS $=$ ALL COMPONENTS

/RESULT FOLDER=FORCESTHRESH

/RESULT SUFFIX=THRESH

!/EVENT_SEQUENCE=

! /EXCLUDE EVENTS=

/START_FRAME $=1$

/END_FRAME $=3000$

/USE_POINT_RATE=TRUE

/REPLACEMENT_VALUES $=0$

/THRESHOLD_HIGH=999999

/THRESHOLD_LOW=GLOBAL::METRIC::RESTTHRESH::RESThresh ;

Set Data To_New_Values

/SIGNAL TYPES=DERIVED

/SIGNAL_FOLDER=FORCES

/SIGNAL NAMES=LES

!/SIGNAL_COMPONENTS=ALL_COMPONENTS

/RESULT_FOLDER=FORCESTHRESH

/RESULT_SUFFIX=THRESH

!/EVENT_SEQUENCE=

!/EXCLUDE EVENTS=

/START_FRAME $=1$

/END_FRAME $=3000$

/USE_POINT_RATE $=$ TRUE

/REPLACEMENT_VALUES $=0$

/THRESHOLD HIGH=999999

/THRESHOLD_LOW= GLOBAL::METRIC::RESTTHRESH::LESThresh ;

Set_Data_To_New_Values

/SIGNAL_TYPES=DERIVED

/SIGNAL_FOLDER=FORCES

/SIGNAL ${ }^{-}$NAMES=RAbs

!/SIGNA $\bar{L}$ COMPONENTS=ALL_COMPONENTS

/RESULT_FOLDER=FORCESTHRESH

/RESULT_SUFFIX=THRESH

! /EVENT_SEQUENCE $=$

!/EXCLUDE_EVENTS=

/START_FRAME $=1$

/END_FRAME $=3000$

/USE POINT RATE $=$ TRUE

/REPLACEMENT_VALUES $=0$

/THRESHOLD HIGH=999999

/THRESHOLD_LOW=GLOBAL::METRIC::RESTTHRESH::RAbsThresh ;

Set_Data_To_New_Values

/SIGNAL_TY'PES=DERIVED

/SIGNAL_FOLDER=FORCES 
/SIGNAL NAMES=Labs

!/SIGNA $\bar{L}$ COMPONENTS $=$ ALL COMPONENTS

/RESULT FOLDER=FORCESTHRESH

/RESULT SUFFIX=THRESH

!/EVENT_SEQUENCE=

! /EXCLUDE EVENTS=

/START_FRAME $=1$

/END_FRAME $=3000$

/USE_POINT_RATE=TRUE

/REPLACEMENT_VALUES $=0$

/THRESHOLD_HIGH=999999

/THRESHOLD_LOW=GLOBAL::METRIC::RESTTHRESH::LabsThresh

;

Set Data To_New_Values

/SIG̈NAL TY'PES=-DERIVED

/SIGNAL_FOLDER=FORCES

/SIGNAL ${ }^{-}$NAMES $=$LOblique

!/SIGNAL_COMPONENTS=ALL_COMPONENTS

/RESULT_FOLDER=FORCESTHRESH

/RESULT_SUFFIX=THRESH

!/EVENT_SEQUENCE=

!/EXCLUDE EVENTS=

/START_FRAME $=1$

/END_FRAME $=3000$

/USE_POINT_RATE $=$ TRUE

$/$ REPLACEMENT_VALUES $=0$

/THRESHOLD_HIGH=999999

/THRESHOLD_LOW= GLOBAL::METRIC::RESTTHRESH::LObliqueThresh ;

Set_Data_To_New_Values

/SIGNAL_TYPES=DERIVED

/SIGNAL_FOLDER=FORCES

/SIGNAL_NAMES=ROblique

/SIGNAL_COMPONENTS=ALL COMPONENTS

/RESULT_FOLDER=FORCESTHRESH

/RESULT_SUFFIX=THRESH

!/EVENT_SEQUENCE=

!/EXCLUDE_EVENTS=

/START_FRAME $=1$

/END_FRAME $=3000$

/USE POINT RATE $=$ TRUE

/REPLACEMENT_VALUES $=0$

/THRESHOLD HIGH=999999

/THRESHOLD_LOW= GLOBAL::METRIC::RESTTHRESH::RObliqueThresh ;

Set_Data_To_New_Values

/SIGNAL_TY'PES=DERIVED

/SIGNAL_FOLDER=FORCESTHRESH 
/SIGNAL NAMES $=$ RESTHRESH

! /SIGNAL COMPONENTS $=$ ALL COMPONENTS

/RESULT_FOLDER=FORCES

$/$ RESULT SUFFIX $=$ Final

!/EVENT_SEQUENCE=

!/EXCLUDE_EVENTS $=$

!/START_FRAME=

! /END FRAME $=$

/USE_POINT_RATE $=$ TRUE

/REPLACEMENT VALUES $=0$

$! /$ THRESHOLD_HIGH=NO_DATA

/THRESHOLD_LOW $=0$

;

Set Data To_New_Values

/SIGNAL TYPES=-DERIVED

/SIGNAL_FOLDER=FORCESTHRESH

/SIGNAL_NAMES=LESTHRESH

! /SIGNAL_COMPONENTS=ALL_COMPONENTS

/RESULT_FOLDER=FORCES

/RESULT_SUFFIX $=$ Final

!/EVENT_SEQUENCE=

! /EXCLUDE EVENTS=

!/START_FRAME $=$

!/END_FRAME $=$

!/USE_POINT_RATE=TRUE

/REPLACEMENT - VALUES $=0$

! /THRESHOLD_HIGH=NO_DATA

/THRESHOLD_LOW $=0$ ;

Set Data_To_New_Values

/SIGNAL_TY'PES=DERIVED

/SIGNAL_FOLDER=FORCESTHRESH

/SIGNAL ${ }^{-}$NAMES=LAbsTHRESH

! /SIGNA $\bar{L}$ COMPONENTS=ALL COMPONENTS

/RESULT_FOLDER=FORCES

/RESULT_SUFFIX=Final

! /EVENT_SEQUENCE=

! /EXCLUDE_EVENTS=

! /START_FRAME=

! /END_FRAME $=$

!/USE_POINT_RATE=TRUE

/REPLACEMENT_VALUES $=0$

! /THRESHOLD HIGH=NO DATA

/THRESHOLD_LOW $=0$

;

Set_Data_To_New_Values

/SIGNAL_TY'PES=DERIVED

/SIGNAL_FOLDER=FORCESTHRESH 
/SIGNAL NAMES=RAbsTHRESH

! /SIGNAL COMPONENTS=ALL COMPONENTS

/RESULT_FOLDER=FORCES

$/$ RESULT SUFFIX $^{-}$Final

!/EVENT_SEQUENCE=

! /EXCLUDE EVENTS=

!/START_FRAME=

!/END_FRAME $=$

!/USE_POINT_RATE $=$ TRUE

/REPLACEMENT VALUES $=0$

$! /$ THRESHOLD_HIGH=NO_DATA

/THRESHOLD_LOW $=0$

;

Set_Data_To_New_Values

/SIGNAL TYPES=-DERIVED

/SIGNAL_FOLDER=FORCESTHRESH

/SIGNAL_NAMES=RObliqueTHRESH

! /SIGNAL_COMPONENTS=ALL_COMPONENTS

$/$ RESULT_FOLDER=FORCES

/RESULT_SUFFIX=Final

! /EVENT_SEQUENCE $=$

! /EXCLUDE EVENTS=

!/START_FRAME $=$

!/END_FRAME $=$

!/USE_POINT_RATE=TRUE

/REPLACEMENT_VALUES $=0$

! /THRESHOLD_HIGH=NO_DATA

/THRESHOLD_LOW $=0$ ;

Set Data_To_New_Values

/SIGNAL_TY'PES=DERIVED

/SIGNAL_FOLDER=FORCESTHRESH

/SIGNAL NAMES=LObliqueTHRESH

! /SIGNAL__COMPONENTS=ALL_COMPONENTS

/RESULT_FOLDER=FORCES

/RESULT_SUFFIX=Final

!/EVENT_SEQUENCE=

! /EXCLUDE_EVENTS=

! /START_FRAME=

!/END_FRAME $=$

!/USE_POINT_RATE $=$ TRUE

/REPLACEMENT_VALUES $=0$

! /THRESHOLD_HIGH $=$ NO_DATA

/THRESHOLD_LOW $=0$

;

Compute_Planar_Angle

/SIGNAL_TYPES=TARGET

/SIGNAL_FOLDER=PROCESSED 
/SIGNAL_NAMES $=$ T12+L2+L4+SACR

! /RESULT $\bar{T}$ FOLDER=PROCESSED

/RESULT NAME=LUMBAR_ANGLE

/COMPUTE_3PT_ANGLE=FĀLSE

! $/$ NORMALX $=$

! $/$ NORMALY=

! $/$ NORMALZ $=$

!/REFERENCE SEGMENT $=$ LAB

/PROJECTION_PLANE $=$ YZ

! /USE RIGHT HAND RULE $=$ TRUE

/USE_0_TO_360_DEGREES=FALSE

;

Compute_Model_Based_Data

/RESULT_NAME $=$ TORSO_ANGLE

/FUNCTIŌN=JOINT ANGLE

$/ \mathrm{SEGMENT}=\mathrm{L} 2 \mathrm{~L} 4$

/REFERENCE_SEGMENT=Virtual Lab

/RESOLUTION_COORDINATE_SYSTEM=

!/USE_CARDAN_SEQUENCE=FALSE

!/NORMALIZATION=FALSE

! /NORMALIZATION METHOD=

!/NORMALIZATION METRIC $=$

NEGATEX=TRUE

! $/$ NEGATEY $=$ FALSE

! $/$ NEGATEZ $=$ FALSE

! $/ \mathrm{AXIS} 1=\mathrm{X}$

! $/$ AXIS2 $=Y$

! $/ \mathrm{AXIS} 3=\mathrm{Z}$

;

Evaluate_Expression

/EXPRESSION=METRIC::PROCESSED::Torso_wt::X

*cos(LINK_MODEL_BASED::ORIGINAL::TORSO_ANGLE::X*pi()/180)*9.8

/RESULT TYPES=DERIVED

/RESULT ${ }^{-}$FOLDER $=$FORCES

/RESULT_NAME=Torso

;

Evaluate_Expression

/EXPRESSION=METRIC::PROCESSED::Torso_wt::X

*sin(LINK_MODEL_BASED::ORIGINAL::TORSO_ANGLE::X*pi()/180)*9.8

/RESULT TYPES $=\overline{\text { DERIVED }}$

/RESULT FOLDER=FORCES

/RESULT_NAME=Torso_Shear ;

Evaluate_Expression

/EXPRESSION=DERIVED::FORCES::RObliqueTHRESHFinal*sqrt(2)/2 


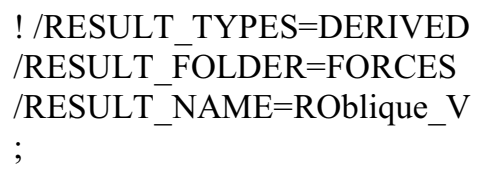

Export_Data_To_Ascii_File /SIGNĀL_TY'PES=DERIVED+DERIVED+DERIVED+DERIVED+DERIVED+DERIVED+DERIVED+ 
DERIVED+ANALOG+ANALOG +ANALOG+ANALOG+ANALOG+ANALOG

/SIGNAL FOLDER=PROCESSED+PROCESSED+FORCES+FORCES+FORCES+FORCES+FORCES+ FORCES+ORIGINAL+ORIGINAL+ORIGINAL+ORIGINAL+ORIGINAL+ORIGINAL

/SIGNAL_NAMES=Load+Shear+LAbs+LES+LOblique+RAbs+RES+ROblique+LAbs+LES+LOblique+ RAbs+RES + ROblique

! /FILE NAME $=$

! /SIGNAL COMPONENTS $=$

/COMPONENT_SEQUENCE=ALL

/SIGNAL PREC $\bar{C} I S I O N=5$

! /START_LABEL $=$

! /END_LABEL=

!/EVENT_SEQUENCE=

! /EXCLUDE EVENTS=

!/USE_POINT_RATE $=$ FALSE

! /NORMALIZ $\bar{E}$ DATA=FALSE

! /NORMALIZE POINTS $=101$

!/EXPORT_MEAN_AND_STD_DEV=FALSE

!/USE_P2D_FORMAT $=$ FALSE

!/USE_XML_FORMAT=FALSE

! /USE_SHORT_FILENAME=FALSE

! /EXPORT EMPTY SIGNALS=FALSE

!/EXPORT_WITHOUTT_HEADER=FALSE

!/EXPORT_NAN=FALSE

; 


\section{APPENDIX D. STATISTICAL RESULTS}

Table D-1. Full Multiple Measure ANOVA Results for the REO.

\begin{tabular}{llcccccc}
\hline \multicolumn{1}{c}{ Effect } & & & & & \multicolumn{2}{c}{ Partial eta } \\
squared
\end{tabular}

An asterisk $\left(^{*}\right)$ indicates that an interaction effect between the two or more variables had an effect on the percent error 
Table D-2. Full Multiple Measure ANOVA Results for the LEO.

\begin{tabular}{lccccccc}
\hline \multicolumn{1}{c}{ Effect } & Method & Value & F & Hypothesis df & Error df & Sig. & Partial eta \\
squared
\end{tabular}

An asterisk $(*)$ indicates that an interaction effect between the two or more variables had an effect on the percent error 
Table D-3. Full Multiple Measure ANOVA Results for the RRA.

\begin{tabular}{lccccccc}
\hline \multicolumn{1}{c}{ Effect } & Method & Value & F & Hypothesis df & Error df & Sig. & Partial eta \\
squared
\end{tabular}

An asterisk $(*)$ indicates that an interaction effect between the two or more variables had an effect on the percent error 
Table D-4. Full Multiple Measure ANOVA Results for the LRA.

\begin{tabular}{lccccccc}
\hline \multicolumn{1}{c}{ Effect } & & & & & & \multicolumn{2}{c}{ Partial eta } \\
squared
\end{tabular}

An asterisk $\left(^{*}\right)$ indicates that an interaction effect between the two or more variables had an effect on the percent error 
Table D-5. Full Multiple Measure ANOVA Results for the RES.

\begin{tabular}{lccccccc}
\hline \multicolumn{1}{c}{ Effect } & Method & Value & F & Hypothesis df & Error df & Sig. & Partial eta \\
squared
\end{tabular}

An asterisk $(*)$ indicates that an interaction effect between the two or more variables had an effect on the percent error 
Table D-6. Full Multiple Measure ANOVA Results for the LES.

\begin{tabular}{lccccccc}
\hline \multicolumn{1}{c}{ Effect } & & & & & \multicolumn{2}{c}{ Partial eta } \\
squared
\end{tabular}

An asterisk $\left(^{*}\right)$ indicates that an interaction effect between the two or more variables had an effect on the percent error 


\section{VITA}

Pablo Joaquin Dopico was born in Worcester, Massachusetts in 1991. He received his B.S. in Biomedical Engineering at Vanderbilt University in Nashville, TN in May 2013. One year later Pablo was accepted and entered into the department of Biomedical Engineering and Orthopedics at the University of Tennessee Health Science Center. With Dr. Mihalko as his advisor, Pablo developed an Electromyography based model for estimating L4/L5 intervertebral disk loading. His research has been presented at Orthopedic Research Society, Gait and Clinical Movement Assessment Society, and Graduate Research Day, where he won a Best Poster award. In December 2016 Pablo received his Master's Degree for his research and in August 2016 he began working on his Doctor of Philosophy at the University of Florida in Gainesville, Florida. 